\title{
The Involvement of Rhopr-CRF/DH in Feeding and Reproduction in the Blood-gorging Insect Rhodnius prolixus
}

by

Shirin Mollayeva

A thesis submitted in conformity with the requirements for the degree of Master of Science

Cell \& Systems Biology

University of Toronto

(c) Copyright by Shirin Mollayeva 2017 


\title{
The Involvement of Rhopr-CRF/DH in Feeding and Reproduction in the Blood-gorging Insect Rhodnius prolixus
}

\author{
Shirin Mollayeva \\ Master of Science \\ Cell \& Systems Biology \\ University of Toronto
}

2017

\section{Abstract}

Rhodnius prolixus is a blood-gorging insect and vector of human Chagas disease, which is transmitted following feeding, when the insect excretes urine contaminated with the Trypanosoma cruzi parasite. Diuretic corticotropin-releasing factor-like peptide (Rhopr$\mathrm{CRF} / \mathrm{DH}$ ) plays a major role in this post-prandial diuresis; however, its localization in the insect's central nervous system and expression of its receptor in feeding-related tissues as well as the female reproductive system suggests a multifaceted role for the hormone, beyond diuresis. Here, the involvement of Rhopr-CRF/DH in feeding and reproduction was investigated. Rhopr$\mathrm{CRF} / \mathrm{DH}$-like staining decreased in neurosecretory cells of the CNS after feeding, and injection of the hormone resulted in intake of a significantly smaller blood meal. Adult females injected with Rhopr-CRF/DH produced and laid significantly fewer eggs, and application of Rhopr$\mathrm{CRF} / \mathrm{DH}$ to the lateral oviducts inhibited contractions. These findings have implications for vector control by highlighting targets to alter feeding, diuresis, and reproduction of this disease vector. 


\section{Acknowledgments}

I am immensely grateful for the past two years that I've been part of the Lange Lab. I owe most of my gratitude to my supervisor, Prof. Angela Lange, who took me on when no one else would and provided me with the guidance and support to grow in a science completely new to me, and which I have come to love as a result, thank you Angela. I am also grateful to Prof. Ian Orchard, essentially my co-supervisor, for providing me with direction in my experiments, for reading my work, giving feedback, for caring, thank you. I would also like to thank Prof. Joel Levine for being in my committee, for his feedback and direction, and Prof. Ted Erclik for agreeing to act as my external examiner, your time is greatly appreciated.

Thank you to some of the best people I have ever met, my lab mates, past and present: Amir, Marina, Laura, Sara, Hussain, Thiago, Sam, Rachel, Douglas, and Merima. Thank you for your time and patience training me, and for your unwavering willingness to listen and offer advice on anything from the science to the personal. Thank you for the laughs, the memories we have made will be cherished. I wish you all happiness and success, and hope we make time to see each other even as we move forward in new pursuits.

Last but not least, a huge thank you to my family, for encouraging me to pursue things I would otherwise avoid out of fear of failure. Thank you for bringing me up when I was down and pushing me to move forward, to be persistent, to not give up. I feel so lucky to have you. 


\section{Thesis Organization}

This thesis is organized into chapters, with the first and last chapters comprising expanded introduction and discussion sections, respectively. The introductory chapter features a review of CRF signaling and its effects in vertebrates and invertebrates, including the model organism in this study, Chagas disease vector Rhodnius prolixus. Chapter 2 is a manuscript currently in press (Mollayeva S, Orchard I, Lange AB. The involvement of Rhopr-CRF/DH in feeding and reproduction in the blood-gorging insect Rhodnius prolixus. Gen. Comp. Endocrinol. (2017), http://dx.doi.org/10.1016/j.ygcen.2017.07.005). This manuscript describes findings from experiments looking at the effect of increasing haemolymph Rhopr-CRF/DH titres on feeding and reproduction, and the effect of Rhopr-CRF/DH in in vitro oviduct contraction assays. Chapter 3 features additional experiments not described in the manuscript, in which dsRNA was synthesized and injected into insects with the goal of knocking down Rhopr-CRF/DH and observing the effects in the aforementioned assays. Finally, the discussion reviews the findings in the context of the existing literature on the roles of CRF, particularly in insects. 


\section{Table of Contents}

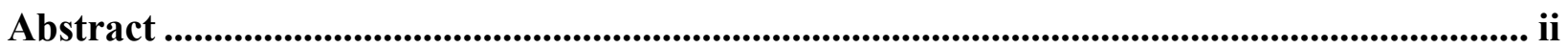

Acknowledgments ...................................................................................................................... iii

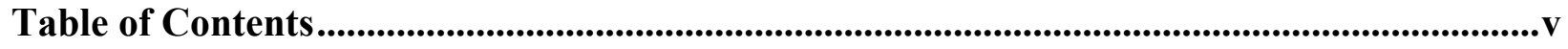

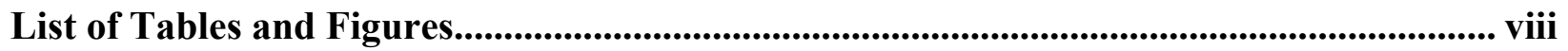

Chapter 1 General Introduction .......................................................................................................1

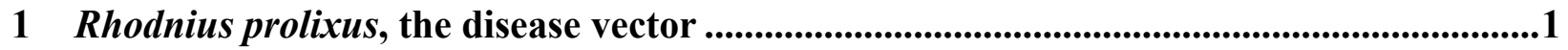

2 Corticotropin-releasing factor (CRF), its pathway, and modes of action in the

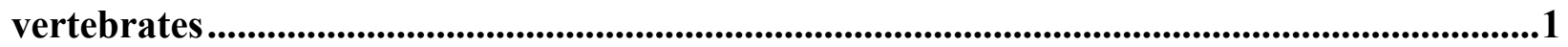

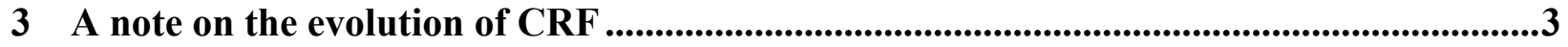

4 The link between feeding, reproduction, and CRF in vertebrates .................................4

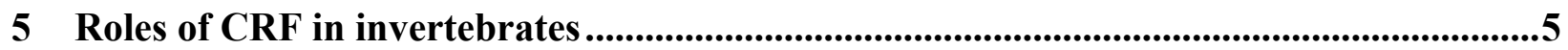

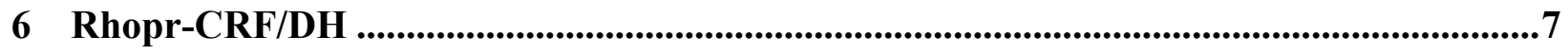

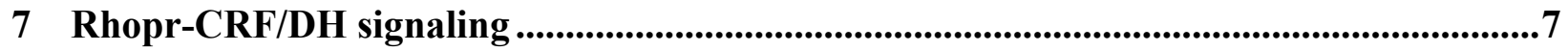

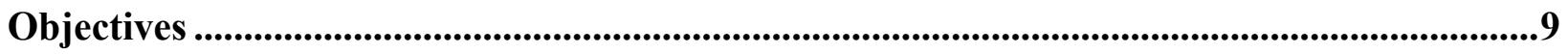

Chapter 2 The involvement of Rhopr-CRF/DH in feeding and reproduction in the bloodgorging insect Rhodnius prolixus (in press, Gen Comp Endocrinol 2017; doi:

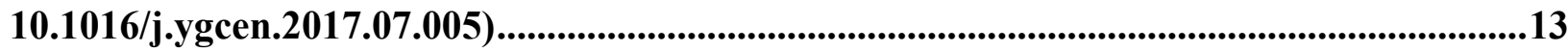

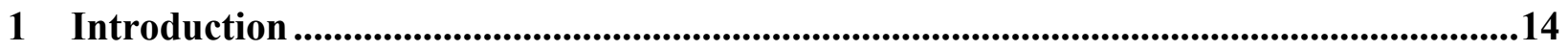

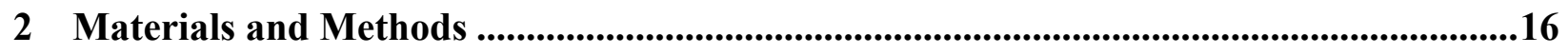

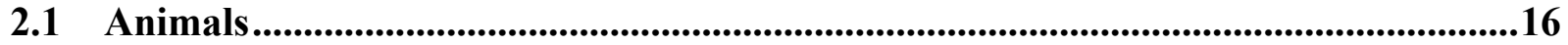

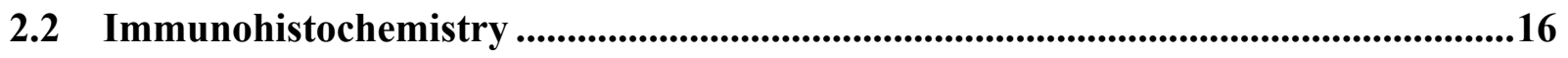

2.3 Feeding assay ..........................................................................................................17

2.4 Egg-laying assay .............................................................................................................17

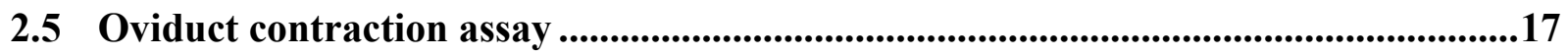

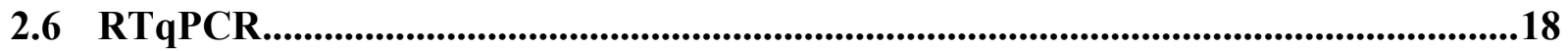

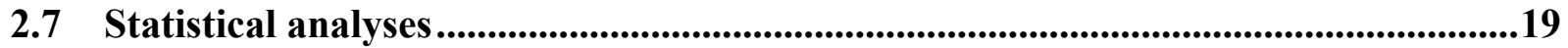




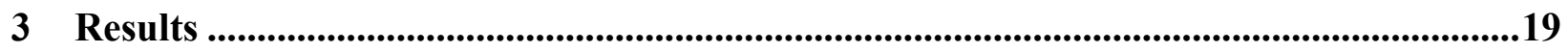

3.1 CRF/DH-like immunostaining in the CNS.........................................................19

3.2 CRF-like immunostaining in the CNS after feeding ......................................................24

3.3 qPCR: expression of Rhopr-CRF/DH transcript in the CNS after feeding...............24

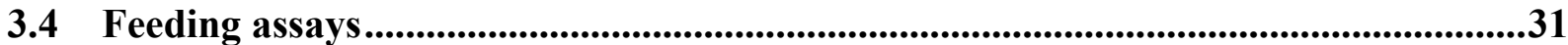

3.5 Egg-laying assays .......................................................................................................40

3.6 Contraction assays...................................................................................................................40

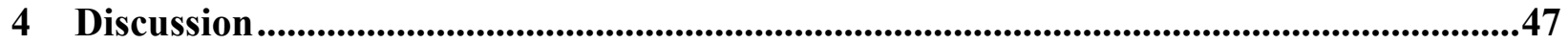

Chapter 3 RNAi: Silencing Rhopr-CRF/DH and its receptor......................................................54

1 Introduction ...........................................................................................................................................54

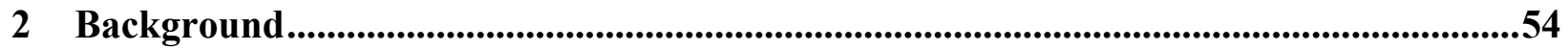

2.1 RNA interference: dsRNA processing...........................................................................54

2.2 dsRNA uptake and impact....................................................................................................55

2.3 Methods of dsRNA delivery...............................................................................................57

2.4 RNAi in insects: vector control considerations...............................................................60

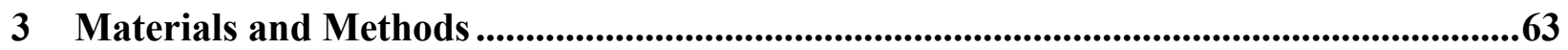

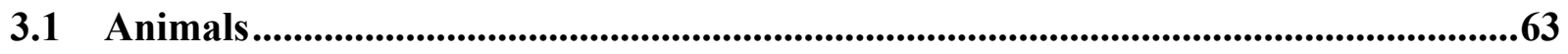

3.2 Double-stranded RNA (dsRNA) synthesis .....................................................................63

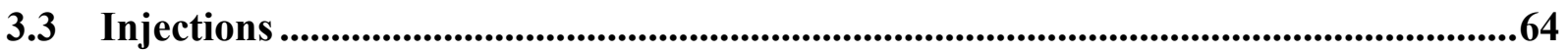

3.4 qPCR to determine knockdown efficiency ......................................................................65

3.5 Immunohistochemistry ……............................................................................................66

3.6 Feeding assay .............................................................................................................66

3.7 Statistical analyses .............................................................................................................66

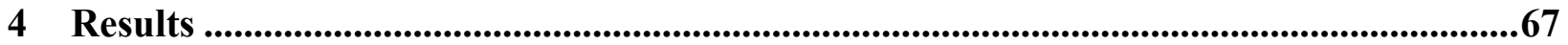

4.1 Rhopr-CRF/DH transcript knockdown efficiency and effects......................................67

4.2 Rhopr-CRF/DH-R2B transcript knockdown .....................................................................76

5 Discussion ......................................................................................................................

Chapter 4 General Discussion ...................................................................................................................84

1 Rhopr-CRF/DH and feeding .........................................................................................................84 
2 Rhopr-CRF/DH and reproduction ................................................................................86

3 Rhopr-CRF/DH and the stress response in insects ...................................................8

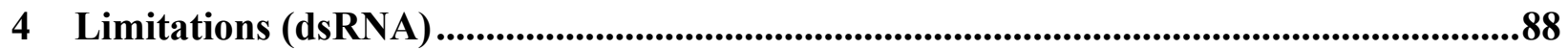

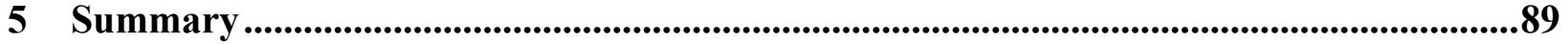

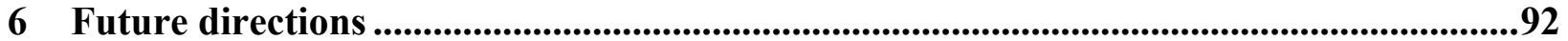




\section{List of Tables and Figures}

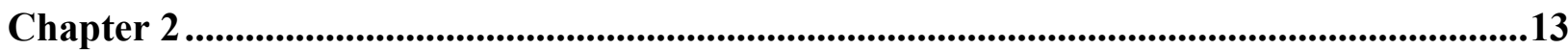

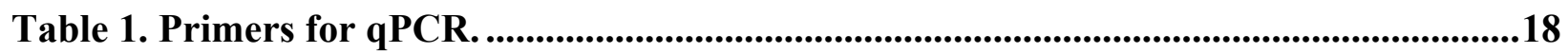

Figure 1. CRF-like immunostaining of $5^{\text {th }}$ instar and adult $R$. prolixus CNS.......................20

Figure 2. Composite maps of cells that stained consistently for CRF-like immunoreactivity in the CNS of $5^{\text {th }}$ instars and adults .................................................................................22

Figure 3. Time-course of CRF-like immunostaining in $5^{\text {th }}$ instar $R$. prolixus.........................25

Figure 4. Time-course of CRF-like immunostaining in $5^{\text {th }}$ instar $R$. prolixus before feeding, just after feeding and $2 \mathrm{~h}$ after feeding..........................................................................27

Figure 5. Temporal expression of the Rhopr-CRF/DH transcript in the brain and MTGM

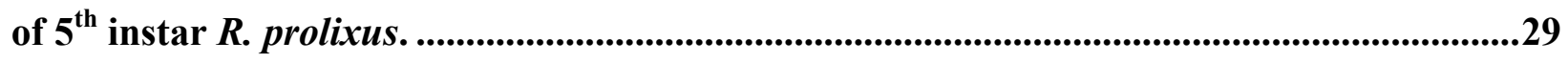

Figure 6. Feeding assay performed on $5^{\text {th }}$ instar $R$. prolixus. ...........................................32

Figure 7. Feeding assay performed on adult $R$. prolixus....................................................34

Figure 8. Weights of saline- and Rhopr-CRF/DH-injected insects over the course of 4 hours

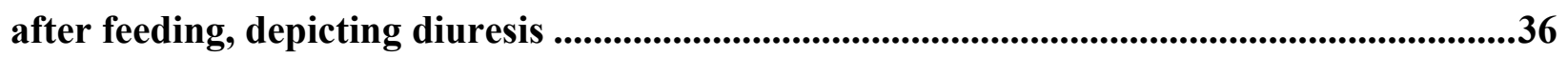

Figure 9. Feeding assay performed on $5^{\text {th }}$ instar $R$. prolixus. ..................................................38

Figure 10. Results of egg-laying assay ............................................................................41

Figure 11. Egg-laying assay cumulative results. ...............................................................................43

Figure 12. Results of in vitro oviduct contraction assay. ...........................................................45

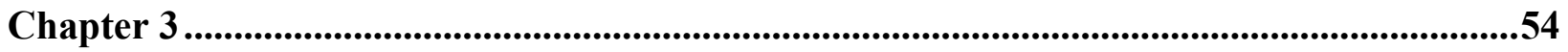

Table 1. Primers used for synthesis of dsRNA............................................................64

Table 2. Primers for qPCR. ......................................................................................................65

Figure 1. Results of qPCR analysis showing the relative expression of the Rhopr-CRF/DH transcript in the CNS of $5^{\text {th }}$ instar $R$. prolixus injected with dsRNA .....................................68

Figure 2. Results of immunohistochemistry showing Rhopr-CRF/DH-like immunostaining in the CNS of $5^{\text {th }}$ instar $R$. prolixus injected with dsRNA 
Figure 3. Feeding assay following dsRNA injection. ..............................................................72

Figure 4. Feeding assay following injection of antiserum.....................................................74

Figure 5. Results of qPCR analysis, showing the relative expression of the Rhopr-CRF/DHR2B transcript in the CNS of $5^{\text {th }}$ instar $R$. prolixus injected with dsCRFR and dsARG ......77

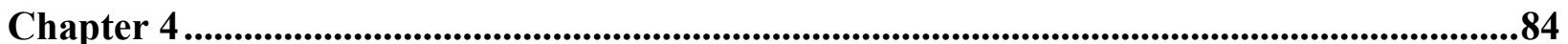

Figure 1. A schematic depicting the known and hypothesized mechanisms underlying the behavioural effects of Rhopr-CRF/DH localized in NSCs in the brain and MTGM. ...........90 


\section{List of Tables and Figures}

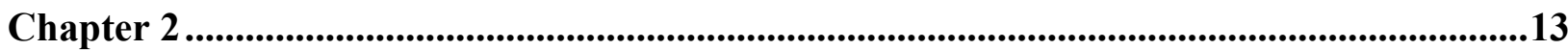

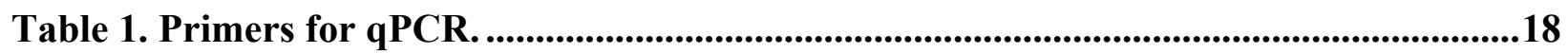

Figure 1. CRF-like immunostaining of $5^{\text {th }}$ instar and adult $R$. prolixus CNS.......................20

Figure 2. Composite maps of cells that stained consistently for CRF-like immunoreactivity in the CNS of $5^{\text {th }}$ instars and adults .................................................................................22

Figure 3. Time-course of CRF-like immunostaining in $5^{\text {th }}$ instar $R$. prolixus.........................25

Figure 4. Time-course of CRF-like immunostaining in $5^{\text {th }}$ instar $R$. prolixus before feeding, just after feeding and $2 \mathrm{~h}$ after feeding..........................................................................27

Figure 5. Temporal expression of the Rhopr-CRF/DH transcript in the brain and MTGM

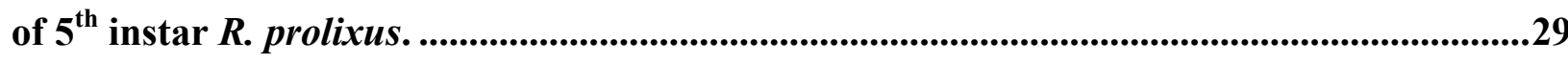

Figure 6. Feeding assay performed on $5^{\text {th }}$ instar $R$. prolixus. ...........................................32

Figure 7. Feeding assay performed on adult $R$. prolixus....................................................34

Figure 8. Weights of saline- and Rhopr-CRF/DH-injected insects over the course of 4 hours

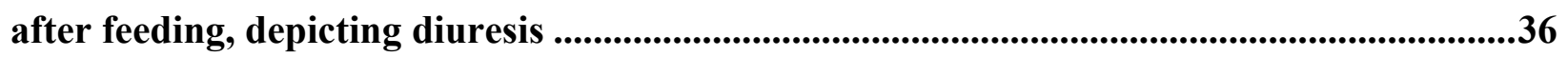

Figure 9. Feeding assay performed on $5^{\text {th }}$ instar $R$. prolixus. ..................................................38

Figure 10. Results of egg-laying assay ............................................................................41

Figure 11. Egg-laying assay cumulative results. ...............................................................................43

Figure 12. Results of in vitro oviduct contraction assay. ...........................................................45

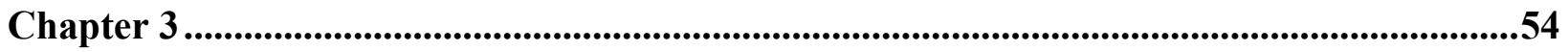

Table 1. Primers used for synthesis of dsRNA............................................................64

Table 2. Primers for qPCR. ......................................................................................................65

Figure 1. Results of qPCR analysis showing the relative expression of the Rhopr-CRF/DH transcript in the CNS of $5^{\text {th }}$ instar $R$. prolixus injected with dsRNA .....................................68

Figure 2. Results of immunohistochemistry showing Rhopr-CRF/DH-like immunostaining in the CNS of $5^{\text {th }}$ instar $R$. prolixus injected with dsRNA. 
Figure 3. Feeding assay following dsRNA injection. ..............................................................72

Figure 4. Feeding assay following injection of antiserum.....................................................74

Figure 5. Results of qPCR analysis, showing the relative expression of the Rhopr-CRF/DHR2B transcript in the CNS of $5^{\text {th }}$ instar $R$. prolixus injected with dsCRFR and dsARG ......77

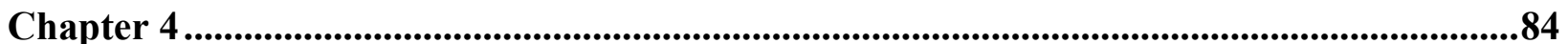

Figure 1. A schematic depicting the known and hypothesized mechanisms underlying the behavioural effects of Rhopr-CRF/DH localized in NSCs in the brain and MTGM. ...........90 


\section{List of Abbreviations}

5-HT:

CNS:

CRF:

DH:

dsRNA:

GPCR:

HPA axis:

MTGM:

NSCs:

PRO:

RNA:

SiRNA:

SOG:

Ucn: 5-hydroxytryptamine (serotonin)

central nervous system

corticotropin-releasing factor

diuretic hormone

double-stranded RNA

G-protein coupled receptor

hypothalamic-pituitary-adrenal axis

mesothoracic ganglionic mass

neurosecretory cells

prothoracic ganglion

RNA interference

small interfering RNA

suboesophageal ganglion

urocortin 


\section{Chapter 1 \\ General Introduction}

\section{Rhodnius prolixus, the disease vector}

Understanding the regulatory pathways and mechanisms controlling vital physiological processes is scientifically important, particularly when it concerns organisms that pose a threat to humans, such as disease vectors. The research advances scientific knowledge but also helps to inform on more effective means of vector control and thereby disease transmission. One such organism is Rhodnius prolixus, a blood-feeding triatomine bug that is a vector of Chagas disease, caused by the parasite Trypanosoma cruzi (World Health Organization (WHO), 2017). The parasite is transmitted following feeding, when contaminated urine and feces enter the human host through the bite or by scratching (Centers for Disease Control and Prevention, 2017). In the early stages following infection, the disease is curable with administration of antiparastic medications; however, the acute symptoms are mild and non-specific, and so can go undetected. In the chronic phase, the disease affects the nervous, cardiovascular, and digestive systems and can be fatal (WHO, 2017). Six to 7 million people are infected worldwide, with most cases occurring in Latin American countries, to which the insect, and therefore the disease, is endemic (WHO, 2017). Migration of infected persons, however, has resulted in a growing number of cases of Chagas disease in North America and Europe, where lack of awareness results in the cardiomyopathy not being recognized, or congenital transmission occurring from mother to child (Bern, 2015).

\section{Corticotropin-releasing factor (CRF), its pathway, and modes of action in the vertebrates}

CRF is a neurohormone involved in the stress response in mammals, modulating vital processes, such as feeding and reproduction, in response to environmental and internal stressors. Hans Selye first described the concept of stress, initially termed "general adaptation syndrome", in the living system in 1936, and theorized that there was a single mediator, later identified as CRF, that triggered the system's adaptive response to stress (Selye, 1936; Stengel and Tache, 2010). Today, it is known that there is an increase in expression of the CRF gene as well as secretion of 
CRF following exposure to stressful stimuli. Two sources of CRF, hypothalamic and central, work concurrently to coordinate an integrated stress response.

CRF's involvement in the neuroendocrine stress response occurs through activation of the hypothalamo-pituitary-adrenal (HPA) axis. CRF is secreted from the hypothalamus, binds to its receptor in the anterior pituitary, from which adrenocorticotropic hormone (ACTH) is released and which in turn drives the release of glucocorticoids from the adrenal cortex. Glucocorticoids adjust metabolism, immune function, growth, reproduction, and behavior, among other biological processes via regulation of gene expression, to maintain homeostasis (Kovacs, 2013; Bale and Vale, 2004). Glucocorticoids also act in a negative feedback loop to inhibit the activity of the HPA axis, reducing CRF transcription (Herman et al., 2012). When glucocorticoid signaling is disrupted, an increase in CRF transcript levels is observed in the paraventricular nucleus (PVN). It has been shown that this negative regulation of CRF by glucocorticoids is present even when the organism in not experiencing stress. Under normal conditions, that is, when the organism is not stressed, CRF neurons are inhibited by GABAergic interneurons, also located in the PVN. This inhibition by GABA has been shown to be removed with injection of GABA antagonists. Thus, sensory information about a stressful stimulus delivered to the PVN either directly or indirectly through the limbic system for example, leads to the inhibition of the GABAergic interneurons and activation of the stress response (Aguilera and Liu, 2012).

Central CRF acts separately to the aforementioned major neuroendocrine response (Aguilera, 1998; Kovacs, 2013) and independently of glucocorticoid signaling, to produce autonomic and behavioural responses to stress. Nevertheless, the two CRF systems (i.e., hypothalamic and central) are related as far as biological stress is concerned. Central CRF is released from neurons projecting from the brain stem, amygdala and septum to the locus coeruleus (LC) and spinal cord, stimulating release of noradrenaline and thereby initiating the fight-or-flight response, increasing heart rate and metabolism, among other physiological expressions, subsequently producing fear and anxiety behaviours. When CRF was overexpressed in rodents, it triggered anxious behaviours, while downregulating gene expression counteracted the effect.

In vertebrates, there are other peptides belonging to the CRF family, called urocortins (Ucn 1, 2, and 3; that share between $26 \%$ and $45 \%$ amino acid sequence identity with CRF), which have been shown to act both analogously and alternatively to CRF in response to stress. Unlike CRF, 
however, they do not appear to be primary signaling molecules that activate the stress response cascades, instead acting as supporting factors. This is based on what is known about their secretion and their receptor. Whether secretion of Ucns endogenously is triggered by the stressful stimulus itself is not clear. Literature to date highlights Ucns 2 and 3 as stress-coping factors, opposing the effects of CRF, reducing blood pressure, and anxiety and arousal behaviours (Suda et al., 2004).

CRF acts through CRF receptor 1 (CRFR1; Ucn1 also exhibits high affinity for this receptor), while Ucns exhibits greater affinity for CRF receptor 2 (CRFR2) (Henckens et al., 2016). Both receptors are class $B, \mathrm{G}$ protein-coupled receptors (GPCRs) and stimulate the cyclic AMP/protein kinase A signaling pathway (Grammatopoulos, 2012). It can be said that the activation of CRFR1 by CRF produces the endocrine, sympathetic, and anxiogenic response to stress. Indeed, it has been shown that when CRFR1 signaling was disrupted or obliterated in rodents and rats, respectively, the presentation of anxious behaviour was prevented. CRFR2, on the other hand, is likely responsible for the previously mentioned homeostatic maintenance through activation by ligands Ucns 2 and 3. Mice in whom CRFR2 was knocked out showed a more pronounced and maladaptive response to stress, including higher levels of glucocorticosteroids, magnified anxiety symptoms, and poorer recovery ability (Henckens et al., 2016). Furthermore, expression of Uen 2 has been shown to increase in the rat pituitary with increased CRF levels (Nemoto et al., 2007), suggesting that activation of CRFR2 is secondary to the stress response initiated through CRFR1, though they must work in conjunction to signal stress in the system and respond accordingly to maintain homeostasis.

\section{A note on the evolution of CRF}

Peptides belonging to the CRF family evolved in metazoans before the time of the deuterostomeprotostome division in evolutionary history, and are conserved across vertebrate and invertebrate species (Lovejoy and de Lannoy, 2013). In invertebrates, one family of diuretic hormones (DHs) shares structural similarity with the mammalian CRF and Ucns, having sequence similarities with both peptide lineages. The functional role of CRF and its paralogues in the maintenance of homeostasis has been described in vertebrates and invertebrates, the former more extensively, highlighting a systemic role in anxiogenic and anxiolytic responses, while the latter is mainly discussed with respect to its osmoregulatory function in insects as a DH. Despite this apparent 
functional variation, the structural conservation of this family of peptides, as well as their common involvement in homeostasis, highlights the significance of their signaling roles in these systems (Lovejoy and de Lannoy, 2013; Lovejoy and Jahan, 2006) and alludes to other potentially parallel actions of CRF in species across the animal kingdom, presumably with respect to the vital perception and management of stress, which, just like the family of peptides, is a correspondingly highly conserved mechanism.

\section{The link between feeding, reproduction, and CRF in vertebrates}

Stress triggers a systemic response; it affects all body systems, and therefore it is conceivable that $\mathrm{CRF}$, the initiator of the stress response, could affect a multitude of biological processes. Feeding and reproduction are two processes that have been extensively described in the context of the stress response and CRF. A multitude of stressors have been shown to reduce food intake, with CRF in particular inhibiting feeding in fish, amphibians, reptiles, birds, and mammals (Carr, 2002). The research to date has described numerous signaling pathways and their components that are involved in CRF's attenuation of feeding.

A molecular mechanism by which CRF modulates food intake in vertebrates is through adipocytokine leptin signaling. Leptin functions as a regulator of energy homeostasis by inducing satiety (Klok et al., 2007). Leptin production and release is stimulated by glucocorticoids, a downstream component in the stress-induced HPA axis activation, and in turn leptin drives negative feedback of CRF (Heiman et al., 1997). When leptin was administered to starved, and therefore stressed mice, it resulted in the normalization of ACTH and glucocorticoid levels (Ahima et al., 1996). A similar effect was observed in mice experiencing restraint stress (Heiman et al., 1997).

A physiological mechanism by which the behavioural manifestation of decreased food intake in response to stress/CRF signaling activation may come about is through changes in gastrointestinal function. Intracerebral injection of CRF, as well as of Ucns 1 and 2, has been shown to inhibit gastric contractions and emptying of a meal through activation of the CRFR2 receptor, with treatment with receptor antagonists reversing this effect (Stengel and Tache, 2010). This suggests that the inhibitory feeding effects are not a result of the initial response following stress perception (i.e., HPA axis activation), but rather part of the secondary response 
initiated by Ucns through CRFR2 to regulate the initial response and maintain homeostasis. This hypothesis is supported by the reported higher potency of Ucns as feeding inhibitors relative to CRF (Gosnell et al., 1983; Hashimoto et al., 2004; Spina et al., 1996).

One of the intermediary factors involved in the context of CRF signaling, gastric motility, and reduced feeding is ghrelin, a hormone involved in digestion. Among ghrelin's functions are appetite stimulation, gastric motility, and metabolism. When Ucn1 was injected into rats it resulted in decreased levels of active ghrelin in circulation, also by activation of CRFR2, since administration of a CRFR1 antagonist did not restore the reduced ghrelin levels stimulated by Ucn1 (Yakabi et al., 2011). This further supports the notion that the anti-feedant effect of stress is the result of CRFR2 activation by Ucns, presumably in response to initial stress response signaling through CRF and CRFR1.

In the context of reproduction, a role for CRF has also been well established in vertebrates. Stress inhibits reproductive processes, and given that CRF is the major player in the stress response, it is implicated as a modulator of these processes. It has been shown that CRF delays puberty onset in female rats, and administration of its receptor antagonist reverses this effect. One of the mechanisms at play in this interaction is the inhibition of gonadotrophin-releasing hormone $(\mathrm{GnRH})$, and thereby inhibition of steroidogenesis and sexual maturation, by CRF (Kinsey-Jones et al., 2010; Kovacs, 2013). The secretion of follicle stimulating hormone (FSH) and luteinizing hormone (LH), both involved in gonadal development, is controlled by $\mathrm{GnRH}$, and therefore activation of the HPA axis and CRF signaling suppresses activity of the hypothalamic-pituitary-gonadal axis. Furthermore, work in rats has shown that Ucn2, which acts through CRFR2, inhibits LH secretion, with administration of the receptor antagonist reversing this effect (Li et al., 2005), suggesting that the effects of stress on reproduction occur via more than one pathway involving activation of both CRFR1 and CRFR2 signaling.

\section{Roles of CRF in invertebrates}

Corticotropin releasing factor-like hormones have been identified and characterized as DHs in a number of insect species in addition to Rhodnius prolixus, including Manduca sexta, Tenebrio molitor, Locusta migratoria and Schistocerca gregaria. Additional roles for CRF have also been investigated in some of these insects. 
In the locust $S$. gregaria, CRF has been found to induce satiety, with insects injected with SchgrCRF prior to feeding taking in a significantly smaller meal relative to controls injected with water (Goldsworthy et al., 2003; van Wielendaele et al., 2012). It is thought that because CRF is released gradually as the insect feeds, that perhaps it signals termination of feeding once the insect is sufficiently full (Gade and Goldsworthy, 2003). A potential mechanism underlying this behaviour is related to CRF's effect on taste perception. Gade and Goldsworthy (2003) found that $\mathrm{CRF}$ induced closure of taste sensing pores on the locust mouthpart. In line with findings in other insects, when the CRF homolog MasDH was injected into Manduca sexta larvae, it resulted in a 70\% decrease in meal size (Keeley et al., 1992). In Drosophila melanogaster, the effect of CRF homolog Dh44 was described with respect to postprandial nutrient sensing, and not satiety. As in R. prolixus (Te Brugge et al., 2001), Dh44 is found in neurosecretory cells (NSCs) in the brain, and these NSCs were found to be sensitive to, and activated by, circulating glucose after feeding. Activation of neurons expressing Dh44 receptors triggered the proboscis extension reflex, which stimulated feeding, as well gut motility and excretion (Dus et al., 2015). Interestingly, there are a separate population of central Dh44 neurons characterized as prescriptive hunger sensors, which, when manipulated, altered the degree of feeding (Dus et al., 2015). This work highlights another role for CRF in feeding, not only as a satiety factor, but also involved in energy balance through glucose homeostasis, a well established role of CRF in vertebrates.

In addition to its role in the modulation of feeding, CRF has been found to have a role in reproductive processes. In L. migratoria, CRF is co-localized with ovary maturating parsin (Locmi-OMP), in NSCs in the brain, and sequence analysis shows these two neurohormones are encoded on the same gene in both L. migratoria and S. gregaria. Further study showed injection of mature S. gregaria females with Schgr-OMP/DH resulted in the production of significantly smaller oocytes, and in reduced levels of ecdysteroids circulating in the haemolymph. Knockdown of Schgr-OMP/DH reversed the adverse effects on feeding and reproduction (van Wielendaele et al., 2012). In D. melanogaster, Dh44 neuron activation was found to delay sperm ejection from the uteri of mature females, while suppression of this neuronal activity expedited the process. Sperm ejection is a mechanism of post-copulatory selection by the female for quality sperm, which suggests that CRF signaling, by mediating retention and storage of sperm by the female, influences the resultant reproductive success of the female and quality of offspring. 


\section{Rhopr-CRF/DH}

A CRF ortholog (Rhopr-CRF/DH) has been identified in $R$. prolixus, where it is, as in some other insects, characterized as a DH. Intake of a huge blood meal, up to ten times its initial body weight, puts great stress on the organism, and the insect responds by initiating diuresis to eliminate the excess water and salt, transmitting Chagas disease in the process (Martini et al., 2007). The rapid diuresis exhibited by $R$. prolixus is, as often found in complex systems and processes, under hormonal control. When hemolymph of a recently fed insect was applied onto isolated Malpighian tubules, potent diuretic activity was observed; that is, stimulation of tubule fluid secretion (Maddrell, 1964; Orchard, 2006, 2009; Te Brugge et al., 2001). Likewise, when CNS tissue homogenates were applied to the isolated tubules, a similar effect was observed, with most of the diuretic activity originating from the posterior lateral neurosecretory cells of the mesothoracic ganglionic mass (MTGM), projecting to the abdominal nerves and releasing the diuretic factors at neurohaemal sites on the nerves (Maddrell, 1964). Initially unidentified, CRF was later determined to be one of the diuretic factors contributing to the 1000 -fold increase in fluid transport by the Malpighian tubules after feeding, working with serotonin to act on the excretory system and orchestrate post-prandial diuresis in R. prolixus (Te Brugge, 2001, Orchard, 2006, 2009).

During feeding, Rhopr-CRF/DH is released from NSCs found in the MTGM; however, the peptide is also found in cell bodies throughout the CNS, including medial NSCs in the brain, suggesting it has additional roles beyond diuresis. There is support for CRF's multifaceted role in $R$. prolixus, particularly in feeding and reproductive processes. The hormone's presence in NSCs and associated neurohaemal organs, from which it is released into the haemolymph means it can have widespread targets. In support of this idea, a Rhopr-CRF/DH receptor is expressed in many tissues in $R$. prolixus (Lee et al., 2016).

\section{Rhopr-CRF/DH signaling}

Rhopr-CRF/DH exerts its effects through action on CRFRs belonging to the secretin family of GPCRs. A variant of one of two CRFRs has been isolated and sequenced in R. prolixus (RhoprCRF/DH-R2B) (Lee et al., 2016). It is a class B1 GPCR, which signals through the cyclic AMP (cAMP)-dependent pathway upon activation by Rhopr-CRF/DH. The downstream targets of this pathway in $R$. prolixus are not known, however, research in mammals has described class B1 
GPCRs, and particularly CRFRs, as regulators of a variety of physiological processes, including feeding, metabolism and growth, diuresis, and reproduction.

Spatial expression analysis has provided insight into the physiological targets of Rhopr-CRF/DH. Expression of Rhopr-CRF/DH-R2B mRNA was observed throughout many tissues of the $5^{\text {th }}$ instar R. prolixus, with the exception of the salivary glands. The highest expression of the receptor was observed in the male immature testes, the foregut and female immature ovaries. Interestingly, there was a significant increase in expression of the receptor in the mature ovaries relative to the immature ovaries of $5^{\text {th }}$ instars (Lee et al., 2016), suggesting that CRF has a reproductive role in the sexually mature female. 


\section{Objectives}

The existing data on CRF drives speculation of its potentially complex, and yet to be explored, role in the physiology of $R$. prolixus. This study aims to provide a comprehensive description of the effects of Rhopr-CRF/DH on the feeding and reproductive parameters through application of physiological and molecular techniques. Therefore, the specific objectives of the study are to describe: 1) the temporal expression of Rhopr-CRF/DH in the $5^{\text {th }}$ instar and adult CNS of $R$. prolixus, using immunohistochemistry and qPCR; 2) the effect of Rhopr-CRF/DH on feeding in 5 th instar and adult $R$. prolixus, using feeding assays; 3 ) the effect of Rhopr-CRF/DH on reproduction in adult $R$. prolixus, using egg-laying assays and in vitro oviduct contraction assays; and 4) the aforementioned parameters following RNAi targeting the Rhopr-CRF/DH transcript. 


\section{References}

Aguilera, G., 1998. Corticotropin Releasing Hormone, Receptor Regulation and the Stress Response. Trends Endocrinol. Metab. 9, 329-336. doi:10.1016/S1043-2760(98)00079-4

Aguilera, G., Liu, Y., 2012. The molecular physiology of CRH neurons. Front. Neuroendocrinol. doi:10.1016/j.yfrne.2011.08.002

Ahima, R.S., Prabakaran, D., Mantzoros, C., Qu, D., Lowell, B., Maratos-Flier, E., Flier, J.S., 1996. Role of leptin in the neuroendocrine response to fasting. Nature. doi:10.1038/382250a0

Bale, T.L., Vale, W.W., 2004. CRF AND CRF R ECEPTORS : Role in Stress Responsivity and Other Behaviors. Annu. Rev. Pharmacol. Toxicol. 44, 525-557.

doi:10.1146/annurev.pharmtox.44.101802.121410

Carr, J. a, 2002. Stress, neuropeptides, and feeding behavior: a comparative perspective. Integr. Comp. Biol. 42, 582-590. doi:10.1093/icb/42.3.582

Dus, M., Lai, J.S.-Y., Gunapala, K.M., Min, S., Tayler, T.D., et al., 2015. Nutrient sensor in the brain directs the action of the brain-gut axis in Drosophila. Neuron 87, 139-51.

doi:10.1016/j.neuron.2015.05.032

Gäde, G., Goldsworthy, G.J., 2003. Insect peptide hormones: A selective review of their physiology and potential application for pest control. Pest Manag. Sci. doi:10.1002/ps.755

Goldsworthy, G.J., Chung, J.S., Simmonds, M.S.J., Tatari, M., Varouni, S., Poulos, C.P., 2003. The synthesis of an analogue of the locust CRF-like diuretic peptide, and the biological activities of this and some C-terminal fragments, in: Peptides. pp. 1607-1613.

doi:10.1016/j.peptides.2003.09.010

Gosnell, B.A., Morley, J.E., Levine, A.S., 1983. A comparison of the effects of corticotropin releasing factor and sauvagine on food intake. Pharmacol. Biochem. Behav. 19, 771-775. doi:10.1016/0091-3057(83)90078-3

Grammatopoulos, D.K., 2012. Insights into mechanisms of corticotropin-releasing hormone receptor signal transduction. Br. J. Pharmacol. doi:10.1111/j.1476-5381.2011.01631.x

Hashimoto, K., Nishiyama, M., Tanaka, Y., Noguchi, T., Asaba, K., Hossein, P.N., Nishioka, T., Makino, S., 2004. Urocortins and corticotropin releasing factor type 2 receptors in the hypothalamus and the cardiovascular system. Peptides. doi:10.1016/j.peptides.2004.05.024

Heiman, M.L., Ahima, R.S., Craft, L.S., Schoner, B., Stephens, T.W., Flier, J.S., 1997. Leptin inhibition of the hypothalamic-pituitary-adrenal axis in response to stress. Endocrinology 138, 3859-3863. doi:10.1210/en.138.9.3859

Henckens, M.J.A.G., Deussing, J.M., Chen, A., 2016. Region-specific roles of the corticotropinreleasing factor-urocortin system in stress. Nat. Rev. Neurosci. 17, 636-651.

doi:10.1038/nrn.2016.94 
Herman, J.P., Mcklveen, J.M., Solomon, M.B., Carvalho-Netto, E., Myers, B., 2012. Neural regulation of the stress response: Glucocorticoid feedback mechanisms. Brazilian J. Med. Biol. Res. doi:10.1590/S0100-879X2012007500041

Keeley, L.L., Chung, J.S., Hayes, T.K., 1992. Diuretic and antifeedant actions by Manduca sexta diuretic hormone in lepidopteran larvae. Experientia 48, 1145-1148. doi:10.1007/BF01948010

Kinsey-Jones, J.S., Li, X.F., Knox, A.M.I., Lin, Y.S., Milligan, S.R., Lightman, S.L., O’Byrne, K.T., 2010. Corticotrophin-releasing factor alters the timing of puberty in the female rat. J. Neuroendocrinol. 22, 102-109. doi:10.1111/j.1365-2826.2009.01940.x

Klok, M.D., Jakobsdottir, S., Drent, M.L., 2007. The role of leptin and ghrelin in the regulation of food intake and body weight in humans: a review. Obes. Rev. 8, 21-34. doi:10.1111/j.1467789X.2006.00270.x

Kovács, K.J., 2013. CRH: The link between hormonal-, metabolic- and behavioral responses to stress. J. Chem. Neuroanat. 54, 25-33. doi:10.1016/j.jchemneu.2013.05.003

Lee, H.R., Zandawala, M., Lange, A.B., Orchard, I., 2016. Isolation and characterization of the corticotropin-releasing factor-related diuretic hormone receptor in Rhodnius prolixus. Cell. Signal. 28, 1152-1162. doi:10.1016/j.cellsig.2016.05.020

Lee, K.M., Daubnerov??, I., Isaac, R.E., Zhang, C., Choi, S., Chung, J., Kim, Y.J., 2015. A neuronal pathway that controls sperm ejection and storage in female drosophila. Curr. Biol. 25, 790-797. doi:10.1016/j.cub.2015.01.050

Li, F.X., Bowe, J.E., Lightman, S.L., O’Byrne, K.T., 2005. Role of corticotropin-releasing factor receptor-2 in stress-induced suppression of pulsatile luteinizing hormone secretion in the rat. Endocrinology 146, 318-322. doi:10.1210/en.2004-0950

Longo, D.L., Bern, C., 2015. Chagas’ Disease. N. Engl. J. Med. 373, 456-466. doi:10.1056/NEJMra1410150

Lovejoy, D.A., de Lannoy, L., 2013. Evolution and phylogeny of the corticotropin-releasing factor (CRF) family of peptides: Expansion and specialization in the vertebrates. J. Chem. Neuroanat. doi:10.1016/j.jchemneu.2013.09.006

Lovejoy, D.A., Jahan, S., 2006. Phylogeny of the corticotropin-releasing factor family of peptides in the metazoa. Gen. Comp. Endocrinol. doi:10.1016/j.ygcen.2005.11.019

Maddrell, S.H., 1964. Excretion in the Blood-Sucking Bug, Rhodnius Prolixus Stal. 3. the Control of the Release of the Diuretic Hormone. J. Exp. Biol. 41, 459-472.

Martini, S. V., Nascimento, S.B., Morales, M.M., 2007. Rhodnius prolixus Malpighian tubules and control of diuresis by neurohormones. An. Acad. Bras. Cienc. 79, 87-95. doi:10.1590/S0001-37652007000100011 
Nemoto, T., Iwasaki-Sekino, A., Yamauchi, N., Shibasaki, T., 2010. Role of urocortin 2 secreted by the pituitary in the stress-induced suppression of luteinizing hormone secretion in rats. Am. J. Physiol. Endocrinol. Metab. 299, E567-75. doi:10.1152/ajpendo.00163.2010

Orchard, I., 2006. Serotonin: A coordinator of feeding-related physiological events in the bloodgorging bug, Rhodnius prolixus. Comp. Biochem. Physiol. - A Mol. Integr. Physiol. doi:10.1016/j.cbpa.2005.11.010

Orchard, I., 2009. Peptides and serotonin control feeding-related events in Rhodnius prolixus. Front. Biosci. - Elit. 1 E, 250-262. doi:24 [pii]

Selye, H., 1936. A Syndrome Produced by Diverse Nocuous Agents. J. Neuropsychiatry Clin. Neurosci. 10, 230-231. doi:10.1038/138032a0

Spina, M., Merlo-Pich, E., Chan, R.K., Basso, A.M., Rivier, J., Vale, W., Koob, G.F., 2015. Appetite-suppressing effects of urocortin, a CRF-related neuropeptide. Science (80-. ). 273, 1561-1564. doi:10.1126/science.273.5281.1561

Stengel, A., Taché, Y., 2014. CRF and urocortin peptides as modulators of energy balance and feeding behavior during stress. Front. Neurosci. doi:10.3389/fnins.2014.00052

Suda, T., Kageyama, K., Sakihara, S., Nigawara, T., 2004. Physiological roles of urocortins, human homologues of fish urotensin I, and their receptors. Peptides.

doi:10.1016/j.peptides.2004.03.027

Te Brugge, V.A., Nässel, D.R., Coast, G.M., Schooley, D.A., Orchard, I., 2001. The distribution of a kinin-like peptide and its co-localization with a CRF-like peptide in the blood-feeding bug, Rhodnius prolixus. Peptides 22, 161-173. doi:10.1016/S0196-9781(00)00373-9

van Wielendaele, P., Dillen, S., Marchal, E., Badisco, L., Vanden Broeck, J., 2012. CRF-like diuretic hormone negatively affects both feeding and reproduction in the desert locust, Schistocerca gregaria. PLoS One 7. doi:10.1371/journal.pone.0031425

Yakabi, K., Noguchi, M., Ohno, S., Ro, S., Onouchi, T., Ochiai, M., Takabayashi, H., Takayama, K., Harada, Y., Sadakane, C., Hattori, T., 2011. Urocortin 1 reduces food intake and ghrelin secretion via CRF(2) receptors. Am. J. Physiol. Endocrinol. Metab. 301, E72-82. doi:10.1152/ajpendo.00695.2010 


\section{Chapter 2 \\ The involvement of Rhopr-CRF/DH in feeding and reproduction in the blood-gorging insect Rhodnius prolixus}

Mollayeva S, Orchard I, Lange AB. The involvement of Rhopr-CRF/DH in feeding and reproduction in the blood-gorging insect Rhodnius prolixus. Gen. Comp. Endocrinol. (2017), http://dx.doi.org/10.1016/j.ygcen.2017.07.005

\section{Abstract}

Rhodnius prolixus is a blood-gorging insect and a vector for human Chagas disease. The insect transmits the disease following feeding, when it excretes urine and feces contaminated with the Trypanosoma cruzi parasite. A corticotropin-releasing factor-like peptide acts as a diuretic hormone in R. prolixus (Rhopr-CRF/DH); however, its distribution throughout the insect's central nervous system (CNS) and the expression of its receptor in feeding-related tissue as well as the female reproductive system suggests a multifaceted role for the hormone beyond that of diuresis. Here we investigate the involvement of Rhopr-CRF/DH in feeding and reproduction in R. prolixus. Immunohistochemistry of the CNS showed diminished CRF-like staining in neurosecretory cells (NSCs) of the mesothoracic ganglionic mass (MTGM) immediately following feeding, and partial restocking of those same cells two hours later, indicating Rhopr$\mathrm{CRF} / \mathrm{DH}$ stores in these regions are involved in feeding. The results of the temporal qPCR analysis were consistent with the immunohistochemical findings, showing an increase in RhoprCRF/DH transcript expression in the MTGM immediately after feeding, presumably capturing the restocking of Rhopr-CRF/DH in the lateral NSCs following release of the peptide during feeding. Elevating haemolymph Rhopr-CRF/DH titres by injection of Rhopr-CRF/DH prior to feeding resulted in the intake of a significantly smaller blood meal in $5^{\text {th }}$ instars and adults without an apparent effect on the rate of short-term diruesis. When adult females were injected with Rhopr-CRF/DH, they also produced and laid significantly fewer eggs. Finally, in vitro oviduct contraction assays illustrate that Rhopr-CRF/DH inhibits the amplitude of contractions of the lateral oviducts, highlighting a potential mechanism via which the hormone diminishes reproductive capacity. To conclude, the study of the Rhopr-CRF/DH pathway, its components and mechanisms of action, has implications for vector control by highlighting targets to alter feeding, diuresis, and reproduction of this disease vector. 
Key words: stress, diuresis, satiety, egg production, triatomine

\section{Introduction}

Rhodnius prolixus is a blood-gorging insect and a vector for human Chagas disease, caused by transmission of the parasite Trypanosoma cruzi. The disease affects the nervous, cardiovascular, and digestive systems and can be fatal in severe cases (World Health Organization, 2017). It is estimated that 6 to 7 million people are currently infected worldwide, with most cases occurring in Central and South America where R. prolixus is endemic (World Health Organization, 2017). Disease transmission occurs after feeding, when the insect takes a huge blood meal, up to ten times its initial body weight, triggering rapid diuresis to eliminate the excess water and salts (Martini et al., 2007). The T. cruzi-contaminated urine or feces enters the human host through the feeding wound or following scratching (Centers for Disease Control and Prevention, 2017). The haemolymph of a recently fed insect, as well as tissue homogenates from the mesothoracic ganglionic mass (MTGM) of the central nervous system (CNS), have been shown to potently stimulate Malpighian tubule secretion, leading to diuresis (Te Brugge et al., 2001; Coast et al., 2002; Orchard, 2006, 2009). Initially unidentified, a corticotropin-releasing factor-like hormone (Rhopr-CRF/DH) was later determined to be one of the diuretic hormones in R. prolixus contributing to the 1000-fold increase in fluid transport by the Malpighian tubules after feeding, working in concert with serotonin to act on the excretory system and orchestrate post-prandial diuresis (Te Brugge, 2001; Coast et al., 2002; Orchard, 2006, 2009; Martini et al., 2007).

The mammalian corticotropin-releasing factor (CRF) is a neurohormone involved in the stress response, modulating a number of physiological processes in response to stressors, including feeding and reproduction (Majzoub, 2006). There is reason to believe that its ortholog in $R$. prolixus likewise has multifaceted roles, beyond diuresis (Lee et al., 2016).

Corticotropin releasing factor-like hormones have been identified and characterized as diuretics in a number of other insect species, including Manduca sexta, Tenebrio molitor, Locusta migratoria and Schistocerca gregaria (Coast et al., 2002; van Wielendaele et al., 2012). Additional roles for the insect CRF have been investigated in some of these insects. In $S$. gregaria, $\mathrm{CRF} / \mathrm{DH}$ has been found to induce satiety, with insects injected with the peptide prior 
to feeding taking in a significantly smaller meal relative to water-injected controls (van Wielendaele et al., 2012). It is conjectured that because CRF/DH is released into the haemolymph as the insect feeds, that it may signal the end of feeding. In addition to its role in the regulation of feeding, CRF has been found to have a role in reproductive processes. In $L$. migratoria, Locmi-CRF/DH is co-localized with ovary maturating parsin (OMP) in neurosecretory cells (NSCs) in the pars intercerebralis, and sequence analysis shows these two neurohormones are encoded on the same gene in both L. migratoria and S. gregaria (Girardie et al., 1998; van Wielendaele et al., 2012). Further study showed that injection of mature $S$. gregaria females with CRF/DH resulted in the production of significantly smaller oocytes, and in reduced levels of ecdysteroids circulating in the haemolymph (van Wielendaele et al., 2012). Knockdown of Schgr-OMP reversed the adverse effects on feeding and reproduction.

During feeding, Rhopr-CRF/DH is released from lateral NSCs of the MTGM to initiate diuresis in R. prolixus (Maddrell, 1964; Te Brugge et al., 2001; Orchard, 2009; Lee et al., 2016); however, this peptide is also found in medial NSCs in the brain, with axons projecting to the neurohaemal organ, the corpus cardiacum (CC) (Te Brugge et al., 1999). The NSCs of the MTGM release Rhopr-CRF/DH from neurohaemal sites on abdominal nerves into the haemolymph and can therefore have widespread targets, in addition to those related to diuresis. A Rhopr-CRF/DH receptor is expressed in many tissues in R. prolixus, with pronounced expression observed in the testes, the foregut, and the ovaries of $5^{\text {th }}$ instars (Lee et al., 2016). In adults, a significant increase in expression is observed in the mature ovaries, relative to the immature ovaries of 5th instars, suggesting Rhopr-CRF/DH's activity increases in the sexually mature female. The existing data on CRF drives speculation of its potentially complex, and yet to be explored, role in the physiology of $R$. prolixus.

Thus, this study aimed to describe (1) the time course of expression of Rhopr-CRF/DH from preto post-feeding through immunohistochemistry and qPCR analysis; (2) the effect of Rhopr$\mathrm{CRF} / \mathrm{DH}$ on feeding behaviour through feeding assays; and (3) the effect of Rhopr-CRF/DH on reproductive processes through egg-laying assays and in vitro oviduct contraction assays. Given the current literature we hypothesized that Rhopr-CRF/DH would exhibit inhibitory effects on both feeding and reproduction in $R$. prolixus. Given that serotonin works with Rhopr-CRF/DH to modulate feed-related physiological processes, it was tested in additional to Rhopr-CRF/DH in the feeding assays. Serotonin-like immunoreactivity has been observed in tissues associated with 
feeding (e.g., salivary glands, crop, gut, and cuticle) and staining has been shown to decrease at neurohaemal sites on the abdominal nerves following feeding, suggesting release of serotonin into the haemolymph during a blood meal, as is the case with Rhopr-CR/DH. Furthermore, serotonin is also classified as a diuretic hormone, acting additively with Rhopr-CRF/DH to stimulate Malpighian tubule secretion during post-prandial diuresis. Finally, serotonin has been shown to induce cuticle plasticization in response to a blood meal (Orchard, 2006).

\section{Materials and Methods}

\subsection{Animals}

Newly-emerged juvenile ( $5^{\text {th }}$ instar) and adult $R$. prolixus, approximately 1.5 months post-feed on defibrinated rabbit blood (Cedarlane, Burlington, CA) as $4^{\text {th }}$ and $5^{\text {th }}$ instars, respectively, were taken from a colony maintained at $25^{\circ} \mathrm{C}$ and high humidity $(50 \%)$.

\subsection{Immunohistochemistry}

Fixation and staining were performed as described in Te Brugge et al. (1999). Insects $\left(5^{\text {th }}\right.$ instars and adults) were dissected in physiological saline $\left(150 \mathrm{mM} \mathrm{NaCl}, 8.6 \mathrm{mM} \mathrm{KCl}, 2 \mathrm{mM} \mathrm{CaCl}_{2}, 4\right.$ $\mathrm{mM} \mathrm{NaHCO} 3,34 \mathrm{mM}$ glucose, $8.5 \mathrm{mM} \mathrm{MgCl}_{2}, 5 \mathrm{mM}$ HEPES, $\mathrm{H}_{2} \mathrm{O}, \mathrm{pH}$ 7), their CNSs removed and fixed in $2 \%$ paraformaldehyde for 1 hour at room temperature. After fixation, the tissues were washed in phosphate-buffered saline (PBS) before being incubated in 4\% Triton for 1 hour at room temperature. The tissues were again washed in PBS and then transferred to primary antiserum, containing a polyclonal antisera raised in rabbit against the L. migratoria CRF-like hormone (Locmi-DH) at a concentration of 1:1000 in 0.4\% Triton and normal goat serum (NGS). The tissues were kept in the primary antiserum solution on a rotator for 48 hours at $4{ }^{\circ} \mathrm{C}$. After another round of washing, the tissues were placed in secondary antibody, a 1:600 Cy3labelled sheep anti-rabbit immunoglobulin in NGS and PBS. Control tissues were prepared following the same protocol with the primary antiserum solution pre-absorbed with $10^{-3} \mathrm{M}$ Rhopr-CRF/DH for 48 hours.

Confocal images were analyzed using ImageJ Software (Ferreira and Rasband, 2012) to quantify changes in Rhopr-CRF/DH-like expression (i.e., staining) from pre- to post-feed ( $0 \mathrm{~h}$ and $2 \mathrm{~h}$ ). Staining intensity was determined by tracing the stained region of the medial NSCs in the brain and the lateral NSCs in the MTGM and assigning grayscale values ranging from 0 (minimum 
intensity) to 255 (maximum intensity). To ensure accuracy, all images were treated consistently with respect to scale and number of slices in the Z-stacks.

\subsection{Feeding assay}

Fifth instars were injected with $1 \mu \mathrm{L} 10^{-4} \mathrm{M}$ Rhopr-CRF/DH or physiological saline and weighed to record pre-feed weight. Adults were injected with $2 \mu \mathrm{L} 10^{-4} \mathrm{M}$ Rhopr-CRF/DH or physiological saline and also weighed prior to feeding. Twenty-four or 2-3 hours after injection, insects were fed on defibrinated rabbit blood for 20 minutes. This is the typical time for bloodgorging and whilst diuresis is underway during this time, the insect does not eliminate any urine until feeding has ceased. Immediately after feeding, insects were weighed to measure the size of the meal. They were weighed again 30 minutes later, and then at hourly intervals for four hours to record rate of diuresis.

\subsection{Egg-laying assay}

Fifth instars were sexed, keeping males and females in separate jars. They were then fed as $5^{\text {th }}$ instars and left in an incubator at $25^{\circ} \mathrm{C}$ following a 12-hour light/dark cycle. One to 2 weeks after emergence into adults, they were again fed and returned to the incubator. Seventy-two hours later, males and females were placed into vials for mating ( 1 female: 2 males). Another 48 hours later, males were removed from the vials. Females were left alone in the vials and the following day were injected with $2 \mu \mathrm{L} 10^{-3} \mathrm{M}$ Rhopr-CRF/DH or physiological saline. The number of eggs laid by control and experimental females was recorded over a period of 8 days. On the last day, females were dissected to count the number of eggs present in their ovaries, oviducts, and bursa.

\subsection{Oviduct contraction assay}

Contraction assays were carried out as described in Sedra et al. (2015). Mature females were dissected in physiological saline, exposing their reproductive tissue. The tissue was tied around the common oviduct with silk thread just below the spermatheca, after which cuts were made posterior to the knot as well as anterior at the junction where the ovary meets the oviduct to isolate the lateral and common oviducts for the measurement of muscle contraction. The isolated tissue was placed in a Sylgard-coated dish, immersed in $200 \mu \mathrm{L}$ physiological saline and pinned at the free ends of the oviducts. The other end of the thread was tied to a force transducer, which was connected to an amplifier. The amplified signal was recorded through PicoScope 2200 
(PicoTechnology, St. Neots, UK). After the preparation was stabilized in a bath containing 200 $\mu \mathrm{L}$ of saline, half of the saline $(100 \mu \mathrm{L})$ was replaced with a range of concentrations $\left(2 \times 10^{-13} \mathrm{M}\right.$ to $2 \times 10^{-5} \mathrm{M}$ ) of Rhopr-CRF/DH for 200 seconds. Voltage measurements were converted to force (mg) using weights to compare force of contractions in saline and in Rhopr-CRF/DH.

\subsection{RTqPCR}

The CNS was dissected from $5^{\text {th }}$ instar unfed and fed $(0 \mathrm{~h}, 2 \mathrm{~h}, 4 \mathrm{~h}, 8 \mathrm{~h}$, and $24 \mathrm{~h}$ post-fed) insects. Brain, suboesophageal ganglion (SOG) and prothoracic ganglion (PRO) were analyzed separately from the MTGM. The tissues were lysed and RNA extracted using the Total RNA Minipreps Superkit (Bio Basic Canada Inc., Markham, ON, Canada). Complementary DNA (cDNA) was synthesized using the Applied Biosystems High Capacity cDNA Reverse Transcription Kit (Thermo Fisher Scientific Baltics UAB, Vilnius, Lithuania). The cDNA was used at a concentration of 5ng, together with SsoAdvanced Universal SYBR Green Supermix (Bio-Rad Laboratories Ltd., Mississauga, ON, Canada), to amplify Rhopr-CRF/DH transcript and reference gene transcripts (beta-actin and ribosomal protein 49). The primers used are listed in Table 1 (Sigma-Aldrich Canada Co., Oakville, ON, Canada). All primer efficiencies were $>90 \%$. The reactions were completed on the Mx4000 Quantitative PCR System (Stratagene, Mississauga, ON, Canada). Temporal expression of Rhopr-CRF/DH transcript was determined using the delta-delta $\mathrm{Ct}$ method, calculating expression at each time point post-fed relative to pre-fed levels. Three biological replicates were performed, with three technical replicates for each condition in every trial including a negative control without template cDNA.

Table 1. Primers for qPCR.

\begin{tabular}{ll}
\hline Target & Sequence $\left(5^{\prime}-3^{\prime}\right)$ \\
\hline Rhopr-CRF/DH & Fw: CAACCACCATTACAGGAATC \\
& Rv: GTCAGCCTGTTTGTATGTCG \\
\hline Rhopr-beta-actin & Fw: TCATCTCACCAATCTAACCCAC \\
& Rv: CATTCCTACCATTACACCCTGAT \\
\hline Rhopr-rp49 & Fw: AGGAGAAATTGGCGCAAG \\
& Rv: GAAACCAGTAGGAAGCATGTGT \\
\hline
\end{tabular}




\subsection{Statistical analyses}

All graphs were created with Graph Pad Prism 7 (www.graphpad.com). Immunohistochemistry quantitative data were analyzed with a one-way ANOVA followed by the Tukey's post-hoc test to determine intensity differences over time after feeding. Rhopr-CRF/DH transcript expression using qPCR was analyzed with a one-way ANOVA followed with Bonferroni's test to compare expression at each time point to unfed expression in the brain and the MTGM. Feeding assay data were analyzed with the Student's t-test to compare post-fed body weights between treatment and control groups. Rate of weight loss after feeding, as an indicator of rate of diuresis, was analyzed by fitting the data with regression lines and comparing the slopes for significant differences using an F-test,. Egg-laying assay data were analyzed with a two-way ANOVA followed with Bonferroni's test to determine differences between treatment and control groups in egg-laying over 8 days and differences in number of eggs found in the ovaries, oviducts, and bursa. Oviduct contraction assay data were also analyzed with a two-way ANOVA and Bonferroni's test to determine differences in contraction amplitude relative to saline with application of a range of Rhopr-CRF/DH doses.

\section{Results}

\subsection{CRF/DH-like immunostaining in the CNS}

Rhopr-CRF/DH-like staining was most pronounced in the medial NSCs of the brain (15 bilaterally paired cell bodies), the posterior lateral NSCs of the MTGM (7 bilaterally paired cell bodies), and in neuronal processes along the $\mathrm{CC}$ and the abdominal nerves (Fig. 1, Fig. 2) in unfed $5^{\text {th }}$ instar $R$. prolixus. In unfed adult insects, the distribution of staining in the brain and MTGM resembled that of $5^{\text {th }}$ instars ( 9 bilaterally paired cell bodies in the brain and 7 bilaterally paired cells bodies in the MTGM). Staining in the CC and the abdominal nerves appeared consistent across insect stages. In the prothoracic ganglion (PRO), however, differences in staining were more apparent ( 7 medial and lateral paired cell bodies stained in $5^{\text {th }}$ instars and 4 lateral pairs cell bodies stained in the adults) (Fig. 1, Fig. 2). 
Figure 1. CRF-like immunostaining of $5^{\text {th }}$ instar (left) and adult (right) $R$. prolixus CNS. Staining is apparent in cells and processes throughout the CNS including the medial NSCs (circled) of the brain and lateral NSCs (circled) of the MTGM, as well as in neurohaemal sites on the CC (arrows) and on the abdominal nerves (arrows), in both insect stages. Preparations shown are most representative of an $\mathrm{n}$ of 10 . Scale bars $=100 \mu \mathrm{M}$. 
$5^{\text {th }}$ instar

Brain

CC

PRO

MTGM

Abdominal nerves
Adult

21
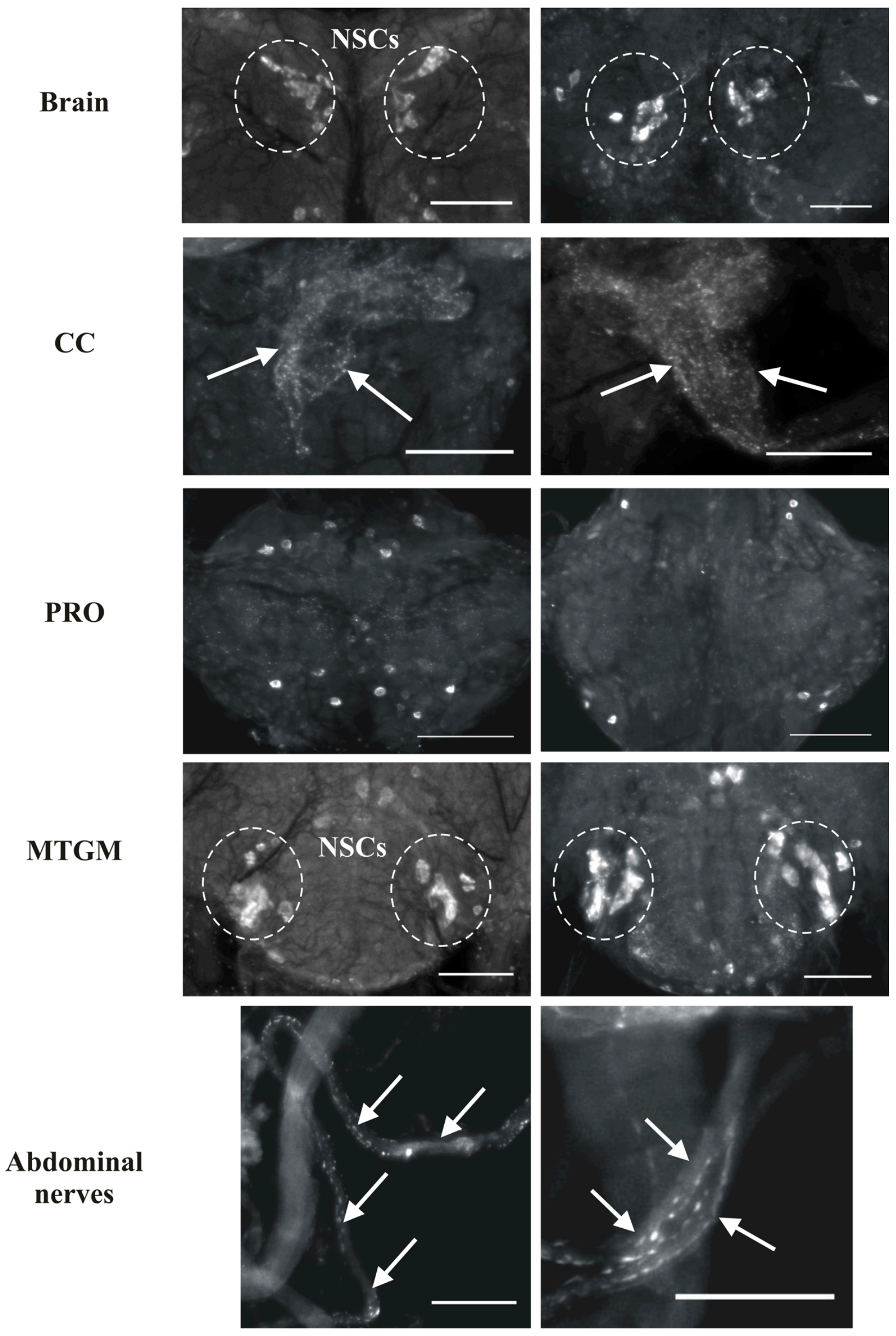
Figure 2. Composite maps of cells that stained consistently for CRF-like immunoreactivity in the CNS of $5^{\text {th }}$ instars (left) and adults (right). 

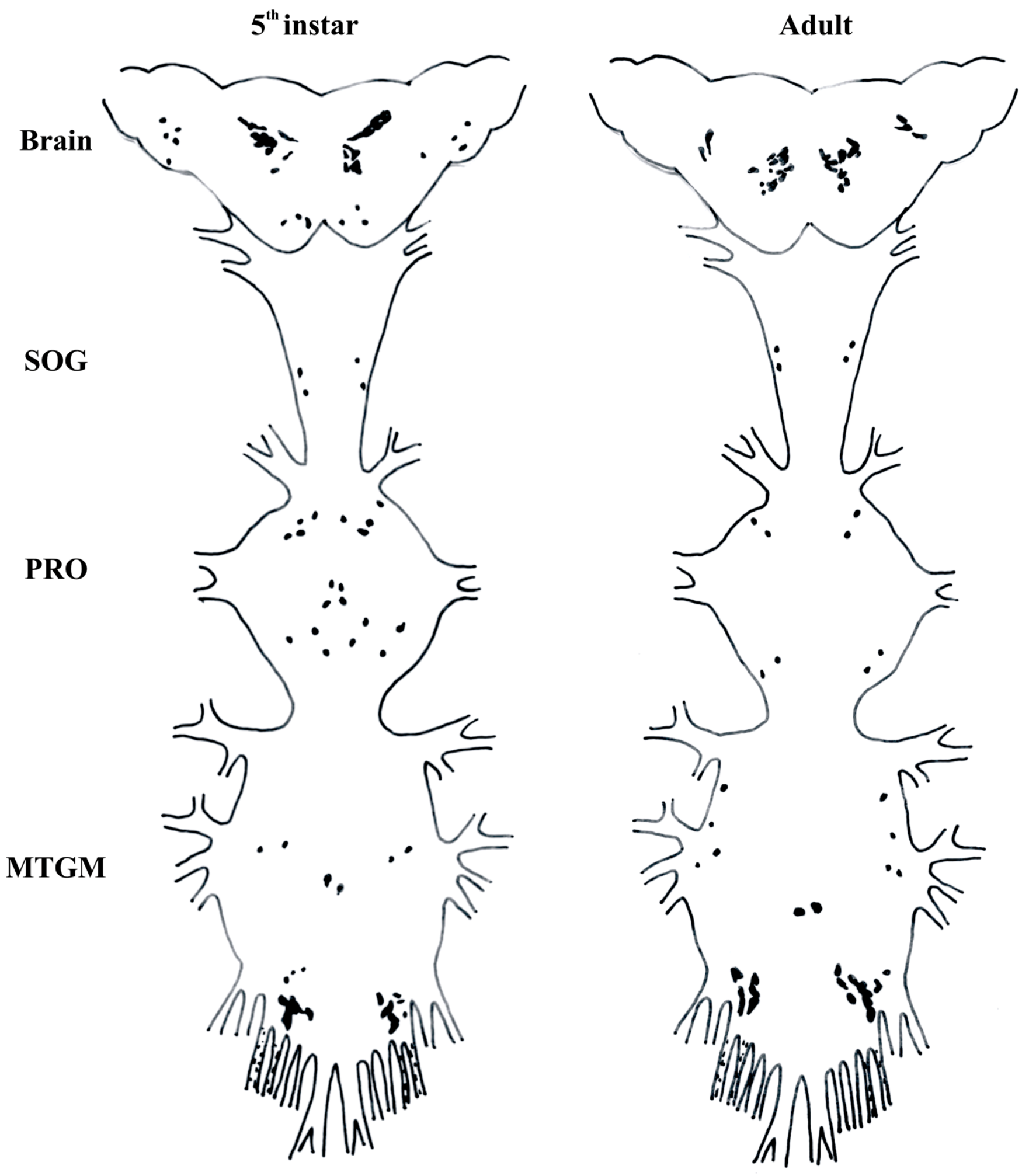


\subsection{CRF-like immunostaining in the CNS after feeding}

Time-course immunohistochemistry of $5^{\text {th }}$ instar CNS $(n=4-6 /$ time point $)$ revealed changes in CRF-like staining in the medial NSCs of the brain and the lateral NSCs of the MTGM from preto post-feed. Fluorescence intensity of cell bodies, quantified with grayscale values, was significantly diminished in both the medial and lateral NSCs immediately after feeding (Fig. 3). Two hours later, the cell bodies were in the process of restocking, with intensity of staining in the brain and MTGM increasing significantly from post-feed levels (Fig. 3). Staining of neuronal

processes in the $\mathrm{CC}$ and on the abdominal nerves appeared to diminish immediately after feeding when compared to pre-feed levels. Two hours later, staining resembled pre-feed levels (Fig. 4).

\section{3 qPCR: expression of Rhopr-CRF/DH transcript in the CNS after feeding}

Expression of the Rhopr-CRF/DH transcript increased in the MTGM immediately after feeding (i.e., 0 hours) relative to expression in the MTGM of unfed insects. The expression then returned to unfed expression levels at 2 and 4 hours after feeding, and increased again at 8 and 24 hours after cessation of feeding. Changes in expression of the Rhopr-CRF/DH transcript in the brain, SOG, and PRO were not apparent from pre- to post-feed and throughout the time course examined (Fig. 5). 
Figure 3. Time-course of CRF-like immunostaining in $5^{\text {th }}$ instar $R$. prolixus.

(A) Mean fluorescence intensity (grayscale values, arbitrary units) in medial NSCs in the brain (left) and lateral NSCs in the MTGM (right) relative to time of feeding. There is a significant decrease in total fluorescence in the medial NSCs of brain and posterior lateral NSCs of the MTGM from pre- to post-feed (one-way ANOVA, ${ }^{* *} \mathrm{p} \leq 0.01$ ). Two hours after feeding, the NSCs in the brain have almost completely been restocked (one-way ANOVA, ${ }^{*} p \leq 0.05$ ). Error bars represent standard error of the mean. (B) Confocal images of the medial NSCs in the brain (left column) and the posterior lateral NSCs in the MTGM (right column) at three time points relative to the feed. Scale bars $=100 \mu \mathrm{M}$. 

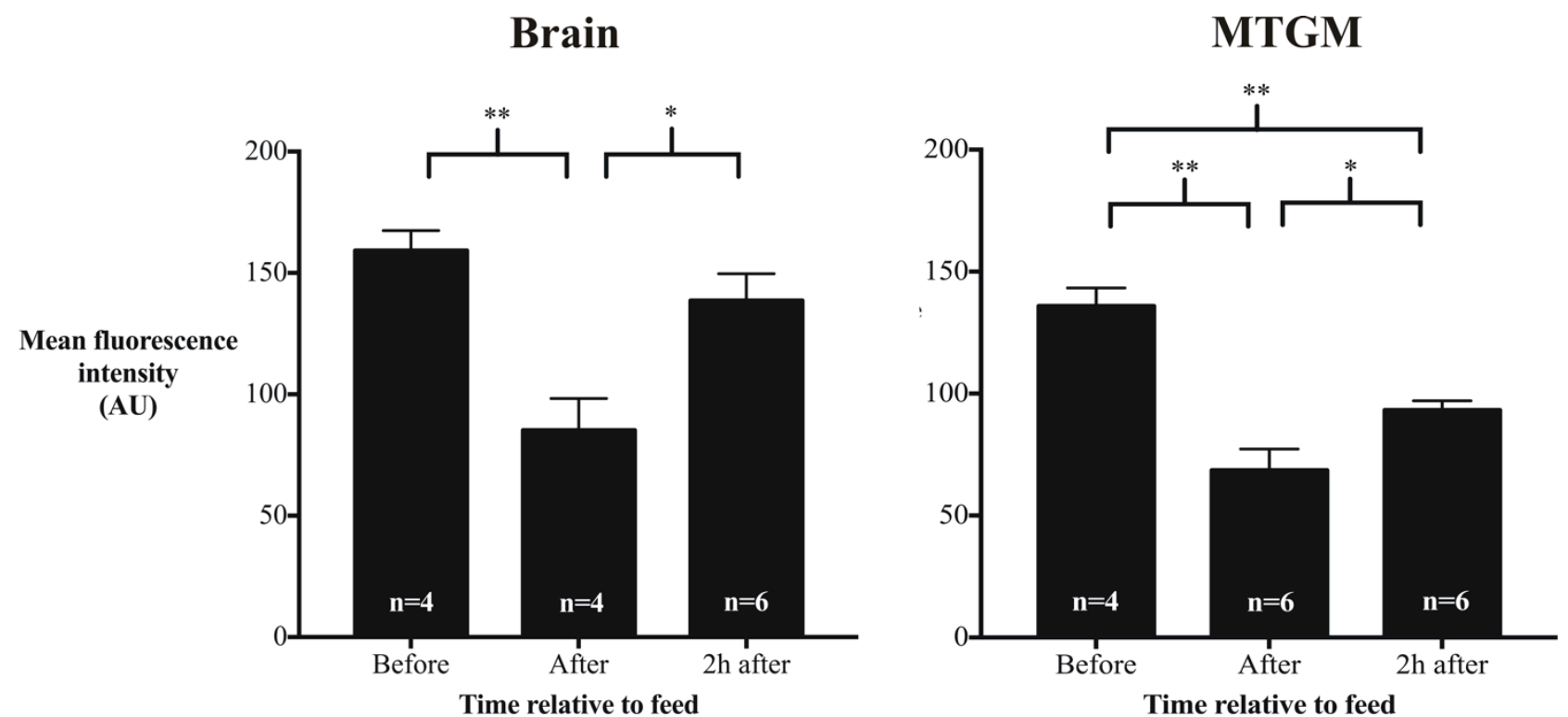

\section{Before}

After
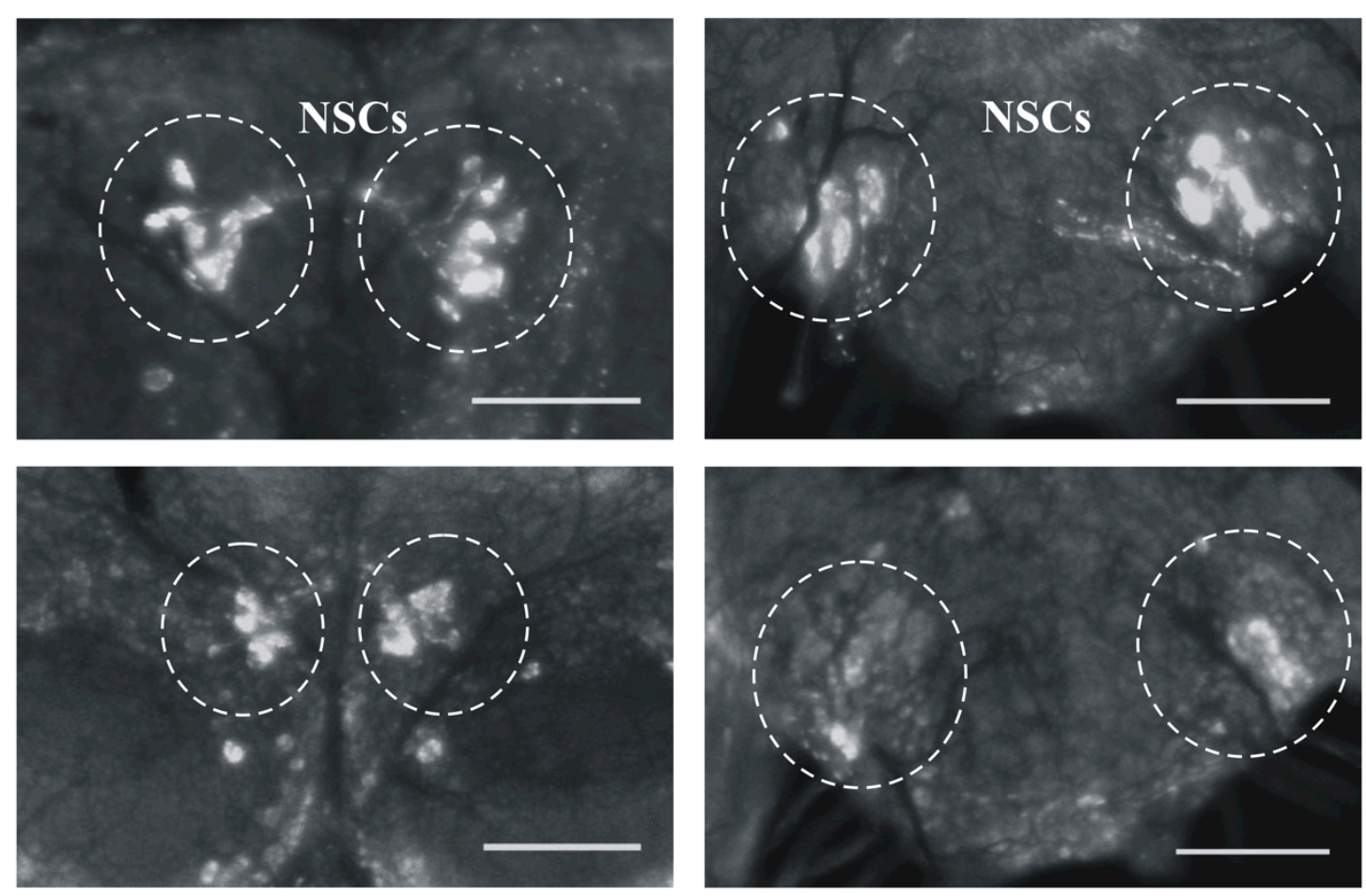

\section{2h after}
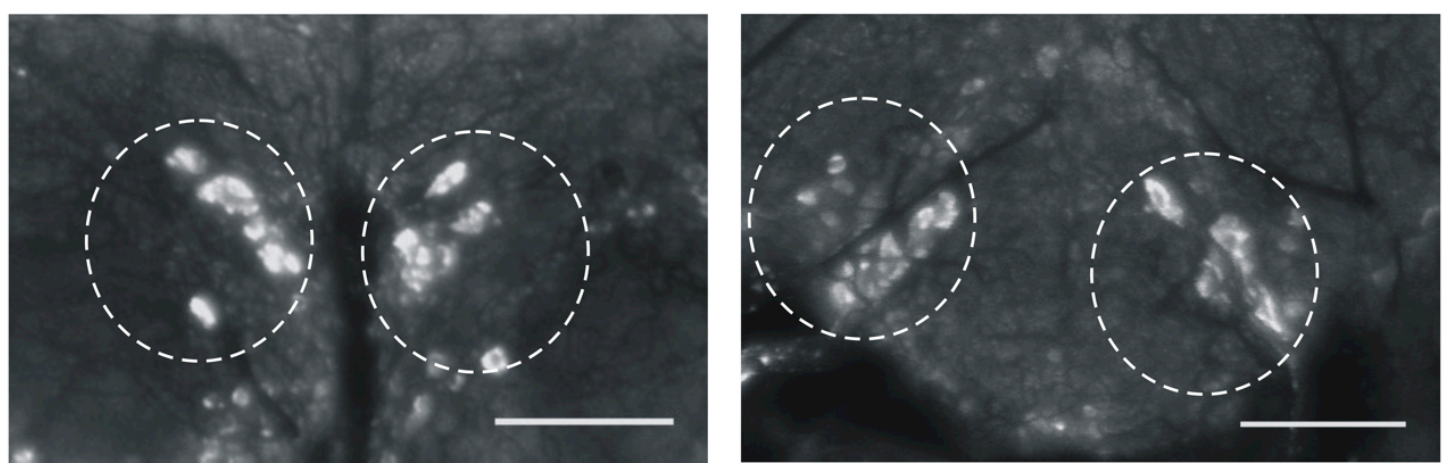
Figure 4. Time-course of CRF-like immunostaining in $5^{\text {th }}$ instar $R$. prolixus before feeding, just after feeding and $2 \mathrm{~h}$ after feeding.

Staining of neuronal processes in the $\mathrm{CC}$ and on the abdominal nerves is diminished immediately after feeding, and intensifies by 2 hours after feeding, resembling pre-feeding staining. Scale bars $=100 \mu \mathrm{M}$. 
Before
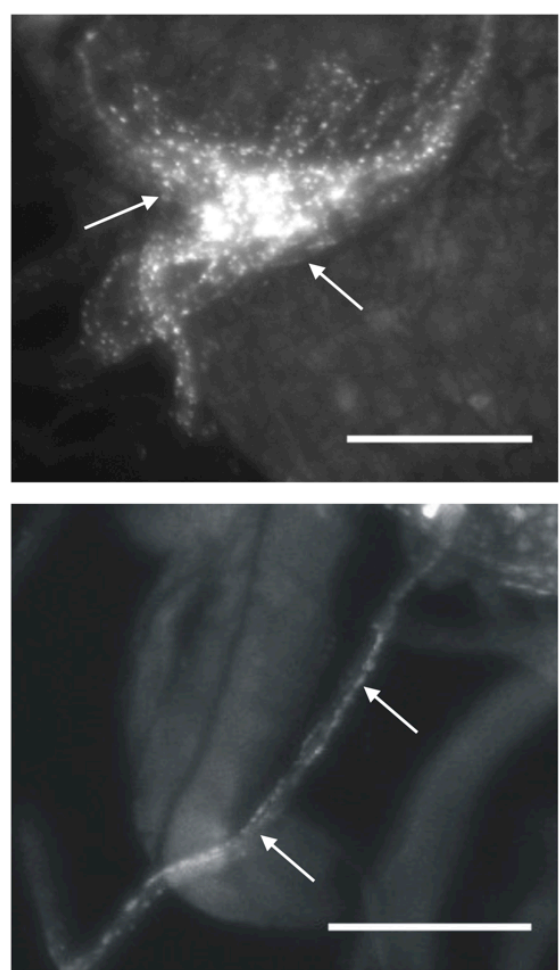

After
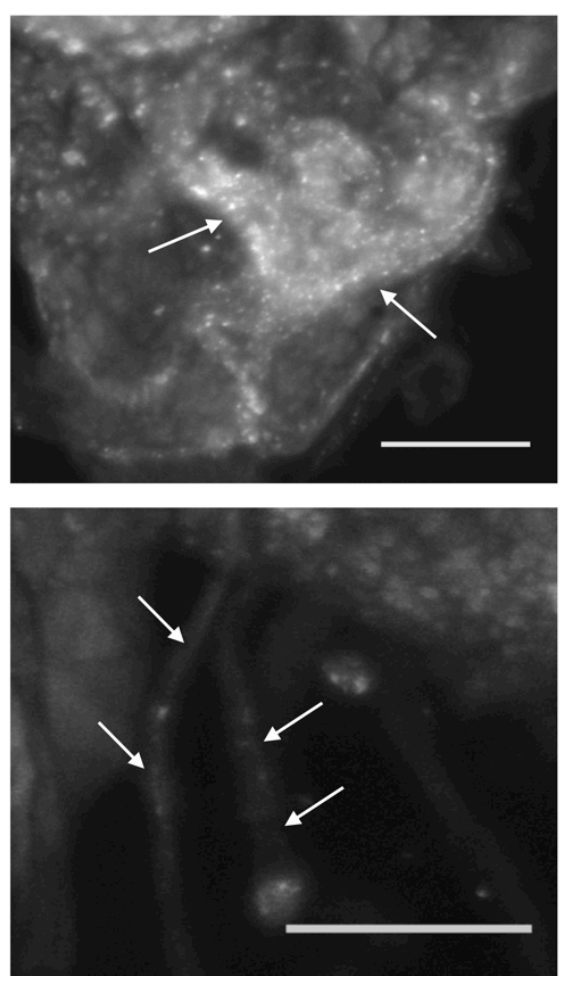

2h after
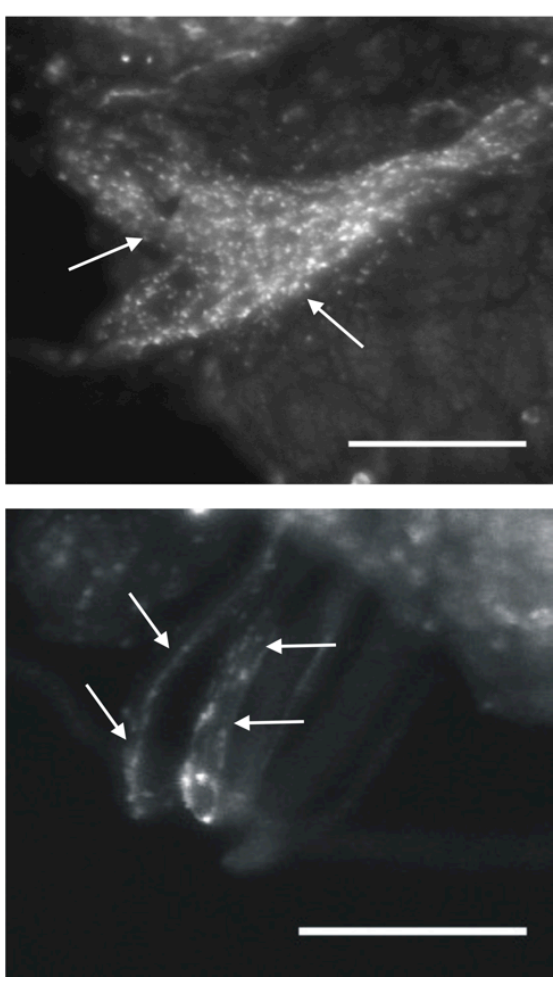

Abdominal nerves 
Figure 5. Temporal expression of the Rhopr-CRF/DH transcript in the brain and MTGM of $5^{\text {th }}$ instar $R$. prolixus.

Expression appears to increase in the MTGM immediately after feeding relative to expression in the unfed insect, and is again increased at 8 and 24 hours post-feeding. These differences in expression, however, are not statistically significant (two-way ANOVA, p>0.05). Expression of the Rhopr-CRF/DH transcript in the brain remains constant from pre- to post-feed. Error bars represent standard error of the means from three trials. 
$\square$ Brain
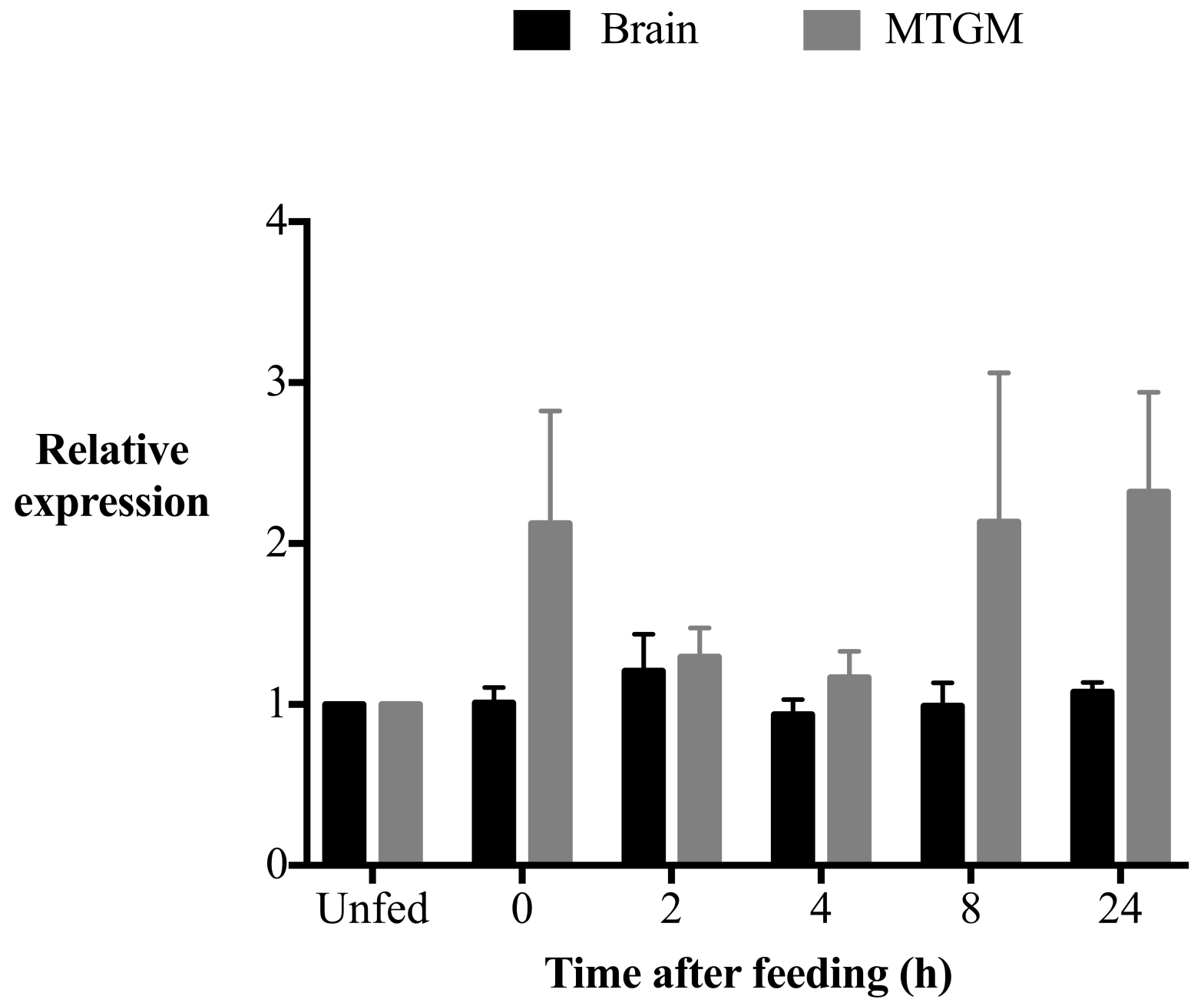


\subsection{Feeding assays}

Fifth instar $R$. prolixus, injected with1 $\mu \mathrm{L}$ of $10^{-4} \mathrm{M}$ Rhopr-CRF/DH 24 hours prior to feeding, consumed a smaller blood meal as evidenced by significantly lower post-feed weight relative to saline-injected controls (Fig. 6A). When insects were injected 2-3 hours prior to feeding, the inhibitory effect was more pronounced, with Rhopr-CRF/DH-injected insects weighing significantly less than controls after the blood meal (Fig. 6B). The same effect was observed in adult $R$. prolixus with injection of $2 \mu \mathrm{L}$ of $10^{-4} \mathrm{M}$ Rhopr-CRF/DH 2-3 hours prior to feeding (Fig. 7). There appeared to be no difference in feeding duration between treatment and control groups. Insects fed in groups of 20-25 individuals for just over 18 minutes, at which point feeding was terminated when insects removed their proboscises from the membrane and started moving about in their jars. The size of the blood meal taken by control adults is less than that of $5^{\text {th }}$ instars. Adults normally experience a three-fold increase in weight post-feeding, as opposed to the ten-fold increase in $5^{\text {th }}$ instars (Beckel and Friend, 1964). The rate of weight loss, a measure of diuresis, did not differ between saline- and Rhopr-CRF/DH injected insects (Figure $8 \mathrm{~A}, 8 \mathrm{~B})$.

Given that Rhopr-CRF/DH and serotonin act additively to increase Malpighian tubule secretion, we injected $5^{\text {th }}$ instars with $1 \mu \mathrm{L}$ of $10^{-4} \mathrm{M}$ serotonin 2-3 hours prior to feeding and found it had no effect on size of blood meal taken or rate of post-prandial weight loss, relative to salineinjected controls (Fig. 9). 
Figure 6. Feeding assay performed on $5^{\text {th }}$ instar $R$. prolixus.

(A) Insects injected with Rhopr-CRF/DH 24 hours prior to feeding consumed a significantly smaller blood meal compared to saline-injected controls, as depicted by post-feed body weights (Student's t-test, $* * * \mathrm{p} \leq 0.001$ ). (B) Insects injected with Rhopr-CRF/DH 2 - 3 hours before feeding showed a more pronounced decrease in blood intake relative to controls than did the insects injected 24 hours prior to feeding (Student's t-test, $* * * p=0.001$ ). Error bars represent standard error of the mean. 
A

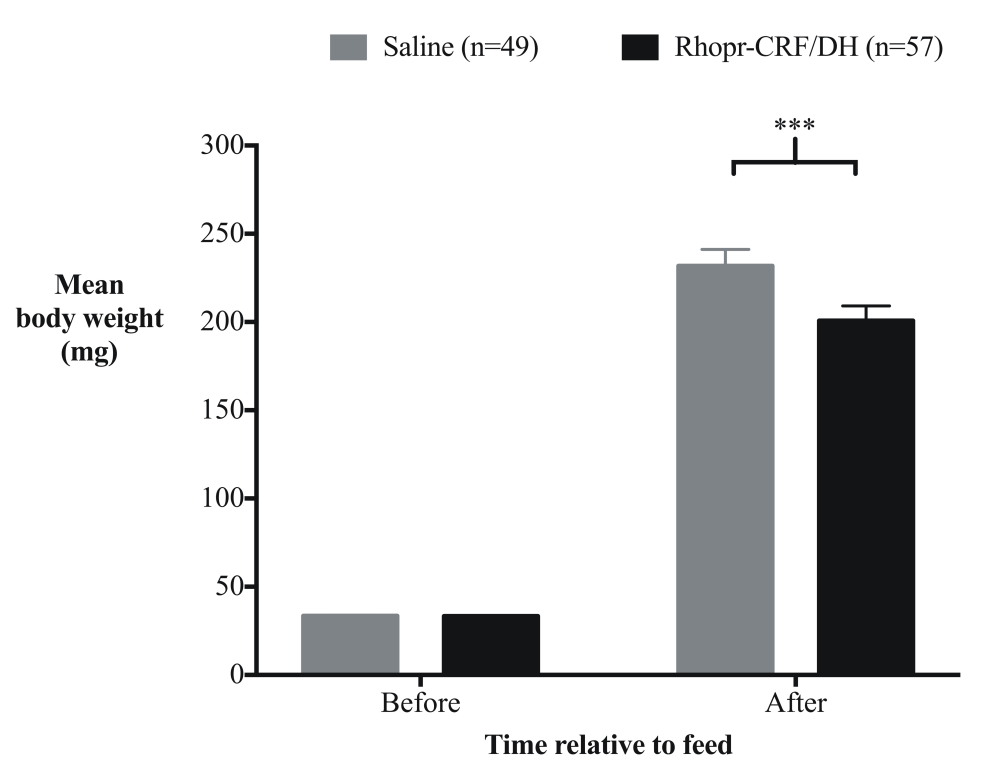

\section{B}

Saline (n=24) $\square$ Rhopr-CRF/DH (n=24)

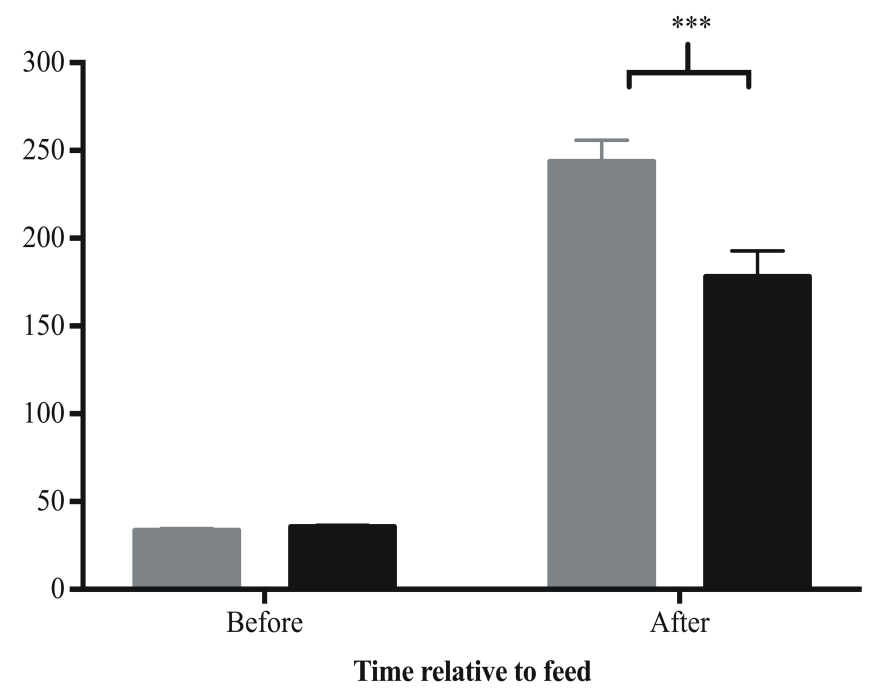


Figure 7. Feeding assay performed on adult $R$. prolixus.

Insects injected with Rhopr-CRF/DH 2-3 hours prior to feeding consumed a significantly smaller blood meal compared to saline-injected controls, as depicted by post-feed body weights (Student's t-test, $* * * \mathrm{p} \leq 0.001$ ). Error bars represent standard error of the mean. 


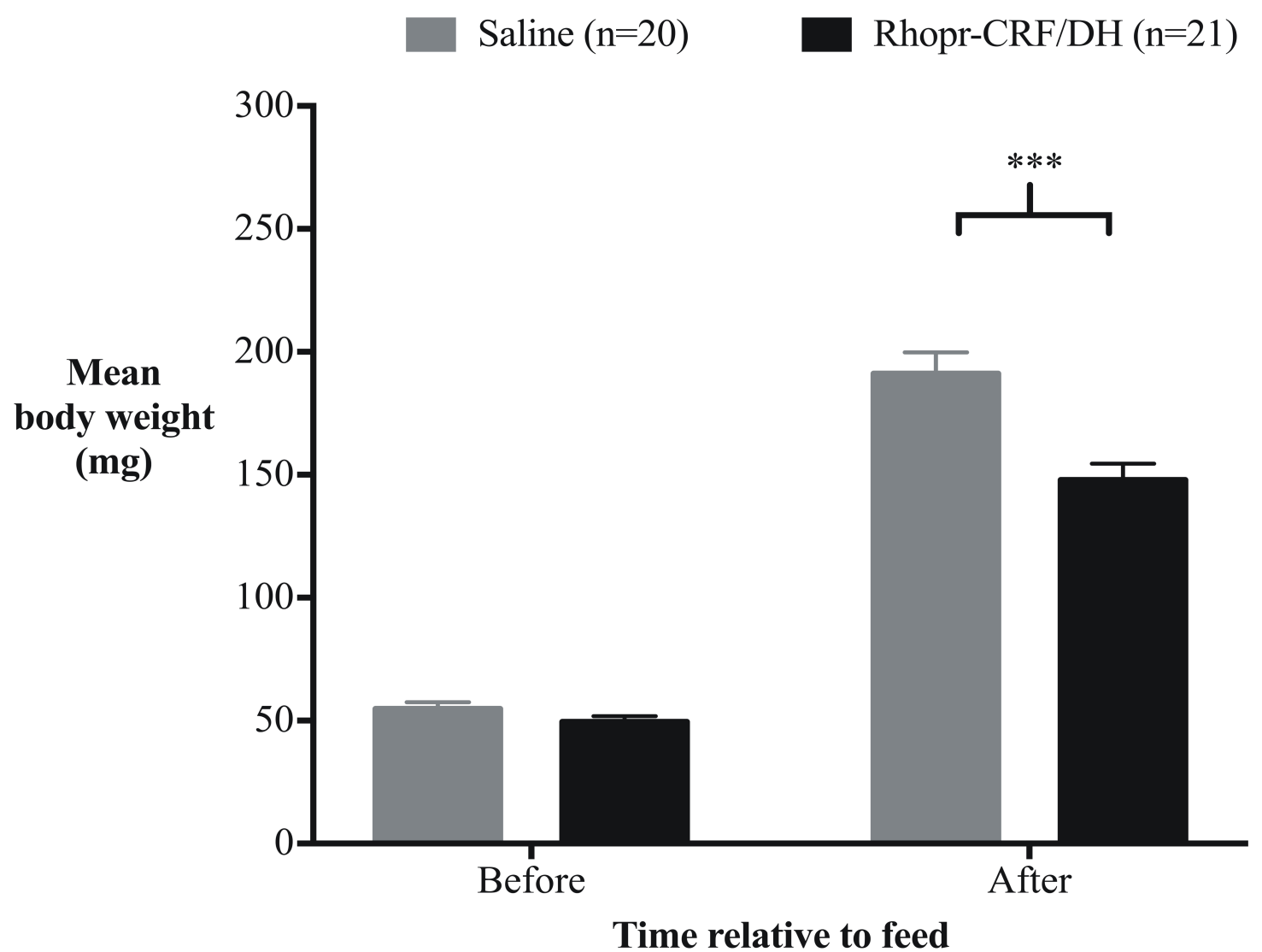


Figure 8. Weights of saline- and Rhopr-CRF/DH-injected insects (injection 2-3 hours prior to feeding) over the course of 4 hours after feeding to depict diuresis.

Rates of weight loss did not differ significantly between treatment and control groups in both $5^{\text {th }}$ instars (A) and adults (B), as determined by fitting the data using linear regression and analyzing the slopes for significance using an F-test $(\mathrm{p}>0.05)$. 
A

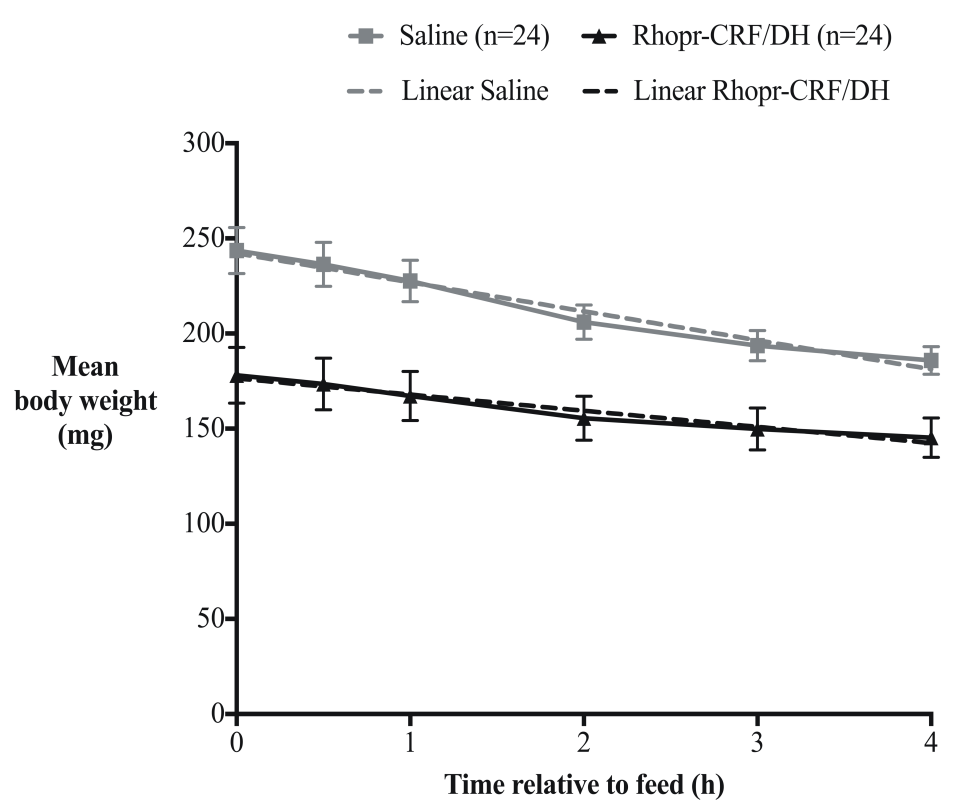

B
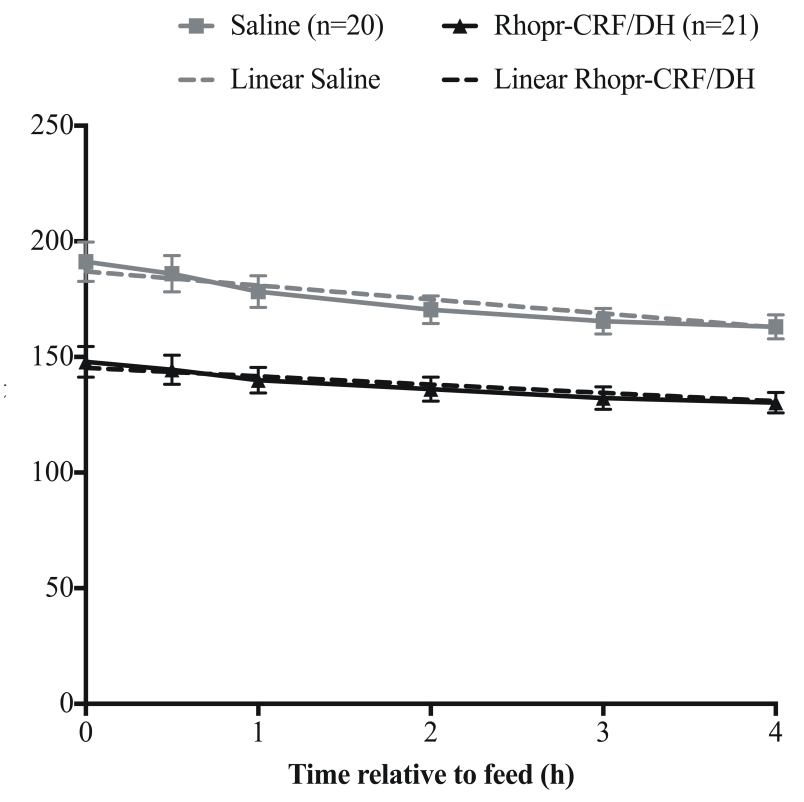
Figure 9. Feeding assay performed on $5^{\text {th }}$ instar $R$. prolixus.

Insects injected with 5-HT 2-3 hours prior to feeding did not differ from saline-injected controls in size of blood meal consumed (Student's t-test, $\mathrm{p}>0.05$ ) 


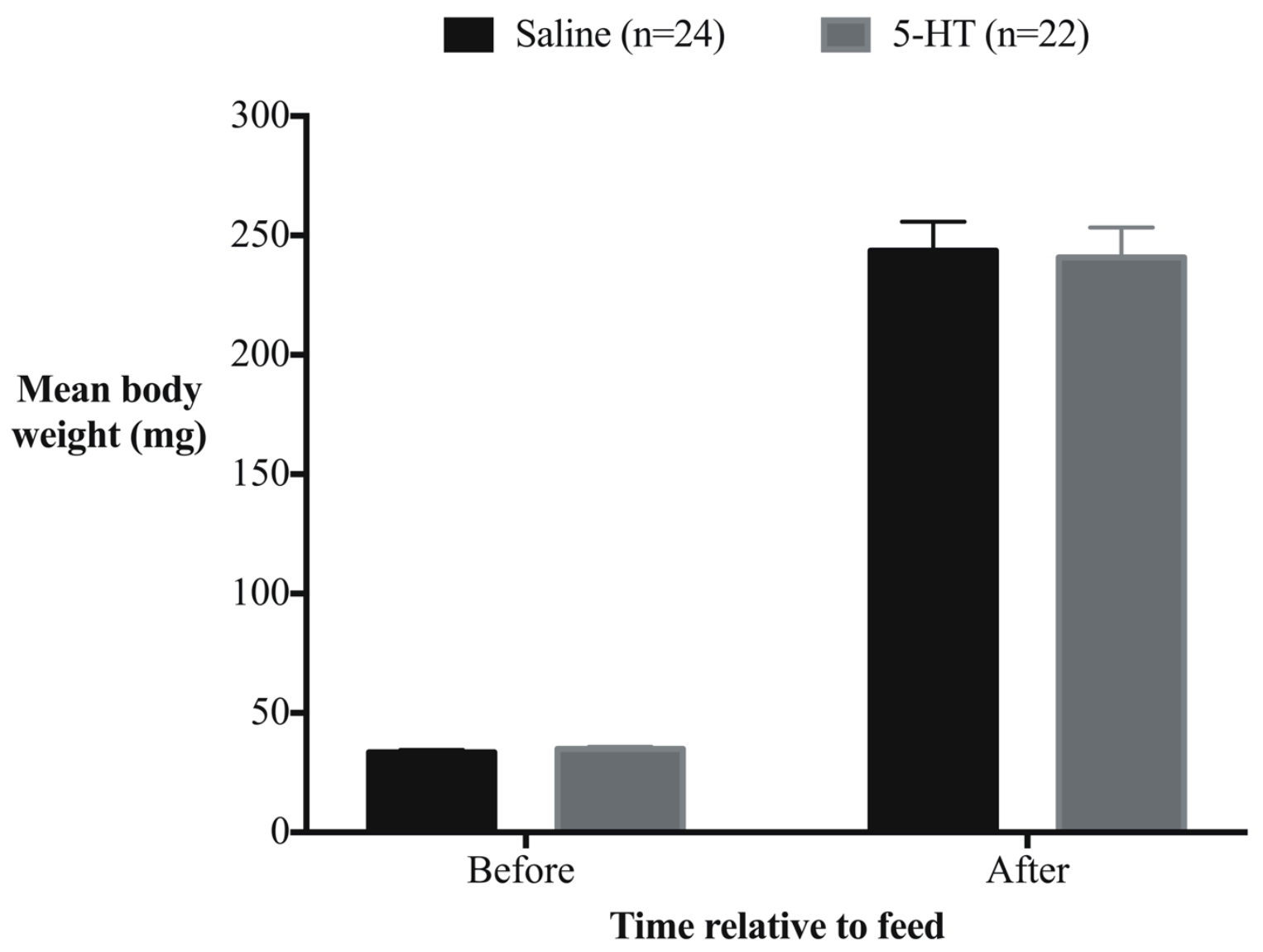




\subsection{Egg-laying assays}

Adult females injected with $2 \mu \mathrm{L}$ of $10^{-3} \mathrm{M}$ Rhopr-CRF/DH after mating generally laid fewer eggs daily over the course of 8 days compared to their saline-injected counterparts (Fig. 10A). Furthermore, the rate of egg laying appeared to plateau at 7 days after injection, while an upward trajectory remained for saline-injected controls (Fig. 10B). While these daily differences were not statistically significant, we did observe a significant difference in cumulative egg production and egg laying between treatment and control groups (Fig. 11). Rhopr-CRF/DH-injected females both made and laid significantly fewer eggs than did controls.

\subsection{Contraction assays}

Rhopr-CRF/DH had an inhibitory effect on in vitro oviduct contractions, as established by the decrease in amplitude of contractions, relative to saline (Fig. 12). The effects of Rhopr-CRF/DH were dose-dependent, with higher doses resulting in greater inhibition. Concentrations below $10^{-}$

${ }^{11} \mathrm{M}$ caused a slight but not statistical decrease in amplitude. At concentrations of $10^{-11} \mathrm{M}$ and higher, there was a significant decrease in amplitude of contractions relative to saline. The duration of inhibition by Rhopr-CRF/DH lasted for less than 100 seconds at all concentrations, before contractions resembling those observed when the oviducts were in saline resumed. 


\section{Figure 10. Results of egg-laying assay}

(A) Time course of egg-laying by adult female $R$. prolixus over a period of eight days after injection of Rhopr-CRF/DH. Adult females injected with Rhopr-CRF/DH generally laid fewer eggs per day than did saline-injected controls. These daily differences in egg laying were not significant (two-way ANOVA, $\mathrm{p}>0.05$ ). (B) Mean cumulative number of eggs per female per day. Saline- and Rhopr-CRF/DH-injected controls did not differ significantly in the number of eggs laid daily ( $p>0.05$ ), however, egg-laying in the Rhopr-CRF/DH group stars to plateau early, while controls are still experiencing an upward trajectory. 
A

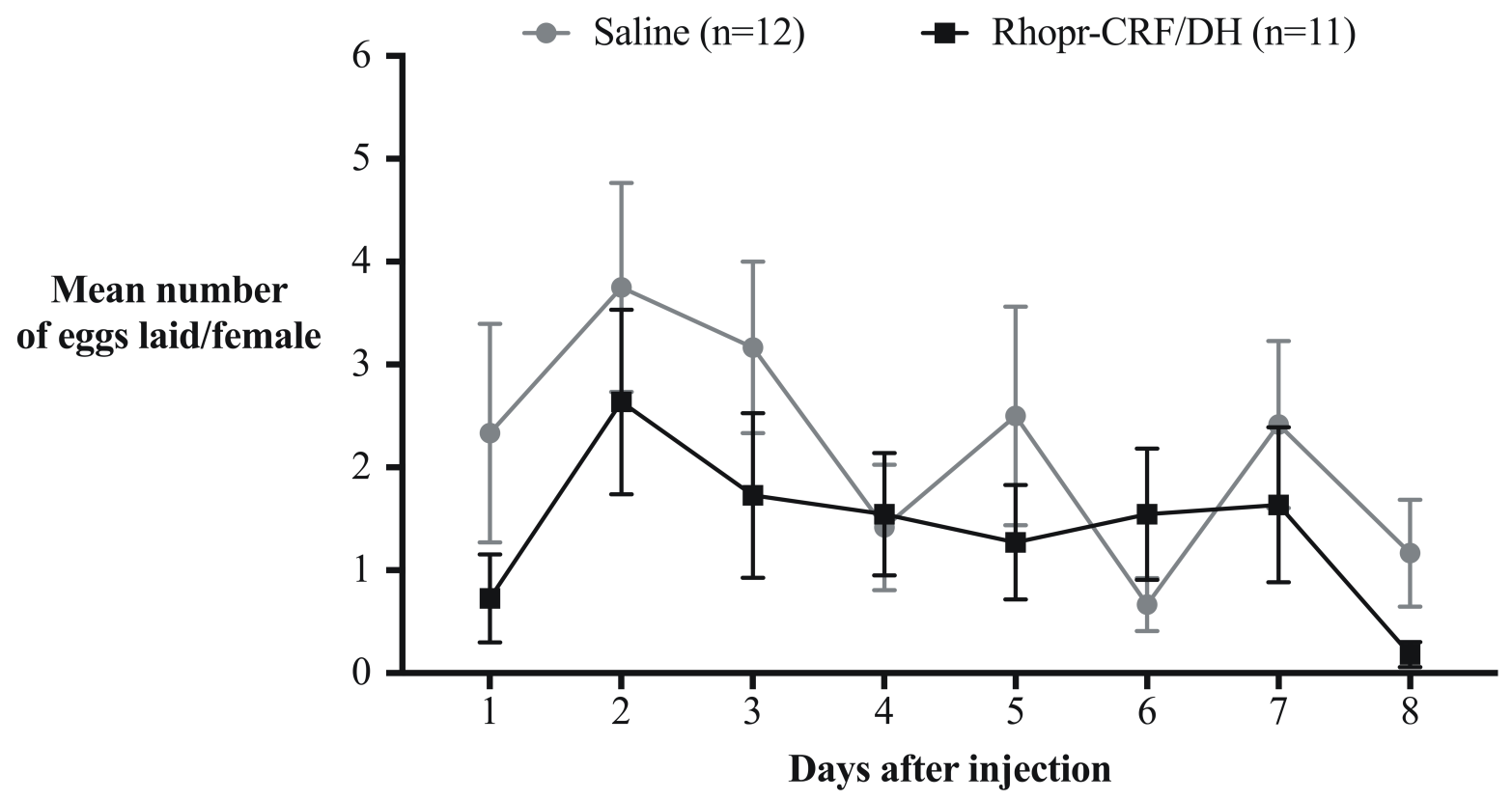

B

$\rightarrow$ Saline $(\mathrm{n}=12)$

$\rightarrow$ Rhopr-CRF/DH (n=11)

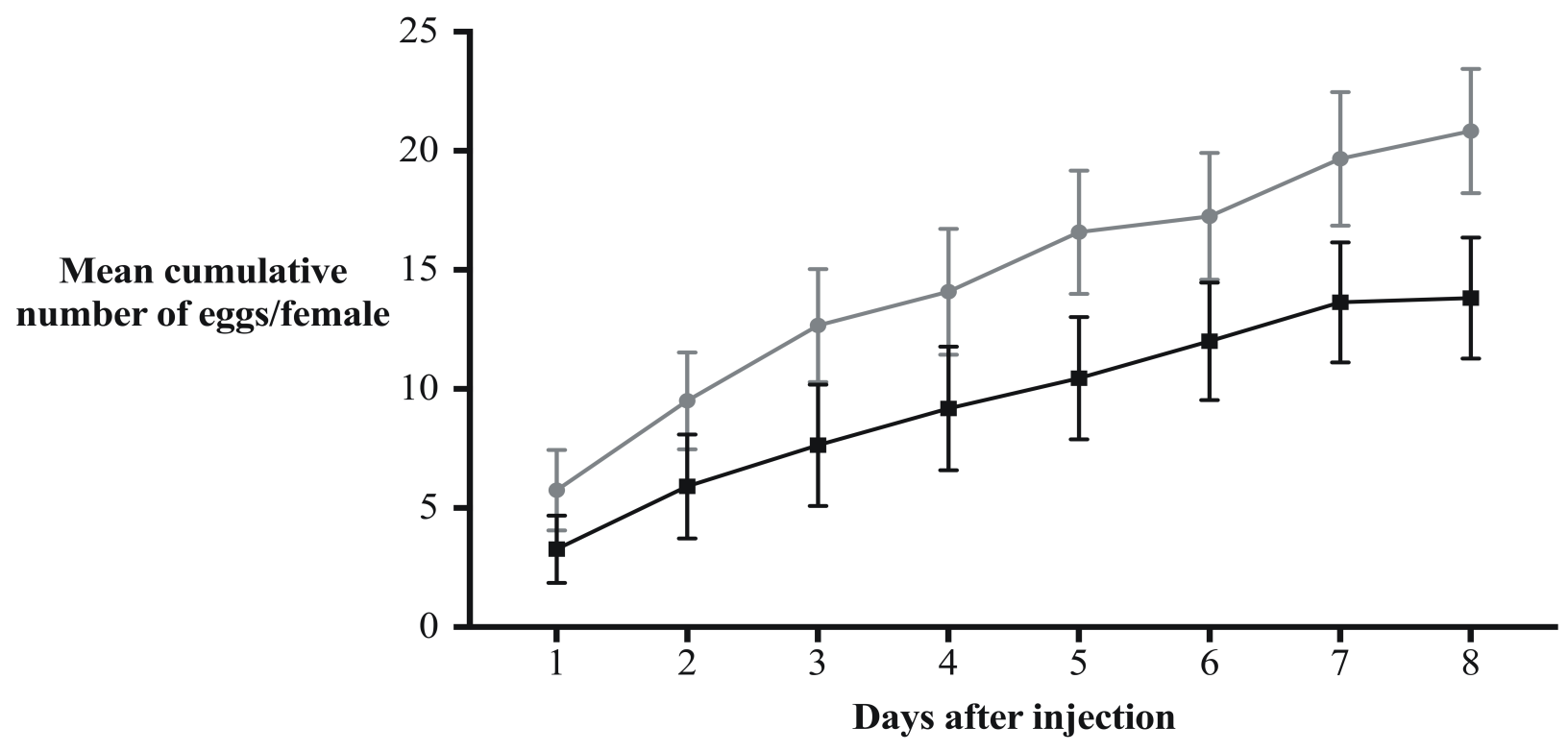




\section{Figure 11. Egg-laying assay cumulative results.}

Females injected with Rhopr-CRF/DH both produced and laid significantly fewer eggs over the course of eight days than those injected with saline (two-way ANOVA, ${ }^{*} \mathrm{p} \leq 0.05$ ). While more eggs were found in the ovaries and oviducts of the treatment females on the eighth day, these differences were not significant (two-way ANOVA, $\mathrm{p}>0.05$ ). Error bars represent standard error of the mean. 


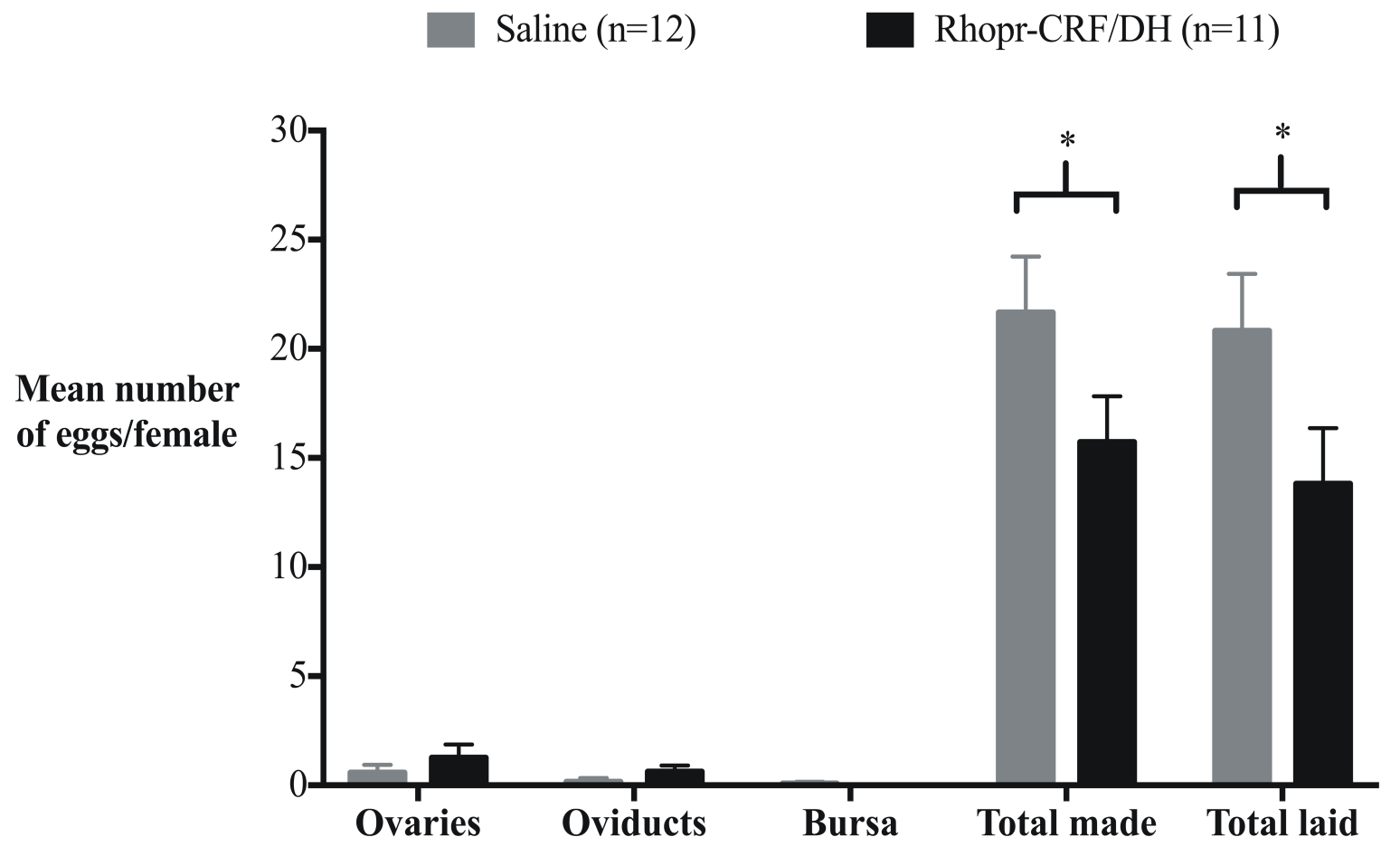


Figure 12. Results of in vitro oviduct contraction assay.

(A) Dose-response curve depicting the dose-dependent inhibitory effect of Rhopr-CRF/DH on spontaneous oviduct contractions. Burst amplitude is shown as a percentage relative to saline (100\%). Application of doses between $10^{-11} \mathrm{M}$ and $10^{-9} \mathrm{M}$ resulted in a significant decrease in

burst amplitude relative to saline (two-way ANOVA, ${ }^{*} \mathrm{p} \leq 0.05$ for $10^{-11} \mathrm{M}$ and ${ }^{* *} \mathrm{p} \leq 0.01$ for $10^{-10}$ $\mathrm{M}$ and $10^{-9} \mathrm{M}$ ). Error bars represent standard error of the mean of 3-5 replicates. (B) Physiological traces showing decreased burst amplitude (i.e., force of contractions) following application of different doses of Rhopr-CRF/DH. Arrow indicates point at which dose was applied; bursts preceding the arrow show contractions in saline. 
A

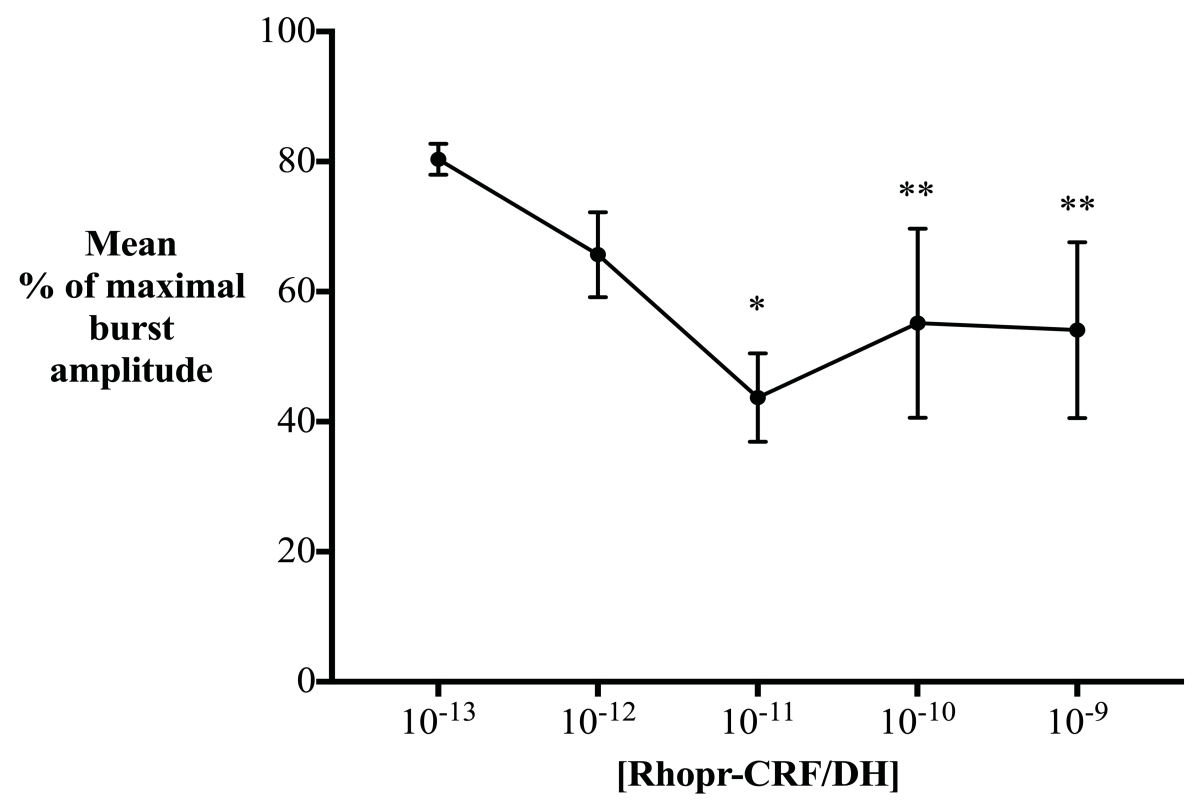

B
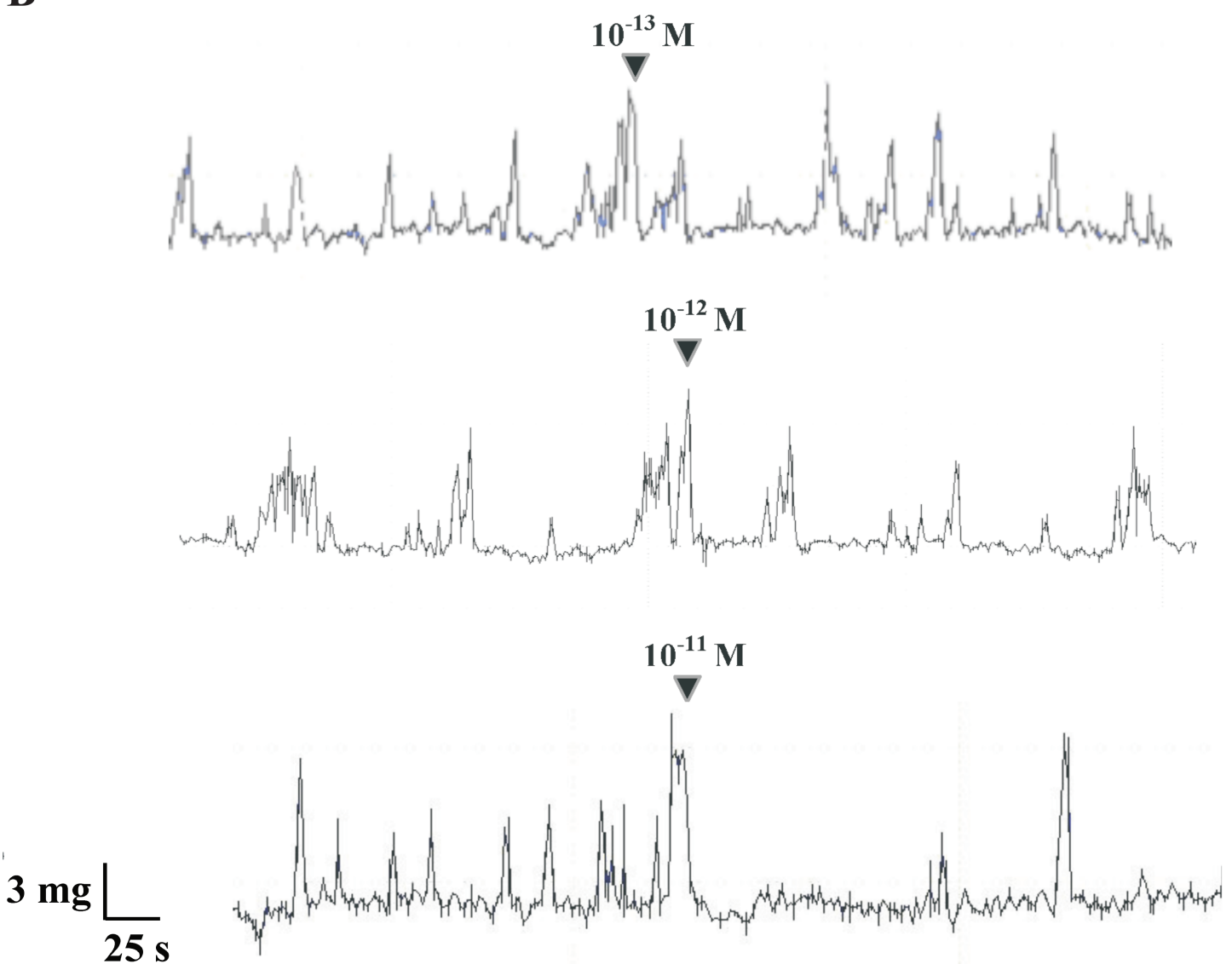


\section{Discussion}

The aim of this research study was to investigate the role of Rhopr-CRF/DH, a known diuretic hormone, in feeding and reproduction in R. prolixus. We found that the hormone acts in an inhibitory manner in both functions while stimulating diuresis. Insects injected with Rhopr$\mathrm{CRF} / \mathrm{DH}$ took in a significantly smaller blood meal; likewise, adult females injected with RhoprCRF/DH both made and laid significantly fewer eggs. The results support the notion that Rhopr$\mathrm{CRF} / \mathrm{DH}$ is a multifunctional hormone in $R$. prolixus.

Rhopr-CRF/DH exerts its effects through G-protein coupled receptors (GPCRs). A variant of one of two Rhopr-CRF/DH receptors (Rhopr-CRF/DH-Rs) has been isolated and sequenced in $R$. prolixus (Rhopr-CRF/DH-R2B) (Lee et al., 2016). It is a class B1 GPCR, which signals through the cyclic AMP (cAMP)-dependent pathway upon activation by Rhopr-CRF/DH (Lee et al., 2016). The molecular and physiological endpoints of this pathway in $R$. prolixus are not fully known, but research in vertebrates has described class B1 GPCRs as regulators of a variety of physiological processes, including feeding, metabolism and growth, diuresis, and reproduction (Grammatopoulos, 2012). Rhopr-CRF/DH-R2B is expressed in most systems in R. prolixus, with highest expression observed in parts of the reproductive, digestive, and excretory systems, highlighting the Rhopr-CRF/DH pathway as one potentially involved in more than one of the aforementioned processes (Lee et al., 2016).

CRF-like immunostaining has previously been shown to diminish in the lateral NSCs in the MTGM 1.5 hours after feeding and staining was restored at 24 hours (Te Brugge and Orchard, 2002). Here, we show that staining in the lateral NSC bodies in the MTGM and in neuronal processes along the abdominal nerves is significantly diminished immediately after feeding and increases two hours later, suggesting its release and restocking is associated with the process of feeding. The increase in Rhopr-CRF/DH transcript expression immediately after feeding is consistent with the observed diminishment of CRF-like immunostaining in the lateral NSCs of the MTGM, suggesting transcription of the Rhopr-CRF/DH gene is increased after feeding to restock the cells. The high variation in expression observed between replicates, and therefore the non-statistically significant changes in expression, in particular immediately after feeding, and at 8 and 24 hours could be highlighting critical points for expression that are dependent on the size of the meal taken by the insects (i.e., differences in feed intake related to differences in Rhopr- 
$\mathrm{CRF} / \mathrm{DH}$ release, which determined degree of restocking and thereby expression). The link between Rhopr-CRF/DH release from lateral NSCs in the MTGM and feeding is confirmed in our feeding assays, whereby increasing the titre of Rhopr-CRF/DH in the haemolymph by injection decreased blood meal intake. Our results demonstrating the inhibitory effect of Rhopr$\mathrm{CRF} / \mathrm{DH}$ on the feeding process in R. prolixus are consistent with what has previously been described in S. gregaria (van Wielendaele et al., 2012) and L. migratoria (Goldsworthy et al., 1999). In L. migratoria, the injection of Locmi-CRF/DH increased the latency to feed and decreased feeding duration (Goldsworthy et al., 1999). Injection of S. gregaria with Schgr$\mathrm{CRF} / \mathrm{DH}$ prior to feeding likewise resulted in a significantly reduced food intake (van Wielendaele et al., 2012). Audsley et al. (1997) showed that the Locmi-CRF/DH titers in the haemolymph increased 5-fold after feeding relative to starved insects, and the level of circulating hormone was correlated with time spent feeding, and so presumably with the size of the meal. In R. prolixus, Rhopr-CRF/DH would appear to provide a feedback signal to terminate feeding, not dependent upon the process of diuresis, as its injection 2-3 hours prior to feeding did not alter the rate of diuresis. Moreover, serotonin, a diuretic that acts additively with Rhopr-CRF/DH on the Malpighian tubules to stimulate secretion (Te Brugge et al., 2002), was not found to have an effect on feeding. Thus, while Rhopr-CRF/DH is classified as a diuretic hormone, its release and restocking in the lateral NSCs in the MTGM during and after a blood meal appears to be a stimulus not only for diuresis, but also for the termination of feeding.

CRF's involvement in the stress response in vertebrates (Yau and Potenza, 2013), which, among other behavioural manifestations results in reduced food intake, correlated with the feeding inhibition effects we observed in R. prolixus. In mice, injection of CRF resulted in significantly decreased food intake (Krahn et al., 1986), a response that was also observed with application of restraint stress (Jeong et al., 2013); in rats, Ucn-2, a peptide belonging to the CRF family, was found to reduce meal size and rate of ingestion (Fekete et al., 2011). Mammalian CRF activates the hypothalamic-pituitary-adrenal axis, stimulating hormone secretion to maintain homeostasis (Smith and Vale, 2006). In insects, the neuroendocrine system also consists of NSCs and endocrine cells, and glands distributed throughout the CNS and peripheral tissues (Hartenstein, 2006). They produce hormones that are released into the haemolymph and bind to receptors on target tissues. Thus, it is plausible that, just as in vertebrates, endogenous Rhopr-CRF/DH acts as a stress response hormone in $R$. prolixus, terminating feeding once the insect has reached a stress 
threshold during blood-gorging as a result of distension of the abdomen, internal pressure changes, and/or osmotic challenge, and possibly signals the release of other feeding-regulating chemical messengers to maintain homeostasis. The Rhopr-CRF/DH NSCs in the brain are apparently not necessary to control the rapid post-feeding diuresis (Maddrell, 1964), but do show reduced staining intensity following feeding, and so may also participate in other aspects of feeding, including satiety or reproduction. Interestingly, we did not observe a difference in feeding duration after injection of Rhopr-CRF/DH indicating that the decreased blood meal intake can be attributed to a reduction in rate of feeding induced by Rhopr-CRF/DH. The mechanism underlying this effect is not known. Gäde and Goldsworthy (2003) attributed the CRF's satiety-inducing effects to the decrease in peripheral sensitivity to food in L. migratoria, showing that Locmi-CRF/DH caused closure of the pores on the taste sensilla, impeding the insects' sense of taste. A similar mechanism may be at play in $R$. prolixus.

Spatial expression analysis of Rhopr-CRF/DH-R2B provides insight into other physiological functions of Rhopr-CRF/DH (Lee et al., 2016). The highest expression of the receptor was observed in the male immature testes, the foregut and female immature ovaries in $5^{\text {th }}$ instar $R$. prolixus. Interestingly, when expression was compared between the reproductive systems of $5^{\text {th }}$ instars and adults, there was a significant increase in expression of the receptor in the mature ovaries relative to the immature ovaries of $5^{\text {th }}$ instars (Lee et al., 2016).

Injection of Rhopr-CRF/DH into mated female $R$. prolixus resulted in a significant reduction in the number of eggs produced and laid. A similar effect was observed in S. gregaria, where injection of Schgr-CRF/DH was found to impede oocyte growth and to reduce levels of ecdysteroids in the haemolymph (van Wielendaele et al., 2012). Most of the research on the effects of CRF on reproduction has been done in mammals; nevertheless, the findings in insects are consistent with what has been previously depicted in mammals. The CRF receptor is present in the ovaries of rats and humans, and CRF itself has been implicated in stress-induced reproductive inhibition (Vitoratos et al., 2006). CRF has been shown to inhibit steroid biosynthesis, with injection of the hormone into rats attenuating release of gonadotropinreleasing hormone and luteinizing hormone, which trigger ovulation and steroidogenesis (Kageyama, 2013). If, as we hypothesize, Rhopr-CRF/DH is a stress hormone in R. prolixus, then it is possible its inhibitory effects with respect to egg production and egg-laying are caused by the feeding-induced increase in Rhopr-CRF/DH haemolymph titre, activating the stress 
response in the insect and signalling the delay of energy-consuming processes such as reproduction. A possible mechanism by which egg laying is inhibited in females injected with Rhopr-CRF/DH is through inhibition of muscle contractions of the oviduct. The results of our lateral oviduct contraction assays demonstrate a significant decrease in the amplitude of

spontaneous oviduct contractions with application of Rhopr-CRF/DH doses of $10^{-11} \mathrm{M}$ to $10^{-9} \mathrm{M}$.

Our egg-laying assays showed an increased number of eggs in the ovaries and oviducts of treatment insects after 8 days post-injection. Although these differences were not statistically significant, it is possible that injected Rhopr-CRF/DH binds to receptors on the oviducts and inhibits contractions, thereby impeding the eggs' movement into the bursa and successful release. Therefore, Rhopr-CRF/DH may be acting on reproduction by two different mechanisms; firstly, by altering the neuroendocrine control of egg growth and development leading to fewer eggs being produced and secondly, by altering the contractility of oviduct muscle and thereby disrupting egg deposition.

The present work shows, for the first time, that Rhopr-CRF/DH alters feeding, and egg production and egg laying in $R$. prolixus. The mechanisms by which Rhopr-CRF/DH exerts these effects are yet to be fully investigated. Future work utilizing molecular techniques to knockdown Rhopr-CRF/DH and its receptor and observe the effects on physiology will be of particular interest. Understanding the Rhopr-CRF/DH pathway, its components and mechanisms of action, has implications for vector control by highlighting targets to alter feeding, diuresis, and reproduction of this disease vector.

\section{Acknowledgements}

This work was supported by Natural Sciences and Engineering Research Council of Canada Discovery Grants to IO and ABL. We would like to acknowledge Merima Vila for maintaining the $R$. prolixus colony and for fulfilling last-minute feeding requests. 


\section{References}

Audsley, A., Goldsworthy, G.J., Coast, G.M. 1997. Circulating levels of Locusta diuretic hormone: the effect of feeding. Peptides. 18, 59-65. doi: 10.1016/S0196-9781(96)00234-3

Beckel, W.E., Friend, W.G. 1964. The relation of abdominal distension and nutrition to molting in Rhodnius prolixus (Stahl) (Hemiptera). Can. J. Zool. 42, 71-78. doi: 10.1139/z64-007

Centers for Disease Control and Prevention, 2017. Chagas disease - detailed FAQs. https://www.cdc.gov/parasites/chagas/gen_info/detailed.html (accessed 17/03/01).

Coast, G.M., Orchard, I., Phillips, J.E., Schooley, D.A. 2002. Insect diuretic and antidiuretic hormones. Adv. Insect Physiol. 29, 279-409. doi: 10.1016/S0065-2806(02)29004-9

Fekete, E.M., Zhao, Y., Szucs, A., Sabino, V., Cottone, P., Rivier, J., Vale, W.W., Koob, G.F., Zorrilla, E.P., 2011. Systemic urocortin 2, but not urocortin 1 or stressin 1-A, suppresses feeding via CRF 2 receptors without malaise and stress. Br. J. Pharmacol. 164, 1959-1975.

doi:10.1111/j.1476-5381.2011.01512.x

Ferreira, T., Rasband, W. 2012. ImageJ user guide. https://imagej.nih.gov/ij/docs/guide/userguide.pdf (accessed 17/03/01)

Gäde, G., Goldsworthy, G.J., 2003. Insect peptide hormones: A selective review of their physiology and potential application for pest control. Pest Manag. Sci. doi:10.1002/ps.755

Girardie, J., Geoffre, S., Delbecque, J.P., Girardie, A., 1998. Arguments for two distinct gonadotropic activities triggered by different domains of the ovary maturating parsin of Locusta migratoria. J. Insect Physiol. 44, 1063-1071. doi: 10.1016/S0022-1910(98)00066-3

Goldsworthy, G.J., Owusu, M., Ross, K.T.A., Simmonds, M.S.J., Coast, G.M., et al. 1999. Locusta CRF-related diuretic peptide is a factor mediating satiety in locusts. In: Roubos E.W., Wendelaar Bonga S.E., Vaundry, H., De Loof, A. (Eds.) Recent developments in comparative endocrinology and neurobiology. Shaker Publishing BV, Maastricht, pp. 231-234.

Grammatopoulos, D.K., 2012. Insights into mechanisms of corticotropin-releasing hormone receptor signal transduction. Br. J. Pharmacol. doi: 10.1111/j.1476-5381.2011.01631.x

Hartenstein, V., 2006. The neuroendocrine system of invertebrates: A developmental and evolutionary perspective. J. Endocrinol. doi: 10.1677/joe.1.06964

Jeong, J.Y., Lee, D.H., Kang, S.S., 2013. Effects of chronic restraint stress on body weight, food intake, and hypothalamic gene expressions in mice. Endocrinol. Metab. (Seoul, Korea) 28, 28896. doi: 10.3803/EnM.2013.28.4.288

Kageyama, K., 2013. Regulation of gonadotropins by corticotropin-releasing factor and urocortin. Front. Endocrinol. (Lausanne). doi: 10.3389/fendo.2013.00012 
Krahn, D.D., Gosnell, B.A., Grace, M., Levine, A.S., 1986. CRF antagonist partially reverses CRF- and stress-induced effects on feeding. Brain Res. Bull. 17, 285-289. doi: 10.1016/03619230(86)90233-9

Lee, H.R., Zandawala, M., Lange, A.B., Orchard, I. 2016. Isolation and characterization of the corticotropin-releasing factor-related diuretic hormone receptor in Rhodnius prolixus. Cell Signal. 28, 1152-1162. doi: 10.1016/i.cellsig.2016.05.020

Maddrell, S.H., 1964. Excretion in the Blood-Sucking Bug, Rhodnius Prolixus Stal. 3. the Control of the Release of the Diuretic Hormone. J. Exp. Biol. 41, 459-472.

Majzoub, J. a, 2006. Corticotropin-releasing hormone physiology. Eur. J. Endocrinol. 155, S71S76. doi: 10.1530/eje.1.02247

Martini, S. V., Nascimento, S.B., Morales, M.M., 2007. Rhodnius prolixus Malpighian tubules and control of diuresis by neurohormones. An. Acad. Bras. Cienc. 79, 87-95. doi: 10.1590/S0001-37652007000100011

Orchard, I. 2006. Serotonin: a coordinator of feeding-related physiological events in the bloodgorging bug, Rhodnius prolixus. Comp. Biochem. Physiol. 144A, 316-324. doi:

10.1016/j.cbpa.2005.11.010

Orchard, I. 2009. Peptides and serotonin control feeding-related events in Rhodnius prolixus. Front. Biosci. (Elite Ed.) 1, 250-262.

Sedra, L., Haddad, A.S., Lange, A.B., 2015. Myoinhibitors controlling oviduct contraction within the female blood-gorging insect, Rhodnius prolixus. Gen. Comp. Endocrinol. 211, 62-68. doi: 10.1016/j.ygcen.2014.11.019

Smith, S.M., Vale, W.W., 2006. The role of the hypothalamic-pituitary-adrenal axis in neuroendocrine responses to stress. Dialogues Clin Neurosci 8, 383-395. doi:

10.1038/nrendo.2011.222

Te Brugge, V.A., Miksys, S.M., Coast, G.M., Schooley, D.A., Orchard, I. 1999. The distribution of a CRF-like diuretic peptide in the blood-feeding bug Rhodnius prolixus. J. Exp. Biol. 202, 2017-27.

Te Brugge, V.A., Nässel, D.R., Coast, G.M., Schooley, D.A., Orchard, I., 2001. The distribution of a kinin-like peptide and its co-localization with a CRF-like peptide in the blood-feeding bug, Rhodnius prolixus. Peptides 22, 161-173. doi: 10.1016/S0196-9781(00)00373-9

Te Brugge, V.A., Orchard, I. 2002. Evidence for CRF-like and kinin-like peptides as neurohormones in the blood-feeding bug, Rhodnius prolixus. Peptides 23, 1976-1979. doi: 10.1016/S0196-9781(02)00184-5

Te Brugge, V.A., Schooley, D.A., Orchard, I. 2002. The biological activity of diuretic factors in Rhodnius prolixus. Peptides 23, 671-681. doi: 10.1016/S0196-9781(01)00661-1 
van Wielendaele, P., Dillen, S., Marchal, E., Badisco, L., Vanden Broeck, J., 2012. CRF-like diuretic hormone negatively affects both feeding and reproduction in the desert locust, Schistocerca gregaria. PLoS One 7. doi: 10.1371/journal.pone.0031425

Vitoratos, N., Papatheodorou, D.C., Kalantaridou, S.N., Mastorakos, G., 2006. "Reproductive" corticotropin-releasing hormone, in: Annals of the New York Academy of Sciences. pp. 310318. doi: 10.1196/annals.1365.029

World Health Organization, 2017. Chagas disease.

http://www.who.int/mediacentre/factsheets/fs340/en/ (accessed 17/03/01).

Yau, Y.H.C., Potenza, M.N., 2013. Stress and eating behaviors. Minerva Endocrinol. doi: 10.3410/B2-13 


\section{Chapter 3 \\ RNAi: Silencing Rhopr-CRF/DH and its receptor}

\section{Introduction}

To make a more definitive conclusion about the role of Rhopr-CRF/DH signalling in feeding and reproduction, synthesis of double-stranded RNA (dsRNA) targeting the peptide and receptor transcripts, to induce RNA interference (RNAi), followed. By reducing or eliminating expression of one or both of these constructs and performing feeding and egg-laying assays, one can get insight into the significance of their roles in the respective processes. Based on the results of these assays following peptide injection (see Chapter 2), it is expected that feeding will proceed indefinitely in the absence of a termination signal by Rhopr-CRF/DH. The effect of RNAi on reproduction, on the other hand, depends on the mechanism by which Rhopr-CRF/DH alters reproductive processes - if it signals for the attenuation of these processes until sufficient nutrients are available, then it is expected that oogenesis and oviposition will proceed in the absence of this signal, and thus without sufficient nutrients to meet the energy demands, resulting in reduced quantity or quality of oocytes. If its mechanism of action is through inhibition of muscle contractions, then knockdown of the neurohormone is expected to result in increased egg laying. Finally, if Rhopr-CRF/DH binds to its receptors on the ovaries, interfering with the processes that take place there, such as uptake of material from the trophic core and vitellogenesis, then its knockdown would presumably prevent these effects and results in increased oogenesis, ovulation, and oviposition.

\section{Background}

\subsection{RNA interference: dsRNA processing}

RNAi is a form of posttranscriptional gene control that occurs in eukaryotic cells and is mediated by small non-coding RNA molecules. These include microRNAs (miRNAs) and short interfering RNAs (siRNAs), both of which are generated from dsRNA (Jinek and Doudna, 2009), and PIWI-interacting RNAs (piRNAs), which arise from transposable elements in the genome (Ishizu et al., 2012). Approximately 30\% of genes in the human genome are controlled by miRNAs (Lewis et al., 2005). Utilizing dsRNA with a sequence complementary to a gene of interest, together with the host organisms own cell machinery can be used to silence the gene in 
question (Burand and Hunter, 2012; Scott et al., 2013). The dsRNA introduced into a cell is processed by RNase Dicer, which cleaves the long molecule into shorter 20-25 bp fragments, producing miRNAs and siRNAs (Diedrichs and Haber, 2007; Scott et al., 2013). These small RNA molecules associate with RNA-induced silencing complexes (RISCs), ribonucleoproteins with a core protein from the Argonaute (Ago) family, which possess endonucleolytic activity (Cenik and Zamore, 2011) and are responsible for the ultimate degradation of target molecules. The guide strand of the small RNA directs the RISC to its complementary mRNA, where Ago breaks the phosphodiester bond between the target mRNA nucleotides paired with the $10^{\text {th }}$ and $11^{\text {th }}$ nucleotides of the small RNA (Cenik and Zamore, 2011). The RISC is loaded with dsRNA in a specific orientation with the help of ATP and chaperone proteins Hsc70 and Hsp90, which use the ATP energy source to allow the Ago to accommodate the dsRNA. The positioning of the dsRNA within the Ago (5' end in the phosphate-bindings domain of Ago), as well as the first nucleotide, are determinants of strand identity; guide, which RISC will use to locate and degrade the target mRNA, or passenger, which is rendered useless and subsequently degraded (Cenik and Zamore, 2011). The small guide RNA, containing the 'seed sequence', in complex with RISC, locates and binds to its target mRNA by base pairing in the region spanning nucleotides 2-7/8. The duplex is subsequently ligated and thus expression of a gene is repressed. Some Agos do not possess endonucleolytic activity and so regulate expression by other means, such as, directing the mRNA to specific locations in the cell where mRNA decay is known to take place, and interfering with translation (Cenik and Zamore, 2011).

\section{2 dsRNA uptake and impact}

The manner in which dsRNA is incorporated into an organism determines the subsequent extent of impact of RNAi on the system. RNAi can occur in a cell autonomous and non-cell autonomous manner (Huvenne and Smagghe, 2010). Cell autonomous RNAi involves introduction (or expression, in the case of endogenous RNAi) of dsRNA into an individual cell, and its effect (i.e., silencing) is therefore restricted to that one cell. In the case of non-cell autonomous RNAi, dsRNA is introduced to cells from outside its site of synthesis or expression, through environmental or systemic transfer. In environmental transfer, dsRNA is taken up from the environment by any number of cells. In systemic transfer, dsRNA is also taken up from the environment, by a single cell, and that cell then transports the dsRNA to other cells. Cell autonomous RNAi is the most extensively described mechanism of dsRNA uptake, involving its 
cleavage and amplification by Dicer into siRNAs, subsequent incorporation into RISC, binding with target mRNA and subsequent degradation of the specific mRNA (Huvenne and Smagghe, 2010). This is the process that takes places in the individual cell once dsRNA is introduced, whether by environmental or systemic means, or expressed, and so it is said that autonomous RNAi follows non-autonomous RNAi (Huvenne and Smagghe, 2010). The mechanism by which dsRNA is amplified in a cell, as well as the mechanism of cell-to-cell transfer of dsRNAs that results in a systemic effect in insects, are not yet fully understood. The current literature, nevertheless, highlights two distinct mechanisms for dsRNA entry into the cell, via transmembrane channels.

In Caenorhabditis elegans, transmembrane protein SID-1, found on the cell periphery, was found to be essential for systemic RNAi. SID-1 is thought to be responsible for passive transport of dsRNA into cells. C. elegans with mutations in the SID-1 gene did not have the systemic silencing normally observed following introduction of dsRNA (Winston et al., 2002). SID-2 has been implicated as a SID-1 helper protein, possibly involved in the delivery of dsRNA to or activation of SID-1, as well as uptake of dsRNA via endocytosis and release of the molecule into the cytoplasm by SID-1 (Jose and Hunter, 2007; Winston et al., 2007). Both SID-2 and SID-5 have been implicated in uptake of dsRNA by intestinal cells, as would occur if dsRNA was ingested, as well as exit of dsRNA from cells, suggesting it may be involved in cell-to-cell transfer and thereby systemic RNAi (Scott et al., 2013). SID-1 orthologs have been identified in other insects belonging to the Coleoptera, Hemiptera, Hymenoptera, Lepidoptera, Orthoptera, and Phtiraptera orders (Huvenne and Smagghe, 2010). In the honeybee Apis mellifera, expression of the SID-1 gene increased prior to knockdown of a gene of interest by RNAi, implying involvement in dsRNA uptake; however, in the red flour beetle Tribolium castaneum, no effect on RNAi was observed (Huvenne and Smagghe, 2010). In Rhodnius. prolixus, a SID-1 ortholog has not been found, suggesting an alternate pathway is responsible for induction of RNAi (Paim et al., 2013).

An alternate endocytosis-mediated pathway has been described in Drosophila. melanogaster, in whichom SID orthologs have also not been found. Twenty-three genes ofrom over 7000 were identified as having a role in environmental RNAi, specifically in vesicle formation (Huvenne and Smagghe, 2010). Among them was a gene coding for the clathrin heavy chain (Chc), known for its role in vesicle formation. A gene coding for vacuolar $\mathrm{H}^{+}$ATPase is thought to have a role 
in induction of RNAi in D. melanogaster, as deficient flies retained dsRNA in vesicles, and silencing was not observed. When orthologous genes related to endocytosis were silenced in $C$. elegans, the RNAi pathway was disrupted, suggesting the vesicle mediated uptake of dsRNA is a conserved mechanism (Huvenne and Smagghe, 2010). There are also scavenger receptors in $D$. melanogaster, Eater and SR-CI, which are involved in the phagocytosis of bacterial pathogens. Both were found to be important for dsRNA uptake, responsible for over $90 \%$ of the uptake observed in haemocyte-derived S2 cells (Huvenne and Smagghe, 2010).

\subsection{Methods of dsRNA delivery}

In insects, several methods of dsRNA delivery have been described, and their relative efficacies reported. The most common of these methods is injection into the haemolymph orand ingestion, which have been demonstrated in D. melanogaster, Aedes aegypti, Tribolium castaneum, Manduca sexta, Acyrthosiphon pisum, and Apis mellifera. In A. aegypti and A. mellifera, both methods showed high success with respect to silencing of the gene of interest. In T. castaneum, however, injection was ideal, and in D. melanogaster ingestion was superior to injection. Despite the inherent risk of mechanical damage that comes with injection, it has certain advantages over other methods, particularly feeding, as it can deliver the dsRNA to a specific region of interest at a known concentration, neither of which can be achieved with feeding. Introduction of dsRNA by injection has been demonstrated to be successful in R. prolixus (Lee et al., 2013; Paim et al., 2013). The target molecules in the study by Paim and colleagues were nitrophorins (NPs) and rhodniin, the former of which has a multitude of functions and is expressed in the salivary glands (SG), and the latter an inhibitor of the coagulation cascade (Paim et al., 2013). In $3^{\text {rd }}$ instar insects, 98\% knockdown was achieved $48 \mathrm{~h}$ after injection with dsRNA targeting NP2, though this was reflected in protein content in the SG only after the insects were fed and had ecdysed into $4^{\text {th }}$ instars. The effect persisted through all life stages, reaching a point of nearly complete silencing (99\% reduction in protein levels). Interestingly, when $5^{\text {th }}$ instars were injected with dsNP2, RNAi silencing was observed in the progeny, which showed an over $80 \%$ reduction in both transcript and protein levels (Paim et al., 2013). With respect to rhodniin, $48 \mathrm{~h}$ after injection of $3^{\text {rd }}$ instars with dsRNA, a $60 \%$ reduction in transcript levels was observed in the midgut. After feeding and ecdysis, $5^{\text {th }}$ instars demonstrated an over $90 \%$ reduction in transcript levels, which persisted through all life stages. The phenotypic effects of RNAi against rhodniin were apparent within the same life stage that injection of dsRNA occurred. A $68 \%$ reduction in 
coagulant activity was observed in the midgut of injected nymphs and this reduction became more pronounced after ecdysis and persisted for months (Paim et al., 2013). The described work implies that, despite the absence of SID homologs in R. prolixus, this model organism must employ other means of import and export of dsRNA, given that dsRNA injected into the haemolymph was able to induce silencing in the SG and midgut. Furthermore, this work demonstrates the suitability of injection for delivery of the RNAi trigger to R. prolixus, as well as the long-term persistence and effects of the signal, highlighting the potential of this technique as a route to be explored with respect to vector control.

Feeding insects on medium into which dsRNA has been incorporated has been highlighted as less invasive and a highly effective method for inducing RNAi. In the Colorado potato beetle Leptinotarsa decemlineata, orally-administrated dsRNA was successful in inducing RNAi, and delivery of dsRNA into the cell was found to involve both the channel-mediated SID-1 pathway, as well as the endocytic pathway (Cappelle et al., 2016). In RNAi experiments targeting amylase, when SID-1-like genes silA and silC were also silenced, the silencing of amylase was rescued, with treatment insects expressing amylase mRNA relative to those in whom SID-1 was not silenced. Likewise, when components of the endocytic pathway were silenced, specifically the vha16 transmembrane domain of the vacuolar $\mathrm{H}^{+}$ATPase complex, RNAi of amylase was rescued. When the gene coding for Che was knocked down, mortality and disturbed feeding, which would impact expression of digestive enzyme amylase, were observed and thus reliable analysis of the role of the endocytic pathway in RNAi in the insects was obstructed, as the effect of feeding could not be disentangled from the effect of Chc with respect to amylase expression. However, when expression of a gene unrelated to feeding, coding for oxidase enzyme laccase, was analyzed, some rescue was seen in its knockdown when treated with dsChc, and a significant rescue was observed in treatment with endocytosis inhibitor chlorpromazine hydrochloride (Cappelle et al., 2016).

While both mechanisms of uptake (i.e., channel- or endocytosis-dependent) have been described in experiments utilizing different delivery methods, it is thought that the uptake mechanism depends not only on the method of delivery, but also the tissues examined. For example, in the case of dsRNA ingestion, SID-2 channels, which are expressed in the gut epithelium are involved in uptake of the molecules into the epithelial cells from the gut lumen. In a study of SID-2, it was found that the channel requires an acidic $\mathrm{pH}$ environment to effectively take up 
dsRNA, and that its luminal domain as well as extracellular, positively charged histidines, were important for its activity (McEwan et al., 2012). Surprisingly, when vesicle formation was inhibited, SID-2-dependent uptake of dsRNA was disrupted, suggesting that SID-2 is involved in the endocytic pathway. One of the functions of SID-1 is proposed to be transport of the dsRNA from the vesicles into the cytoplasm of the intestinal cell, where it can be processed by RAi machinery to induce silencing (McEwan et al., 2012).

It has been shown that when $C$. elegans takes up dsRNA from the gut lumen, silencing is not only observed in the intestinal cells, but also in other tissues in the nematode, and even in progeny, which occurs when dsRNA or its resultant siRNAs are imported into germ line cells (Jose and Hunter, 2007). While both SID-1 and SID-2 are known to be required for import of dsRNA into the cell, SID-2 has been ruled out for involvement in cell-to-cell transfer of the silencing trigger. SID-1, on the other hand, has been implicated in the spreading of the RNAi signal. In another experiment in C. elegans, GFP was expressed in the pharynx and in muscles along the body wall in wild-type and SID-deficient worms. The same worms were made to express dsGFP, transgenically, only in the pharynx, and were then fed on dsGFP. The wild-type animals showed silencing of GFP expression in both tissues, and so the signal was passed from the pharynx and gut to the body wall muscles. On the other hand, SID-deficient worms showed silencing of GFP only in the pharynx, demonstrating the signal had not been transferred from the pharynx or the gut, and that SID-1 channels are required for this to occur (Jose and Hunter, 2007; Winston et al., 2002). It has been shown, though, that transport of a silencing signal generated transgenically does not require SID-1, since animals that were SID-1-deficient in the pharynx, where GFP was silenced, passed the silencing signal to the SID-1-positive gut cells, effectively silencing GFP expression, but not to the SID-1-deficient body wall muscle cells, which would have been incapable of importing the signal (Jose et al., 2009). These findings, together with the apparent absence of SID genes in some animals, point to the potential variation in import and export mechanisms and the components in these pathways in different organisms, as well as the dependence on experimental approaches taken.

The research described above demonstrates that the two main mechanisms of dsRNA uptake do not function independently of one another, but work in conjunction in the context of RNAi. All of the described factors (i.e., uptake mechanism, initial target tissue), along with the organism in 
question and its corresponding mechanisms of and machinery for dsRNA import, export, and transfer, are interrelated, and together will determine the degree of effectiveness of RNAi.

\subsection{RNAi in insects: vector control considerations}

It is conceivable that administering dsRNA to an insect of significant public health or agricultural impact in order to interfere with its ability to exert its negative effects, would provide a means of insect control. While successful RNAi has been demonstrated in a number of insects from a wide range of orders, however, delivery of dsRNA at the population-scale in nature presents challenges. Most of the literature on the subject of RNAi and insect control describes its potential against plant-feeding insects that are agricultural pests; an application that is more feasible because plants can be made to express a construct which could potentially deter damage through its effect on the insect's system. While high knockdown efficiency has been demonstrated with oral delivery of dsRNA, one cannot control how much dsRNA will be ingested in nature from a plant, and therefore cannot predict the effectiveness of RNAi (Katoch et al., 2013). Moreover, the presence of enzymes capable of degrading dsRNAs in the gut, as well as the variable $\mathrm{pH}$ conditions in its different regions would likewise diminish the impact of RNAi, possibly reducing signal persistence. Another possible issue with RNAi as a mechanism of insect control is that of off-target effects, which can occur if the siRNAs produced following dsRNA processing in the cell share sequence identity with a non-target gene (Katoch et al., 2013). Finally, will the RNAi selectively impact pest species and not disrupt important functions in all insects indiscriminately, and if so, will all life stages be affected (Katoch et al., 2013)?

These questions and considerations are all transferrable to the discussion of RNAi application for controlling disease vectors, however, delivery of dsRNA poses a greater problem, and unsurprisingly, the research in this area is scarce. Nevertheless, some work has been done in this area proposing novel approaches. In $R$. prolixus, the gut microbiota was altered, through feeding on Escherichia coli expressing dsRNA against heme-binding protein (HBP) and catalase (CAT), to induce systemic RNAi (Taracena et al., 2015). A natural symbiont, Rhodococcus rhodnii, was also made to express dsRNA against the HBP construct, and orally administered to R. prolixus. Certain concentrations of $E$. coli expressing dsHBP and dsCAT elicited a strong reduction in corresponding gene expression ( $>90 \%)$ in the fat body and in the ovaries and posterior midgut, in both juveniles and adults. The molting cycle and oviposition were disrupted with delivery of both dsRNA signals (Taracena et al., 2015). With respect to dsRNA-expressing $R$. rhodnii, an 
$87 \%$ reduction in HBP expression was observed in the midgut and this was reflected in a reduction in oviposition. It was speculated that introduction of dsRNA in bacteria to the insects gut microbiota could be used to arrest vector function and development, and that this approach would circumvent the issue of non-specificity, thereby minimizing ecological impact (Taracena et al., 2015).

In conclusion, while efficient and prolonged RNAi has been demonstrated in different insects, including targeting different constructs and various means of delivery, the direct use of the procedure as a tool to control disease vector populations has yet to be tested in the field. The work would encounter issues related to effective delivery of dsRNA, which requires further testing. For example, determining if bacteria expressing dsRNA or its downstream products can survive on surfaces or skin, without adverse effects on the host, and can therefore be used on skin as an insecticide or repellent. For now, it appears that the greatest benefit that can come from RNAi experiments in the context of vector control is through identification of gene function and subsequent species-specific targets for control measures. 


\section{Objectives}

The aims of this work were as follows: (1) to silence expression of Rhopr-CRF/DH and RhoprCRF/DH-R2B and (2) validate silencing at the protein level; and (3) to examine feeding and reproductive behaviours in insects lacking Rhopr-CRF/DH and/or Rhopr-CRF/DH-R2B, with the overarching goal of investigating the importance of the CRF signalling-pathway in these vital processes in R. prolixus. 


\section{Materials and Methods}

\subsection{Animals}

Fifth instar $R$. prolixus, taken from a colony maintained at $25^{\circ} \mathrm{C}$ and high humidity $(50 \%)$, were used for this experiment. In feeding assays, insects were fed on defibrinated rabbit blood (Cedarlane, Burlington, CA).

\subsection{Double-stranded RNA (dsRNA) synthesis}

Using RNA extracted from unfed $5^{\text {th }}$ instar CNS tissues with the Total RNA Minipreps Superkit (Bio Basic Canada Inc., Markham, ON, Canada), cDNA was synthesized with the Applied Biosystems High Capacity cDNA Reverse Transcription Kit (Thermo Fisher Scientific Baltics UAB, Vilnius, Lithuania). PCR amplification with regular and T7 primers (Sigma-Aldrich Canada Co., Oakville, ON, Canada, Table 1) was performed and products were purified using the High Pure PCR Product Purification Kit (Roche, Pleasanton, CA, USA). Double stranded RNA targeting the peptide and receptors transcripts, dsCRF and dsCRFR, respectively, were synthesized with the T7 RiboMAX Express Large Scale RNA Production System (Promega, Madison, WI, USA). Control dsRNA, targeting the ampicillin resistance gene (ARG) was prepared following the same procedure, except PCR amplified the ARG gene from the pGEM-T Easy Vector Systems (Promega, Madison, WI, USA). dsRNA was diluted to a concentration of 1 $\mu \mathrm{g} / \mu \mathrm{L}$ in ultrapure distilled $\mathrm{H}_{2} \mathrm{O}$. 
Table 1. Primers used for synthesis of dsRNA. T7 promoter sequence is bolded.

\begin{tabular}{ll}
\hline Target & Sequence (5'-3') \\
\hline Rhopr-CRF/DH & Fw: GCGAACGTGTCTCTCAAC \\
& Rv: GTCAGCCTGTTTGTATGTCG \\
\hline T7-Rhopr-CRF/DH & Fw: TAATACGACTCACTATAGGGGCGAACGTGTCTCTCAAC \\
& Rv: TAATACGACTCACTATAGGGGTCAGCCTGTTTGTATGTCG \\
\hline Rhopr-CRF/DH-R2B & Fw: CTAGGGGAGGAAGAAATGAG \\
\hline T7-Rhopr-CRF/DH-R2B & Fw: TAATACGACTCACTATAGGGCTAGGGGAGGAAGAAATGAG \\
& Rv: TAATACGACTCACTATAGGGGCCCTTCTACAAACATCCA \\
\hline ARG & Fw: AATAGTTTGCGCAACGTTG \\
(Lee et al., 2013a) & Rv: ATGAGTATTCAACATTTCCGTGTC \\
\hline T7-ARG & Fw: TAATACGACTCACTATAGGGAGAAATAGTTTGCGCAACGTTG \\
(Lee et al., 2013a) & Rv: TAATACGACTCACTATAGGGAGAATGAGTATTCAACATTTCCGTGTC \\
\hline
\end{tabular}

\subsection{Injections}

Insects were immobilized on ice for $5 \mathrm{~min}$ and then injected with $1 \mu \mathrm{L}$ of $1 \mu \mathrm{g} \mathrm{dsCRF}$, dsCRFR, dsARG, or $1 \mu \mathrm{L}$ ultrapure distilled $\mathrm{H}_{2} \mathrm{O}$. Their CNS tissues were dissected in Dulbecco's PBS

(Sigma-Aldrich Inc., St. Louis, MO, USA) at days 1, 3, 5, and 7 post injection and stored in RNA Save (Biological Industries, Beit Haemek, Israel) until processing. RNA extraction and cDNA synthesis was performed using the same kits described in the previous section. 


\section{4 qPCR to determine knockdown efficiency}

$5 \mathrm{ng}$ of CNS cDNA was used in a reaction with SsoAdvanced Universal SYBR Green Supermix (Bio-Rad Laboratories Ltd., Mississauga, ON, Canada), to amplify Rhopr-CRF/DH transcript and reference gene transcripts (beta-actin, ribosomal protein 49, and tubulin*). The sequences of the primers used are listed in Table 2 (Sigma-Aldrich Canada Co., Oakville, ON, Canada). All primer efficiencies were $>90 \%$. The reactions were completed on the Mx4000 Quantitative PCR System (Stratagene, Mississauga, ON, Canada). Rhopr-CRF/DH transcript expression levels at various days post dsRNA injection were determined using the delta-delta $\mathrm{Ct}$ method, calculating expression at each day in dsCRF- and dsCRFR-injected insects relative to those injected with dsARG. Three biological replicates and no template controls were performed for each group (dsCRF, dsCRF, dsARG, and $\mathrm{H}_{2} \mathrm{O}$ ), day, and reference gene, and three technical replicates of the procedure were carried out.

*Tubulin was excluded as a reference gene from Rhopr-CRF/DH-R2B analysis.

Table 2. Primers for qPCR.

\begin{tabular}{ll} 
Target & Sequence $\left(5^{\prime}-3^{\prime}\right)$ \\
\hline Rhopr-CRF/DH & Fw: CAACCACCATTACAGGAATC \\
& Rv: GTCAGCCTGTTTGTATGTCG \\
\hline Rhopr-CRF/DH-R2B & Fw: GTGGATTCTTAGTTCTATTCAGGTG \\
\hline Rv: GCCCTTCTACAAACATCCA \\
\hline Fw: TCATCTCACCAATCTAACCCAC \\
Rv: CATTCCTACCATTACACCCTGAT \\
\hline Fhopr-rp49 & Fv: AGGAGAAATTGGCGCAAG \\
& Rv: GAAACCAGTAGGAAGCATGTGT \\
\hline
\end{tabular}




\subsection{Immunohistochemistry}

Procedure followed as described in Chapter 2, section 2.2. After CNS tissues were dissected in physiological saline, they were fixed in $2 \%$ paraformaldehyde for $1 \mathrm{~h}$ at room temperature, following which they were washed repeatedly in phosphate-buffered saline (PBS). Once the fix was washed off, tissues were permeabilized by incubating in 4\% Triton for $1 \mathrm{~h}$, then again washed thoroughly with PBS, and placed in primary antiserum solution containing polyclonal $L$. migratoria CRF-like (Locmi-DH) antisera at a concentration of 1:1000 in 0.4\% Triton and NGS for $48 \mathrm{~h}$. After incubation in primary antiserum solution, tissues were washed and placed in a secondary antibody solution containing Cy3-labelled sheep anti-rabbit immunoglobulin at a concentration of 1:600 in PBS and NGS overnight. The following day, after tissues were washed, they were incubated in increasing concentrations of glycerol (i.e., 20\%, 40\%, 60\%, 80\%, and 100\%) for 15 minutes and mounted on glass slides in 100\% glycerol. Confocal images were analyzed using ImageJ Software, acquiring mean grayscale values for the regions containing the NSCs.

\subsection{Feeding assay}

As described in Chapter 2, section 2.3. Fifth instars were injected with $1 \mu \mathrm{L}$ of $1 \mu \mathrm{g} / \mu \mathrm{L}$ dsRNA (dsCRF or dsARG) or $1 \mu \mathrm{L}$ ultrapure distilled $\mathrm{H}_{2} \mathrm{O}$. Five days later, when insects would have repressed expression by of the Rhopr-CRF/DH transcript by over $90 \%$, they were weighed, then placed to completion on defibrinated rabbit blood for $20 \mathrm{~min}$, following which they were immediately weighed to determine size of blood meal taken. They were then weighed again at hourly intervals for 5 hours to determine rate of weight loss, an indicator of rate of diuresis. In a separate experiment, insects were injected with $1 \mu \mathrm{L}$ of antiserum against Locmi-DH (final bleed, concentration unknown) or $1 \mu \mathrm{L}$ physiological saline. The same weighing and feeding procedure as describe above was followed.

\subsection{Statistical analyses}

qPCR data was analyzed with a two-way ANOVA followed by Bonferroni's test to compare mean transcript expression of Rhopr-CRF/DH and Rhopr-CRF/DH-R2B in insects injected with dsCRF and dsCRFR, respectively, relative to dsARG-injected controls. Immunohistochemical data was analyzed with the Student's $t$-test to determine the difference in mean grayscale values 
in the brain and MTGM between dsCRF and dsARG groups. Feeding data was likewise analyzed with the Student's $t$-test to compare pre- and post-feed body weights between insects injected with dsCRF and those injected with dsARG. Differences in diuresis rates between groups were examined by plotting weights of insects hourly post-feeding, fitting the data with regression lines, and running an F-test to determine whether the slopes differed significantly between treatment and control groups.

\section{Results}

\subsection{Rhopr-CRF/DH transcript knockdown efficiency and effects}

Expression of the Rhopr-CRF/DH transcript decreased in the CNS 1, 3, 5, and 7 days post injection of dsCRF by $82 \pm 2 \%, 73 \pm 1 \%, 91 \pm 1 \%$, and $95 \pm 2 \%(\mathrm{p} \leq 0.0001)$, respectively, relative to dsARG-injected controls (designated 100\%) (Fig. 1). Immunohistochemistry, however, showed that the knockdown of the transcript did not alter neuropeptide level. The intensity of Rhopr-CRF/DH-like immunostaining in the neurosecretory cells (NSCs) in the brain and mesothoracic ganglionic mass (MTGM), and their corresponding sites of release on the corpus cardiacum (CC) and abdominal nerves, respectively, did not differ between dsCRF- and dsARG-injected insects (mean grayscale value \pm SEM: $206.1 \pm 7$ vs. $213.8 \pm 8$, p $>0.05$ intensity of medial NSCs in the brain in dsARG- and dsCRF-injected insects, respectively; $184.9 \pm 5.7$ vs. $181.5 \pm 6.2, \mathrm{p}>0.05$ intensity of lateral NSCs in the MTGM in dsARG- and dsCRF-injected insects, respectively) (Fig. 2). Consistent with the immunohistochemical findings, there was no difference in size of blood meal taken by treatment and control groups 5 days after injection of dsRNA (mean post-feed weights \pm SEM: $261.6 \pm 7.5 \mathrm{mg}$ vs. $268.3 \pm 7.8 \mathrm{mg}, \mathrm{p}>0.05$ for dsARGand dsCRF-injected insects, respectively), nor did they differ in rates of diuresis (slope $\pm \mathrm{SEM}$ : $12.3 \pm 1.3$ vs. $-11.4 \pm 1.3, \mathrm{p}>0.05$ for dsARG- and dsCRF-injected insects, respectively) (Fig. 3A and B). Rhopr-CRF/DH transcript knockdown was determined to be $88 \pm 1 \%(\mathrm{p} \leq 0.0001)$.

An alternate method was taken to disrupt Rhopr-CRF/DH's normal function, by injecting antiserum against Locmi-DH 2-3 hours prior to feeding, and no difference in meal size or rate of diuresis between treatment and control groups was observed (mean post-feed weights $\pm \mathrm{SEM}$ : $252.6 \pm 15.5 \mathrm{mg}$ vs. $255.8 \pm 12.7 \mathrm{mg}$ ( $\mathrm{p}>0.05)$ of anti-Locmi-DH- and saline-injected insects, respectively) (Fig. 4 A and $\mathrm{B}$ ). 
Figure 1. Results of qPCR analysis showing the relative expression of the Rhopr-CRF/DH transcript in the CNS of $5^{\text {th }}$ instar $R$. prolixus injected with dsCRF or dsARG-injected controls at different time points post-injection of dsRNA.

There is a significant reduction in expression of Rhopr-CRF/DH in insects injected with dsRNA targeting the transcript, relative to controls ( $* * * *, p \leq 0.0001)$, with the most pronounced silencing observed 7 days after dsRNA injection. Error bars represent standard error of the mean across reference genes. 


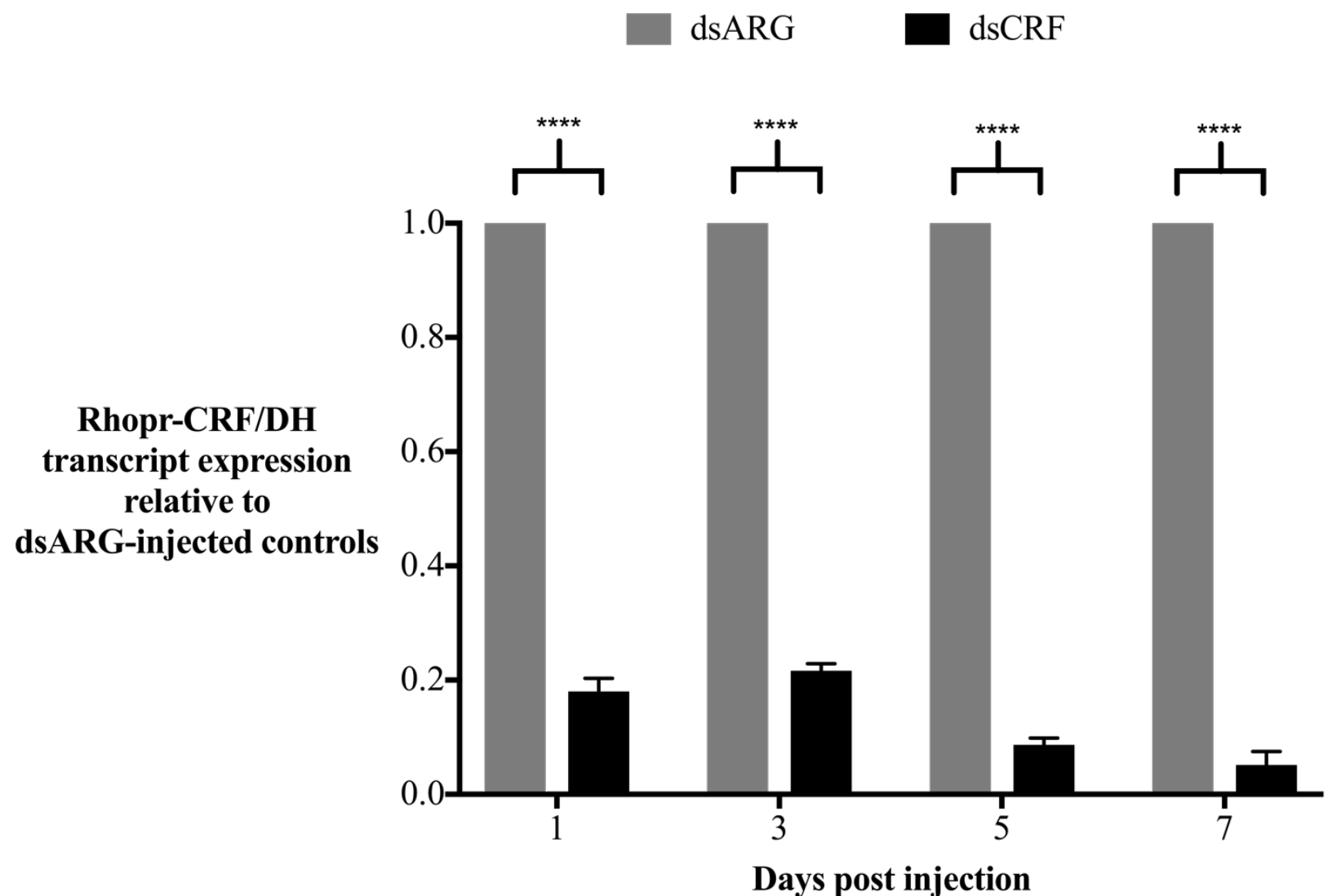


Figure 2. Results of immunohistochemistry showing Rhopr-CRF/DH-like immunostaining in the CNS of $5^{\text {th }}$ instar $R$. prolixus injected with dsCRF or dsARG 5 days prior.

Mean grayscale values, in arbitrary units, a measure of fluorescence intensity, did not differ between treatment and control groups $(p>0.05)$ in the medial neurosecretory cells (NSCs) of the brain (circled) and the lateral NSCs of the mesothoracic ganglionic mass (MTGM), and in their respective neurohaemal sites on the corpus cardiacum (CC) and abdominal nerves; this is confirmed visually in the confocal images. Error bars represent standard error of the mean; Scale bars $=100 \mu \mathrm{M}$. 
$\square \operatorname{dsCRF}(\mathrm{n}=5) \quad \operatorname{dsARG}(\mathrm{n}=5)$
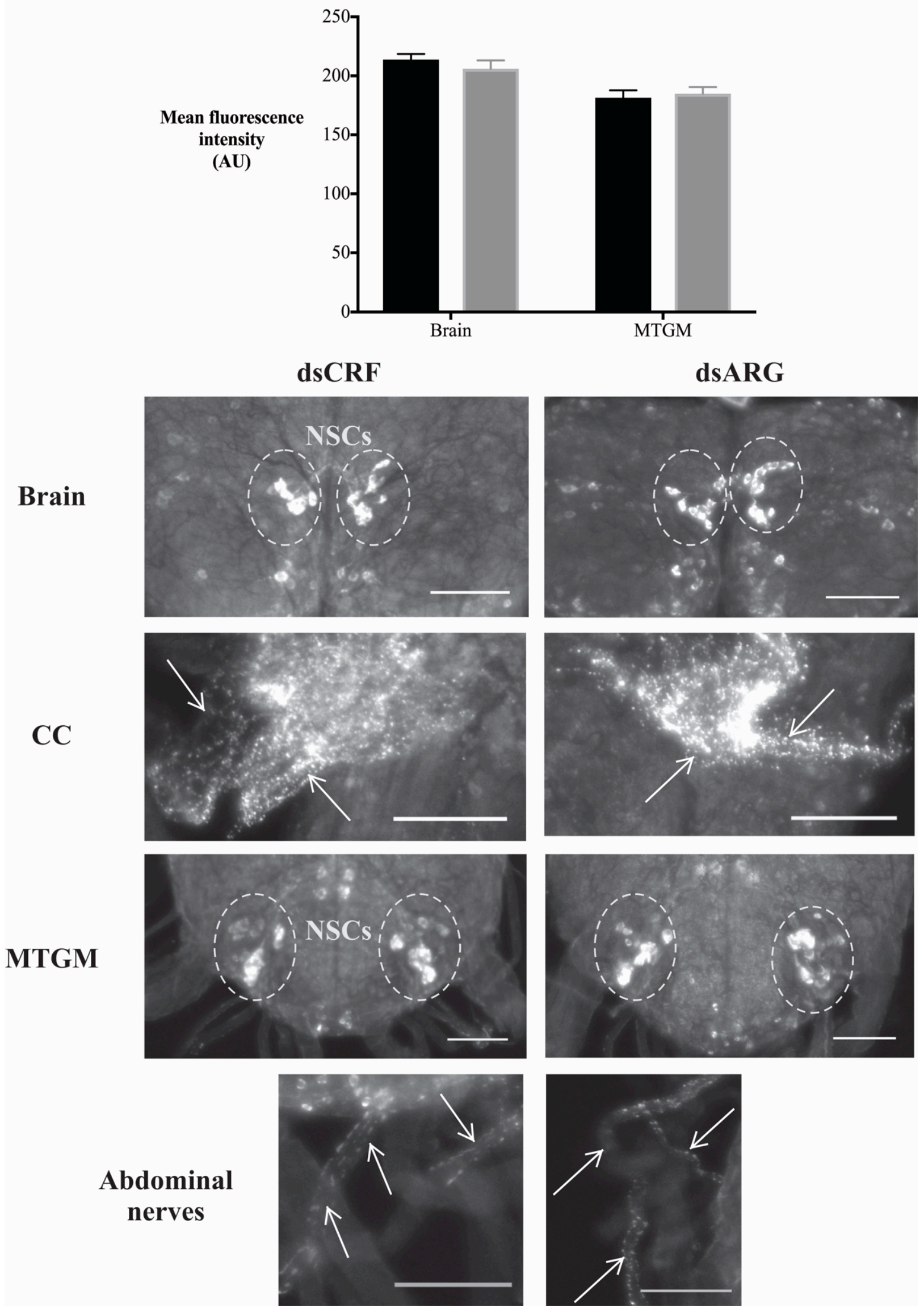


\section{Figure 3. Feeding assay following dsRNA injection.}

(A) Feeding assay performed on $5^{\text {th }}$ instar R. prolixus injected with dsCRF or dsARG 5 days prior. There was no difference in size of meal taken by treatment and control groups $(\mathrm{p}>0.05)$; both groups took a typical-sized meal, growing in size to 10 times their initial body weight. (B) Post-feed weights of dsCRF- and dsARG-injected insects were plotted with regression lines (i.e., linear dsCRF/dsARG), capturing their respective rates of weigh loss and thereby rates of diuresis, which did not differ significantly between the two groups $(\mathrm{p}>0.05)$. Error bars represent standard error of the mean. 
A

$\operatorname{dsARG}(\mathrm{n}=23) \square \operatorname{dsCRF}(\mathrm{n}=26)$

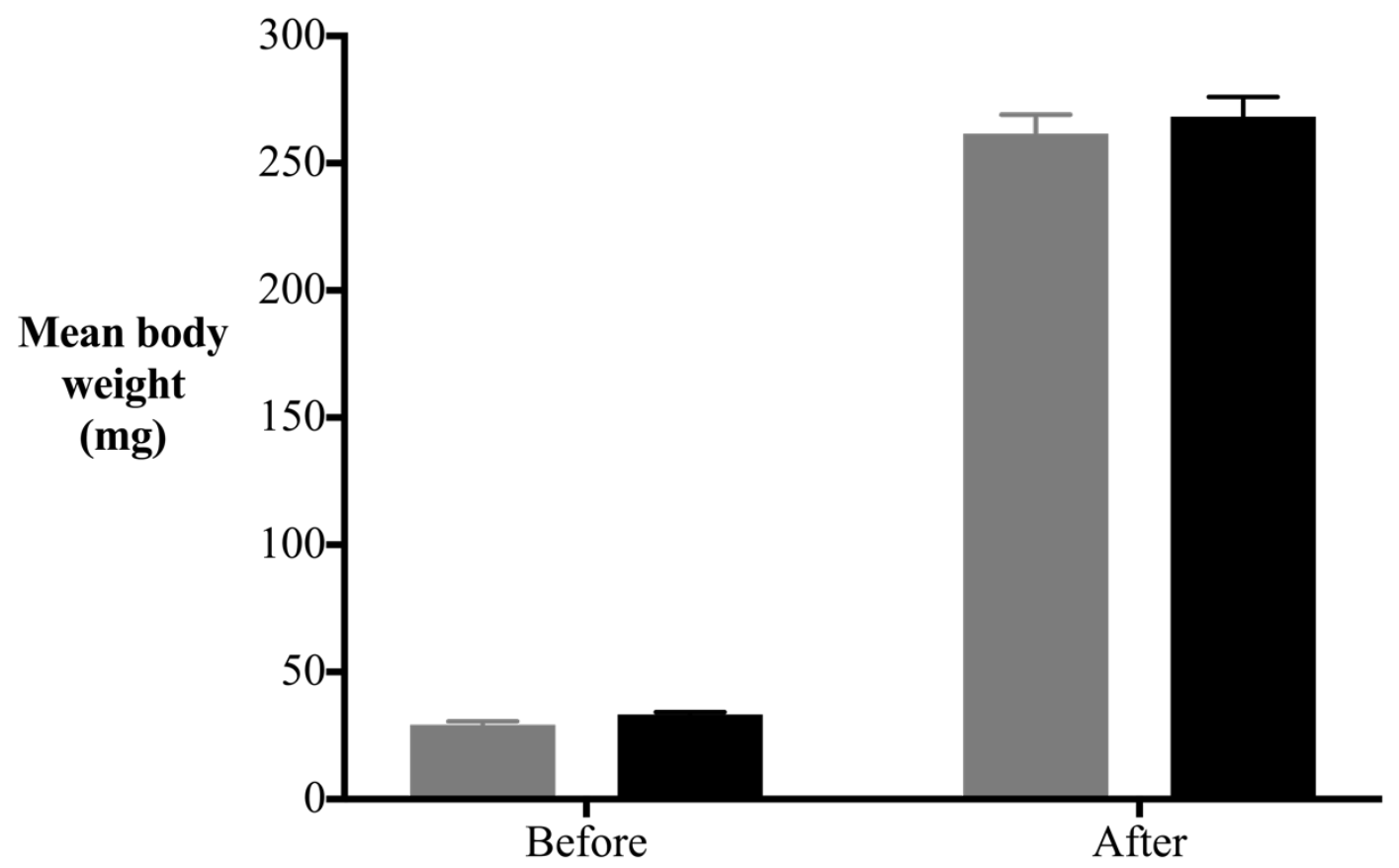

B

dsARG $(\mathrm{n}=23) \quad \mathrm{dsCRF}(\mathrm{n}=26)$

--. Linear dsARG --. Linear dsCRF

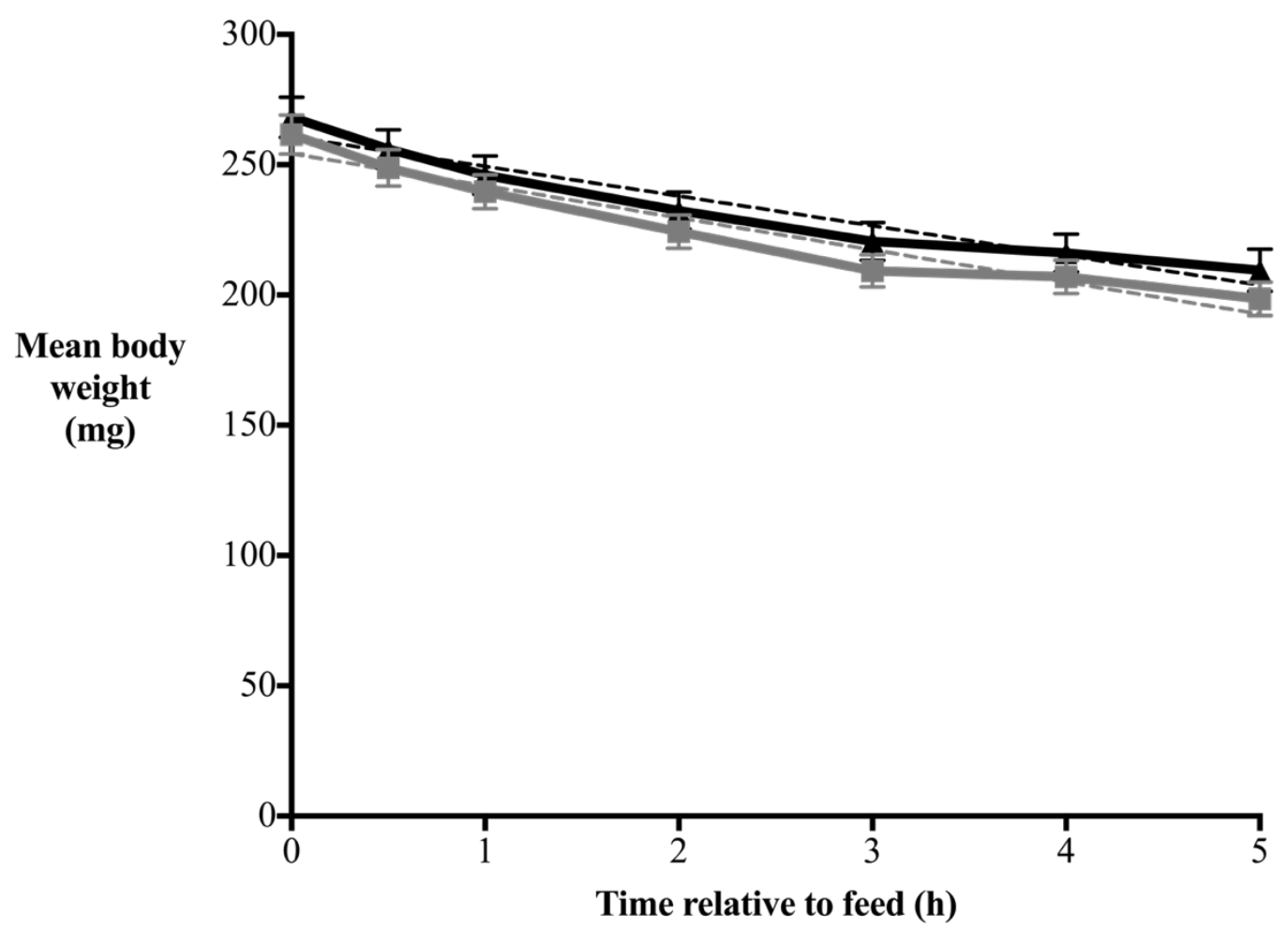




\section{Figure 4. Feeding assay following injection of antiserum.}

(A) Feeding assay performed on $5^{\text {th }}$ instar R. prolixus injected with anti-Locmi-DH or saline 2-3 hours prior to feeding. There was no difference in size of meal taken by treatment and control groups ( $>0.05$ ); both groups took a typical-sized meal, growing in size to 10 times their initial body weight. (B) Post-feed weights of anti-Locmi-DH- and saline-injected insects were plotted with regression lines (i.e., linear anti-Locmi-DH/saline), capturing their respective rates of weigh loss and thereby rates of diuresis, which did not differ significantly between the two groups $(p>0.05)$. Error bars represent standard error of the mean. 
A

$$
\text { Saline (n=14) } \square \text { Anti-Locmi-DH (n=23) }
$$

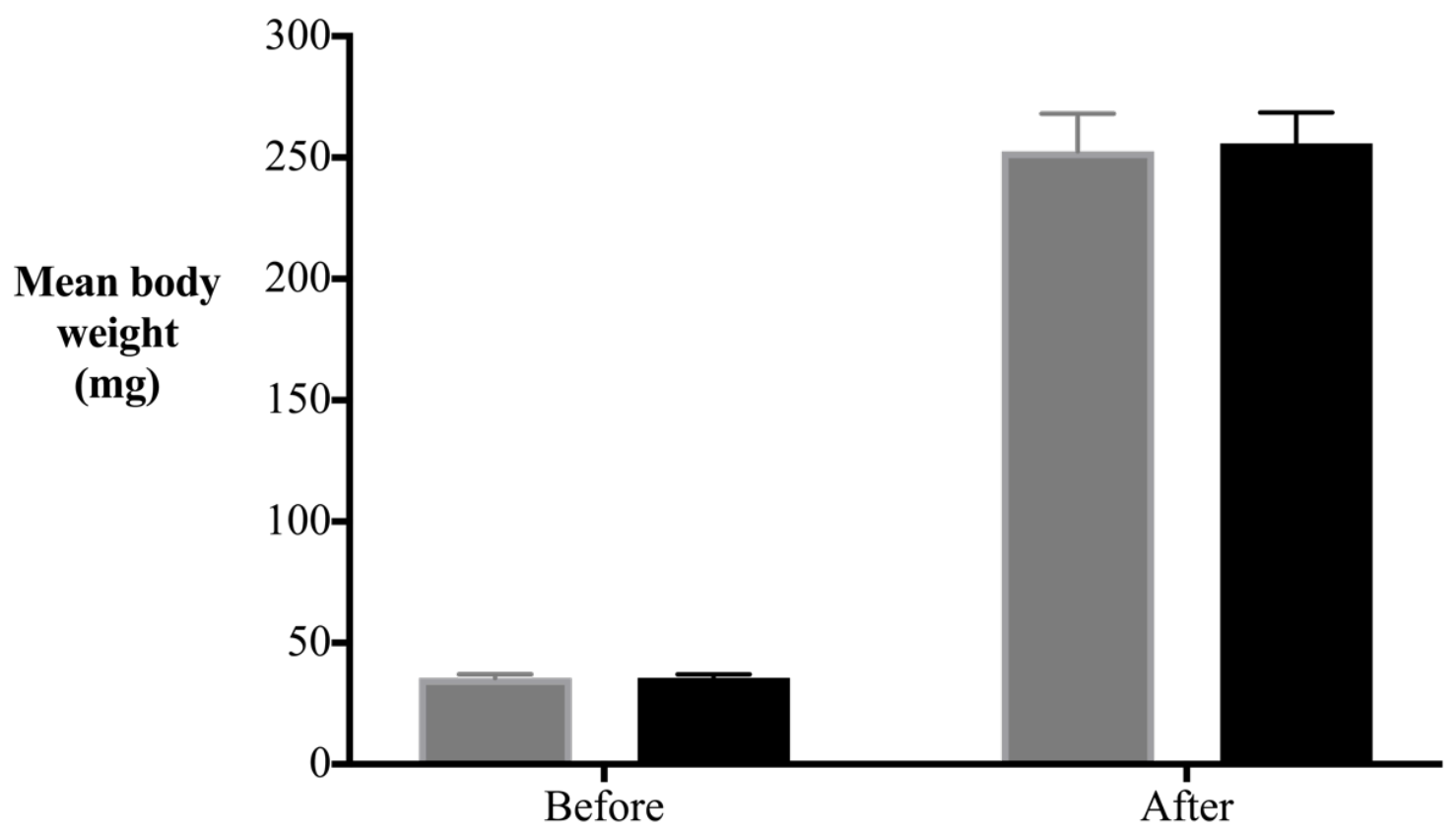

Time relative to feed

B

$\rightarrow$ Saline $(\mathrm{n}=14) \rightarrow$ Anti-Locmi-DH $(\mathrm{n}=23)$

--. Linear Saline $\quad$--. Linear Anti-Locmi-DH

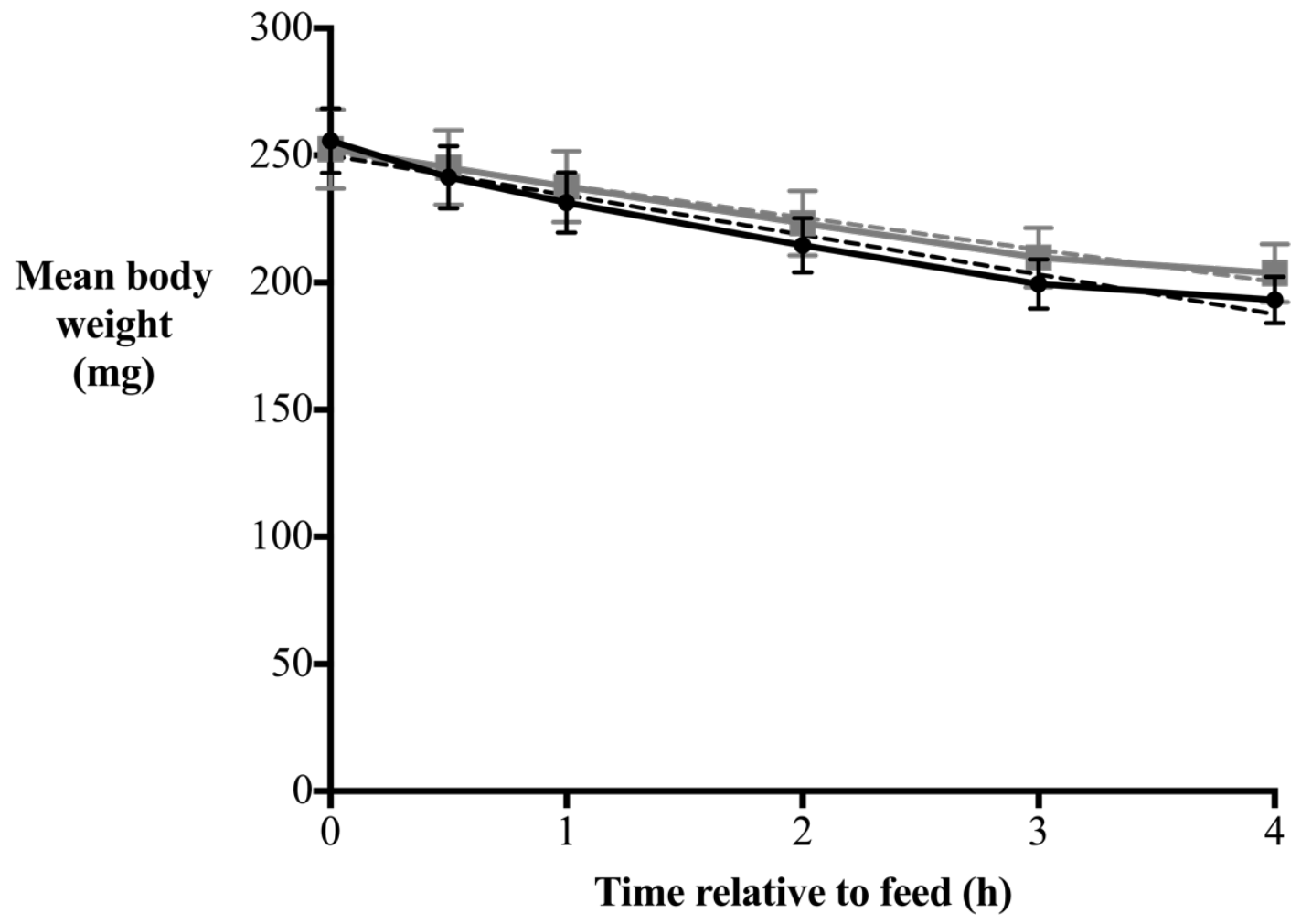




\subsection{Rhopr-CRF/DH-R2B transcript knockdown}

Expression of the Rhopr-CRF/DH-R2B transcript decreased in the CNS 2, 3, and 5 days post injection of dsCRFR by $18 \pm 3 \%\left(^{*}, \mathrm{p} \leq 0.05\right), 39 \pm 6 \%$, and $33 \pm 3 \%(* * *, p \leq 0.001)$, respectively, relative to dsARG-injected controls (Fig. 5). 
Figure 5. Results of qPCR analysis, showing the relative expression of the Rhopr-CRF/DHR2B transcript in the CNS of $5^{\text {th }}$ instar $R$. prolixus injected with dsCRFR or dsARGinjected controls at different time points post-injection of dsRNA.

There is a significant reduction in expression of Rhopr-CRF/DH-R2B in insects injected with dsRNA targeting the receptor transcript, relative to controls $(*, \mathrm{p} \leq 0.05$ on day 2 and $* * *$, $\mathrm{p} \leq 0.0001$ on days 3 and 5), with the most pronounced silencing observed 3 days after dsRNA injection. Error bars represent standard error of the mean across reference genes. 


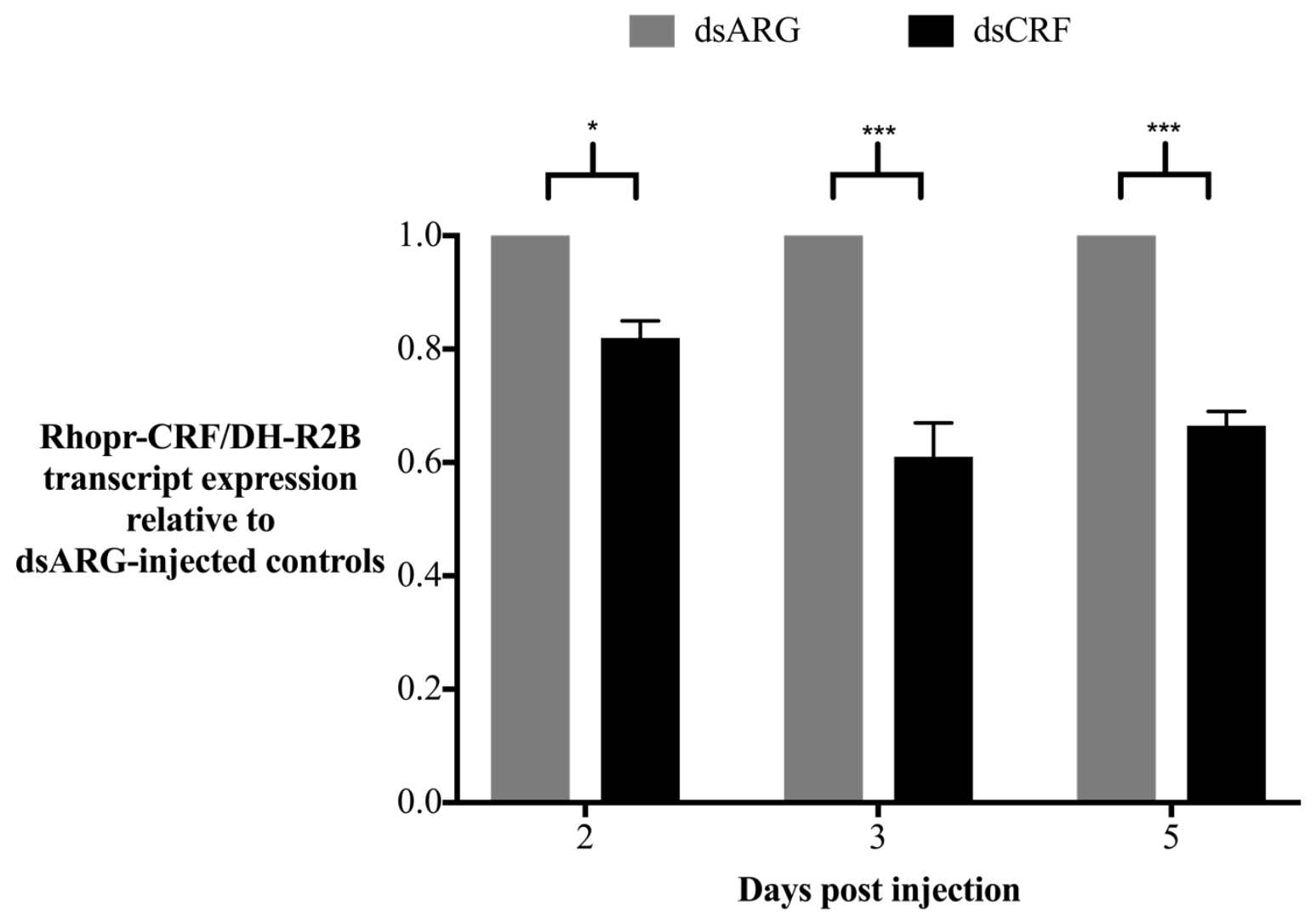




\section{Discussion}

The goal was to use molecular techniques to silence Rhopr-CRF/DH and/or its receptor RhoprCRF/DH-R2B and observe the effect in behavioural and physiological assays. Knockdown of the Rhopr-CRF/DH transcript, while highly efficient, did not translate into decreasing content of the neuropeptide in the NSCs which restricted analysis of its function in R. prolixus. While an over $90 \%$ decrease in expression of Rhopr-CRF/DH transcript was observed 5 days after dsRNA injection, and this knockdown persisted to day 7, there was no difference in Rhopr-CRF/DH-like immunostaining in the CNS in the NSCs of the MTGM, which have been implicated in the process of feeding, nor in the medial NSCs in the brain. Consistent with the absence of neuropeptide reduction, there was no difference in size of meal taken by insects injected with dsCRF and those injected with dsARG. With respect to the receptor Rhopr-CRF/DH-R2B, knockdown efficiency was not as high as it was for the peptide transcript, reaching its highest of $39 \% 3$ days after injection, and without an antibody for the receptor protein, analysis of correspondence between knockdown of transcript and reduction of receptor was not performed. Based on what is known about the relationship between transcript and protein levels and their relative stabilities, one can propose an explanation for the observed results and possible solutions for the issues, which may allow progression of the planned work.

The aforementioned correlation between transcript and peptide/protein levels is highly variable, dependent on a number of factors such as the molecule in question, the regulation of its production and degradation and consequent half-life, and the organism and tissue being examined. In general this relationship shows a $40 \%$ correlation, that is, transcript level explains about $40 \%$ of the variation in protein abundance, the other $60 \%$ determined by posttranscriptional regulation, translational regulation, and degradation of proteins (Vogel and Marcotte, 2012). The finding, in the present work, that knockdown of the Rhopr-CRF/DH transcript is not reflected in protein levels, could be attributed to the long half-life of this protein. The stores of the neurohormone in the NSCs in the brain, particularly the lateral NSCs of the MTGM, are depleted after feeding, since that is when Rhopr-CRF/DH is released to exert its effects, as a diuretic hormone as well as a signal for the termination of feeding. Some bloodfeeding insects, among them bed bugs and triatomine bugs such as $R$. prolixus in nature can survive long periods without feeding (Benoit et al., 2016). R. prolixus in the laboratory feed 
infrequently, every 4+ weeks. A single feed, where a nymph takes a blood meal up to 10 times its initial body weight, is sufficient for the insect to go through the moult cycle into the next life stage. As such, it is plausible that expression of Rhopr-CRF/DH is likewise infrequent, not constantly synthesized, but increasing only when the Rhopr-CRF/DH stores in the CNS are depleted following feeding, to restock and store until the next feed, which is consistent with the observed increase in Rhopr-CRF/DH transcript expression in the MTGM immediately following feeding (Mollayeva et al., 2017). Therefore, when expression was downregulated via RNAi, because Rhopr-CRF/DH was already in the NSCs, made following the previous feed, it was still available for release following feeding to carry out its usual function, stimulating absorption in the midgut, secretion by the Malpighian tubules, and termination of feeding.

Consistent with this thought, the ideal RNAi target has been described as a gene producing mRNA with a high turnover rate and coding for a short-lived protein, as protein products with a long half-life would impede investigation of gene function by phenotypic analysis (Scott et al., 2013). In a study of a nicotinic acetylcholine receptor (nAChR) in D. melanogaster and $T$. castaneum, specifically the $\alpha 6$ subunit, which is a target of insecticide spinosad, RNAi was utilized to downregulate expression and spinosad sensitivity was subsequently analyzed. While a knockdown of $60-75 \%$ and $50 \%$ for the $\mathrm{nAChR}$ transcript was achieved for D. melanogaster and T. castaneum, respectively, no change in spinosad sensitivity was observed in the animals. The rate of turnover of $\mathrm{nAChR}$, which can be stable for over 2 weeks, was proposed as a possible reason for the failure of RNAi to induce a phenotypic effect (Rinkevich and Scott, 2013).

A possible approach to resolving the issue encountered in the present work is to bypass the disconnect between mRNA and protein that exists in the CNS with respect to Rhopr-CRF/DH, due to the neurohormone's presumably long half-life in the NSCs. If dsRNA is injected into the heamolymph prior to $R$. prolixus feeding, once feeding has terminated and Rhopr-CRF/DH has been released from the NSCs, the increased expression of mRNA for restocking of the cells could be disrupted due to presence of dsRNA targeting it for degradation. This approach will likely involve its own barriers. Firstly, as previously mentioned, $R$. prolixus nymphs take a huge blood meal, and haemolymph volume increases as a result of absorption by the midgut and reabsorption by the Malpighian tubules. As such, successful injection of Rhopr-CRF/DH may be challenging, as piercing of the cuticle would result in haemolymph being drawn out and the contents of the injection may not be taken up completely or at all. Secondly, in order to perform 
the behavioural assays to investigate the role of Rhopr-CRF/DH in feeding and reproductive processes, it is necessary to validate that knockdown of the peptide and the resultant suppression of protein synthesis persists long enough (e.g., immunohistochemistry, western blot, etc.). In the case of the feeding assay, for example, the effects of the initial feed, following which Rhopr$\mathrm{CRF} / \mathrm{DH}$ stores would be depleted and subsequent RNAi to prevent restocking, would need to persist 4+ weeks until the subsequent feed, when the insects would be starved and able to take a blood meal once again. Finally, if repeated injection of dsRNA was to be performed to maintain knockdown, the risk of mechanical damage and death rises, as does the possibility of measurement inaccuracies due to disruption of the systems' homeostasis. This is particularly relevant to measurement of Rhopr-CRF/DH, which may, as it does in mammals, act as a stress response hormone in insects. Another possible route would involve looking at the effects of different dilutions of the Locmi-DH antiserum when injected prior to feeding. The antiserum would be presumed to be in circulation in the haemolymph at the time of feeding, and when endogenous Rhopr-CRF/DH would be released, it would be bound by the antibody and prevented from exerting its effects.

While the purpose of the RNAi in this work was to elucidate the role of Rhopr-CRF/DH in feeding and reproduction in $R$. prolixus, with the ultimate goal of identifying a significant target for vector control efforts (i.e., alters major processes such as feeding, diuresis, reproduction), the RNAi method itself has been described as a potential tool for insect control. There are a number of barriers, however, keeping it from application, namely in disease vectors, where method of dsRNA delivery would be complicated in a natural environment. Nevertheless, using RNAi indirectly to inform more effective means of insect control is promising, highlighting novel species-specific targets and thereby reducing the ecological impact through non-specific effects on non-target, and potentially ecologically significant, insect populations. 


\section{References}

Benoit, J.B., Adelman, Z.N., Reinhardt, K., Dolan, A., Poelchau, M., Jennings, et al., 2016. Unique features of a global human ectoparasite identified through sequencing of the bed bug genome. Nat. Commun. 7, 10165. doi:10.1038/ncomms 10165

Burand, J.P., Hunter, W.B., 2013. RNAi: Future in insect management. J. Invertebr. Pathol. doi:10.1016/j.jip.2012.07.012

Cappelle, K., De Oliveira, C.F.R., Van Eynde, B., Christiaens, O., Smagghe, G., 2016. The involvement of clathrin-mediated endocytosis and two Sid-1-like transmembrane proteins in double-stranded RNA uptake in the Colorado potato beetle midgut. Insect Mol. Biol. 25, 315323. doi:10.1111/imb.12222

Cenik, E.S., Zamore, P.D., 2011. Quick guide: Argonaute proteins. Curr. Biol. 21. R446-449. doi: 10.1016/j.cub.2011.05.020

Defferrari, M.S., Orchard, I., Lange, A.B., 2016. Identification of the first insulin-like peptide in the disease vector Rhodnius prolixus: Involvement in metabolic homeostasis of lipids and carbohydrates. Insect Biochem. Mol. Biol. 70, 148-159. doi:10.1016/j.ibmb.2015.12.009

Diederichs, S., Haber, D.A., 2007. Dual Role for Argonautes in MicroRNA Processing and Posttranscriptional Regulation of MicroRNA Expression. Cell 131, 1097-1108. doi:10.1016/j.cell.2007.10.032

Huvenne, H., Smagghe, G., 2010. Mechanisms of dsRNA uptake in insects and potential of RNAi for pest control: A review. J. Insect Physiol. doi:10.1016/j.jinsphys.2009.10.004

Ishizu, H., Siomi, H., Siomi, M.C., 2012. Biology of Piwi-interacting RNAs: New insights into biogenesis and function inside and outside of germlines. Genes Dev.

doi:10.1101/gad.203786.112

Jinek, M., Doudna, J.A., 2009. A three-dimensional view of the molecular machinery of RNA interference. Nature 457, 405-412. doi:10.1038/nature07755

Jose, A.M., Hunter, C.P., 2007. Transport of sequence-specific RNA interference information between cells. Annu Rev Genet 41, 305-330. doi:10.1146/annurev.genet.41.110306.130216

Jose, A.M., Smith, J.J., Hunter, C.P., 2009. Export of RNA silencing from C. elegans tissues does not require the RNA channel SID-1. Proc. Natl. Acad. Sci. U. S. A. 106, 2283-2288. doi:10.1073/pnas.0809760106

Katoch, R., Sethi, A., Thakur, N., Murdock, L.L., 2013. RNAi for insect control: Current perspective and future challenges. Appl. Biochem. Biotechnol. 171, 847-873. doi:10.1007/s12010-013-0399-4 
Lee, D., Orchard, I., Lange, A.B., 2013. Evidence for a conserved CCAP-signaling pathway controlling ecdysis in a hemimetabolous insect, Rhodnius prolixus. Front. Neurosci. doi:10.3389/fnins.2013.00207

Lee, D., Vanden Broeck, J., Lange, A.B., 2013a. Identification and Expression of the CCAP Receptor in the Chagas' Disease Vector, Rhodnius prolixus, and Its Involvement in Cardiac Control. PLoS One 8. doi:10.1371/journal.pone.0068897

Lewis, B.P., Burge, C.B., Bartel, D.P., 2005. Conserved seed pairing, often flanked by adenosines, indicates that thousands of human genes are microRNA targets. Cell 120, 15-20. doi:10.1016/j.cell.2004.12.035

McEwan, D.L., Weisman, A.S., Hunter, C.P., 2012. Uptake of Extracellular Double-Stranded RNA by SID-2. Mol. Cell 47, 746-754. doi:10.1016/j.molcel.2012.07.014

Mollayeva, S., Orchard, I., Lange, A.B., 2017. The involvement of Rhopr-CRF/DH in feeding and reproduction in the blood-gorging insect Rhodnius prolixus. Gen. Comp. Endocrinol. doi: 10.196/j.ygcen.2017.07.005 (in press)

Paim, R.M.M., Araujo, R.N., Lehane, M.J., Gontijo, N.F., Pereira, M.H., 2013. Long-term effects and parental RNAi in the blood feeder Rhodnius prolixus (Hemiptera; Reduviidae). Insect Biochem. Mol. Biol. 43, 1015-1020. doi:10.1016/j.ibmb.2013.08.008

Rinkevich, F.D., Scott, J.G., 2013. Limitations of RNAi of $\alpha 6$ nicotinic acetylcholine receptor subunits for assessing the in vivo sensitivity to spinosad. Insect Sci. 20, 101-108. doi:10.1111/j.1744-7917.2012.01523.x

Scott, J.G., Michel, K., Bartholomay, L.C., Siegfried, B.D., Hunter, W.B., Smagghe, G., Zhu, K.Y., Douglas, A.E., 2013. Towards the elements of successful insect RNAi. J. Insect Physiol. doi:10.1016/j.jinsphys.2013.08.014

Taracena, M.L., Oliveira, P.L., Almendares, O., Umaña, C., Lowenberger, C., Dotson, E.M., Paiva-Silva, G.O., Pennington, P.M., 2015. Genetically Modifying the Insect Gut Microbiota to Control Chagas Disease Vectors through Systemic RNAi. PLoS Negl. Trop. Dis. 9. doi:10.1371/journal.pntd.0003358

Vogel, C., Marcotte, E.M., 2012. Insights into the regulation of protein abundance from proteomic and transcriptomic analyses. Nat. Rev. Genet. 13, 227-32. doi:10.1038/nrg3185

Winston, W.M., Molodowitch, C., Hunter, C.P., 2002. Systemic RNAi in C. elegans requires the putative transmembrane protein SID-1. Science 295, 2456-9. doi:10.1126/science.1068836

Winston, W.M., Sutherlin, M., Wright, A.J., Feinberg, E.H., Hunter, C.P., 2007. Caenorhabditis elegans SID-2 is required for environmental RNA interference. Proc. Natl. Acad. Sci. U. S. A. 104, 10565-70. doi:10.1073/pnas.0611282104 


\section{Chapter 4 \\ General Discussion}

\section{Rhopr-CRF/DH and feeding}

In this work, the hypothesized involvement of Rhopr-CRF/DH in feeding was supported by data showing that when haemolymph titres of the neurohormone were elevated prior to feeding by injection, Rhodnius prolixus took in a significantly smaller blood meal, relative to saline-injected controls. No differences in rates of diuresis were observed between treatment and control groups, and treatment individuals did not appear to commence diuresis sooner. Moreover, when serotonin, a diuretic hormone that works with Rhopr-CRF/DH to stimulate anterior midgut absorption and Malpighian tubule section, did not alter feeding behaviour when injected prior to feeding. These findings suggest that Rhopr-CRF/DH's role in feeding is temporally and spatially (i.e., activating its receptor on different target tissues, or involved in a feedback loop (Chapter x; Mollayeva et al., 2017) distinct from its activity as a diuretic factor.

When dsRNA targeting the Rhopr-CRF/DH transcript was administered to $5^{\text {th }}$ instars by injection, no difference was observed in size of blood meal taken relative to dsARG-injected controls. Following immunohistochemical analysis, this result was unsurprising. Rhopr$\mathrm{CRF} / \mathrm{DH}-$ like immunostaining did not differ in the neurosecretory cells (NSCs) of the mesothoracic ganglionic mass (MTGM) between treatment and control groups, suggesting Rhopr-CRF/DH has a long half-life within the cell, as knockdown of the transcript was not reflected in levels of peptide. Thus, Rhopr-CRF/DH, synthesized and stored in the NSCs following the insects' previous feed, was available for release from the abdominal nerve neurohaemal sites of the MTGM during the tested feeding and was able to exert its normal effects with respect to feeding and diuresis.

Rhopr-CRF/DH's modulation of the feeding process in $R$. prolixus is in line with what has previously been observed in other insects, namely in Locusta migratoria (Audsley et al., 1997), Schistocerca gregaria (van Wielendaele et al., 2012), and Heliothis virescens (Gäde and Goldsworthy, 2003). The mechanism underlying this effect is not fully known, but one can speculate that, as is the case in mammals, CRF is a signalling molecule in insects with multiple downstream signalling pathways that can culminate in reduced feeding. For example, in mammals, leptin is a regulator of energy balance and a known satiety factor, and its release is 
stimulated by glucocorticoids, a downstream component of the CRF pathway (Heiman et al., 1997). Also in mammals, CRF peptides were found to decrease circulating levels of appetitestimulating ghrelin (Yakabi et al., 2011). In R. prolixus, DH derived from Zootermopsis nevadensis (Zoone-DH) was found to increase midgut contractions (Te Brugge et al., 2009). While this was originally described in the context of post-prandial diuresis, it is possible that contraction of the midgut is involved in the regulation of feeding, signalling termination by starting to process the blood meal, as Zoone DH was also found to induce ion and water movement across the anterior midgut (Te Brugge et al., 2009). In mammals, a link between CRF and gastric motility was likewise described, however, the neurohormone there inhibited gastric contractions and emptying (Stengel and Tache, 2014), suggesting an alternate mode of feeding inhibition. Another possible mode of action is through stretch receptors, which are located on the abdominal wall and sense gut distension (Simpson and Bernays, 1983). In locusts and blowflies, these receptors have been implicated in meal termination, signalling when the gut has been stretched to capacity and is full. In blowflies, stretch responsive neurones also innervate the oesophagus, and when these nerves were severed, the result was overeating. Input from stretch receptors has not only direct effects with respect to feeding inhibition, but also indirect effects through other signalling molecules. Activation of the stretch receptors results in neuroendocrine activation through release of hormones from the corpus cardiacum (CC) (Simpson and Bernays, 1983). Specifically, the release of DH is correlated with abdominal distension (Chiang and Davey, 1988; Simpson and Bernays, 1983). While this again has been described in the context of diuresis, based on the finding that Rhopr-CRF/DH inhibits feeding in R. prolixus, and that its release and activity during feeding is likely separate from its role as a diuretic hormone (since serotonin, another diuretic hormone, administered at the same time did not impact feeding) (Chapter X; Mollayeva et al., 2017), it is conceivable that activation of stretch receptors due to abdominal distension from a large blood meal and subsequent release of diuretic hormone is a signal terminating feeding. Finally, Locmi-DH has been shown to alter sensitivity to food in $L$. migratoria, by inducing closure of apical pores on the taste sensilla (Gäde and Goldsworthy, 2003). Injection of Locmi-DH antiserum blocked this effect. Prior to identification of this DH in locusts, $\mathrm{CC}$ extracts were shown to have the aforementioned affect on taste sensilla (Bernays and Mordue, 1973). Rhopr-CRF/DH is likewise found at neurohaemal sites in the CC and may thus be used as a similar mechanism to terminate feeding. 


\section{Rhopr-CRF/DH and reproduction}

Experiments investigating the effect of Rhopr-CRF/DH on reproductive capacity likewise supported the hypothesized multifaceted role of the neurohormone in R. prolixus. Mated adult females injected with Rhopr-CRF/DH both produced and laid significantly fewer eggs than did their saline-injected counterparts. Moreover, females injected with Rhopr-CRF/DH appeared to experience a plateau in their rate of egg laying sooner than did control females, suggesting Rhopr-CRF/DH diminishes reproductive capacity.

A possible mechanism underlying this attenuated oviposition in response to Rhopr-CRF/DH injection is inhibition of oviduct contractions, since a dose-dependent decrease in force of contractions was observed in the lateral oviducts with application of the neurohormone. In $R$. prolixus, ovulation and oviposition require the activity of a myotropic hormone found in NSCs in the brain, to increase contractions of the muscles surrounding the ovaries (Ampleford and Davey, 1989). Moreover, the size of the sphincter connecting the ovary and the lateral oviduct must also be regulated to aid in ovulation and oviposition (Ampleford and Davey, 1989). Thus, Rhopr$\mathrm{CR} / \mathrm{DH}$, by disrupting this contractile activity, could disrupt these processes.

While these specific results have not been described in other insects, the general reduction in reproductive capacity triggered by CRF is consistent with previous work. In S. gregaria, injection of Schgr-CRF/DH into sexually mature females resulted in reduced oocyte size and lower levels of circulating ecdysteroids (van Wielendaele et al., 2012). Injection of dsRNA targeting OMP, which is coded on the same gene as CRF, reversed these effects. Moreover, among the different tissue targets of Rhopr-CRF/DH in $R$. prolixus are the female reproductive tissues. The receptor, Rhopr-CRF/DH-R2B, is expressed in these tissues and this expression is significantly higher in the ovaries of adult females relative to immature ovaries in $5^{\text {th }}$ instars (Lee et al., 2016). These data implicate Rhopr-CRF/DH in the regulation of reproduction, as this process is a major distinction between juvenile and adult and would thus explain the change in expression. This also highlights a mechanism of action with respect to the observed decrease in oogenesis by females injected with Rhopr-CRF/DH, through the ovaries, in which increased expression of the receptor was observed. Oocytes grow in the ovary by accumulating material from the trophic core, and then, later vitellogenesis takes place, by which they acquire yolk 
protein from the haemolymph (Davey, 1981). Rhopr-CRF/DH, by binding to its receptors on the ovaries, could interfere with these processes, retard oogenesis, ovulation, and oviposition.

In vertebrates, the inhibitory effects of $\mathrm{CRF}$ with respect to reproduction have been more extensively described, as CRF is classified as a stress response hormone and reproductive capacity diminishes in response to stress, most notably through suppression of the hypothalamicpituitary-gonadal axis (Kinsey-Jones et al., 2010; Kovacs, 2013; Li et al., 2005). Intracerebral administration of CRF into female rats inhibited ovulation, and reduced LH levels (Rivier and Vale, 1984), which would impact follicular maturation and ovulation, which LH is known to regulate (Choi and Smitz, 2014). Rhopr-CRF/DH may likewise inhibit reproductive processes by suppressing the effects of juvenile hormone, which is the major regulator of reproduction in $R$. prolixus (Davey, 1993).

\section{Rhopr-CRF/DH and the stress response in insects}

Given Rhopr-CRF/DH's apparently multifaceted role modulating vital processes that can alter the homeostatic balance of $R$. prolixus, it is conceivable that this neurohormone, as it is in vertebrates, is a stress hormone in insects, signalling to the system to terminate stress-triggering activity, put off energy-consuming processes such as reproduction, and ultimately restore balance. This is supported by Rhopr-CRF's localization in NSCs in the brain, which project to and release the neurohormone from the neurohaemal sites of the CC, and NSCs in the MTGM, which project to the abdominal nerves and from where Rhopr-CRF/DH is likewise released into the haemolymph. Once in circulation, it can activate its receptor pathways on any number of tissues, exerting a variety of effects, among them the aforementioned effects on feeding and reproduction. The stress response in insects is not fully understood and has not been well described; however, a model has been proposed describing the stress response in the honey bee A. mellifera (Even et al., 2012). There, once a stressful stimulus has been detected by sensory organs, activation of NSCs in the brain and subsequent release of neurohormones from the CC will increase arousal and mobilize energy stores to trigger the "fight-or-flight" response. Among these hormones, CRF homolog DH-1 is suggested to be involved (Even et al., 2012). Two hormones in particular, octopamine and adipokinetic hormone $(\mathrm{AKH})$ are believed to be stress hormones in insects, the former the equivalent of norepinephrine which is synthesized in the LC, a downstream target of CRF in mammals (Sun et al., 2015). LC activity is involved in generation 
of anxiogenic and aversive behaviours, innervating a wide range of centers implicated in these behavioural responses to stress (Sun et al., 2015). In the locust, haemolymph octopamine levels were found to increase following exposure to a variety of stressors (i.e., mechanical, thermal, chemical) (Davenport and Evans, 1984). The second insect stress hormone, AKH, is a major regulator of energy mobilization and thus, development, reproduction, and the stress response (Galinkova et al., 2015). AKH-deficient D. melanogaster are overweight and hypoglycemic, while the presence of $\mathrm{AKH}$ interferes with oogenesis and vitellogenesis in L. migratoria and Gryllus bimaculatus. It is possible that octopamine and $\mathrm{AKH}$ are downstream components in the Rhopr-CRF/DH pathway in R. prolixus, or that they work in conjunction with each other to respond to stress and maintain homeostasis. Endogenously, Rhopr-CRF/DH may signal termination of feeding once the insect has taken a sufficient meal directly, through activation of stretch receptors to mitigate further mechanical stress, or perhaps Rhopr-CRF/DH is part of a larger, more complex signalling pathway involving $\mathrm{AKH}$ and octopamine. With respect to reproduction, Rhopr-CRF/DH may be acting through similar pathways to delay reproductive processes until the blood meal is processed and nutrients are available to meet the high energy demands.

\section{Limitations (dsRNA)}

This work would have been strengthened had RNAi of the Rhopr-CRF/DH and Rhopr-CRF/DHR2B transcripts been reflected in reduced protein levels. By reducing expression of the peptide in the CNS, particularly, preventing Rhopr-CRF/DH release from the lateral NSCs of the MTGM, a more definitive conclusion would be made with respect to the involvement of these Rhopr$\mathrm{CRF} / \mathrm{DH}$ stores in feeding. It is expected that if Rhopr-CRF/DH release from the NSCs in the MTGM were disrupted, termination of feeding would not occur once a sufficient meal was taken or stress threshold reached. It is possible that without this termination signal, mechanical damage to the gut or abdomen would be observed, as the insect would continue feeding indefinitely. With respect to reproduction, in the absence of Rhopr-CRF/DH, it is expected that reproductive capacity would increase, perhaps through increased contraction of the oviducts. If Rhopr$\mathrm{CRF} / \mathrm{DH}$ indeed signals for the insect to delay oogenesis and other reproductive processes until nutrients from the blood meal are processed and available, its absence may result in the insect initiating these processes when the energy demands cannot be met. 
Knockdown of the receptor would serve a different but complementary purpose, by allowing investigation of the physiological and behavioural effects of disrupted Rhopr-CRF/DH signalling at various target tissues. The problem with this procedure is that Rhopr-CRF/DH-R2B is found throughout the $R$. prolixus system, and so its knockdown is likely to manifest in a multitude of phenotypes, but a mechanism specific to a phenotype would not be easily identified because injection of dsRNA into the haemolymph would have the potential to induce RNAi in all of the tissues producing the receptor.

\section{Summary}

In summary, this work supports the notion of Rhopr-CRF/DH as a multifunctional neurohormone in $R$. prolixus, acting not only as a diuretic, but also involved in the regulation of feeding and reproduction. Presence of Rhopr-CRF/DH in the haemolymph prior to feeding and after mating reduced size of meal taken, and the quantity of eggs produced and laid, respectively. Rhopr-CRF/DH also inhibited spontaneous contractions of the lateral oviducts in vivo in a dosedependent manner, highlighting this as a possible mechanism by which this neurohormone diminishes reproductive capacity. While the exact molecular and physiological mechanisms underlying these findings are not fully understood, based on what is known about this insect, together with findings in other insects, a model is proposed (Fig. 1). 
Figure 1. A schematic depicting the known (solid lines) and hypothesized (dotted lines) mechanisms underlying the behavioural effects of Rhopr-CRF/DH localized in NSCs in the brain (orange) and MTGM (green).

(1) Rhopr-CRF/DH is released from neurosecretory cells (NSCs) in the MTGM during feeding, and is known to act on the midgut and Malpighian tubules (MTs) to stimulate absorption and secretion, respectively; (2) Rhopr-CRF/DH, as it does in the locust, may also alter taste sensilla and thereby food perception by causing closure of pores on this sensory organ leading to cessation of feeding; (3) Rhopr-CRF/DH release from NSCs in the brain can, through binding its receptor on the ovaries, disrupt nutrient uptake and vitellogenesis, and (4) may lead to inhibition of the contractile activity of the lateral oviducts. The behavioural manifestations of these mechanisms of action by Rhopr-CRF/DH include diuresis, and reduced feeding and delayed reproductive competency.

Drawing of digestive system adapted from Zach McLaughlin and drawing of $R$. prolixus head adapted from images in: Escandón-Vargas, K., Muñoz C.A., Salazar, L., 2017. Blood-feeding of Rhodnius prolixus. Biomédica. 37. doi: 10.7705/biomedical.v34i2.3304. 
PHYSIOLOGICAL CONTROL

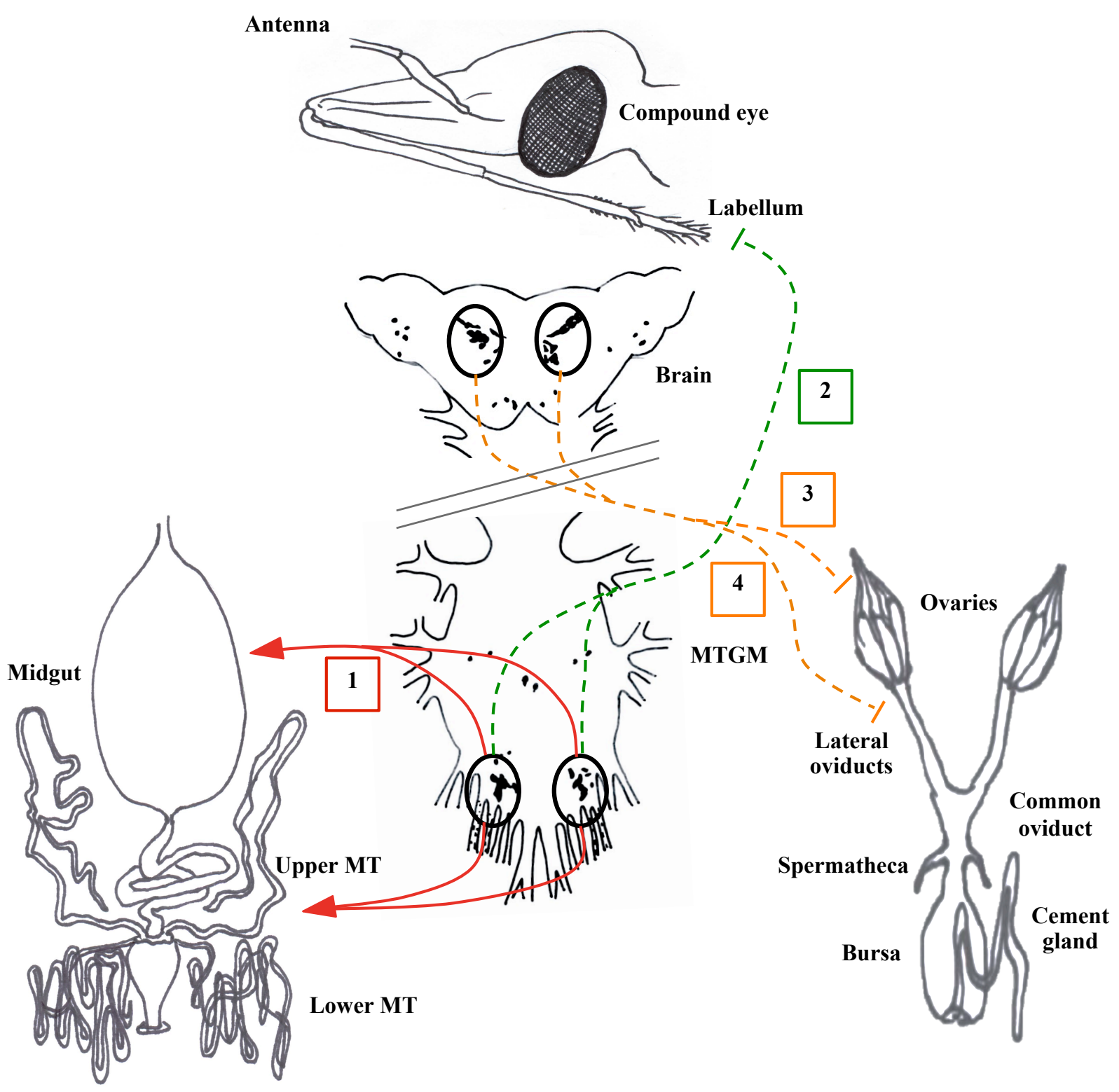

\section{BEHAVIOURS}

$\uparrow$ Diuresis via 1

$\downarrow \quad$ Feeding via 2 $\downarrow$ Oogenesis via 3

$\downarrow \quad$ Oviduct contractions via 4

$\downarrow$ Oviposition via $3 / 4$ 


\section{Future directions}

Future experiments will focus on optimization of the RNAi procedure to investigate the function of Rhopr-CRF/DH signalling in feeding and reproductive processes, through time course analysis of transcript and protein levels over two feeding bouts (see Chapter 3 Discussion). Western blot analysis to determine the levels of Rhopr-CRF/DH in the haemolymph at time points pre-, during, and post feeding and throughout the reproductive cycle would provide insight into critical times at which Rhopr-CRF/DH activity is highest. Also using Western blot analysis, haemolymph levels of known stress hormones, octopamine and AKH, can be tested at the same time points, to investigate the potential correlation between their activities and that of Rhopr-CRF/DH. Finally, identification and cloning of the other Rhopr-CRF/DH receptor (Lee et al., 2016), followed by spatial expression analysis, would allow investigation of potential differences in function compared to Rhopr-CRF/DH-R2B. 


\section{References}

Ampleford, E.J., Davey, K.G., 1989. Egg laying in the insect Rhodnius prolixus is timed in a circadian fashion. J. Insect Physiol. 35, 183-187. doi:10.1016/0022-1910(89)90003-6

Audsley, N., Goldsworthy, G.J., Coast, G.M., 1997. Circulating levels of Locusta diuretic hormone: The effect of feeding. Peptides 18, 59-65. doi:10.1016/S0196-9781(96)00234-3

Bernays, E.A., Mordue (Luntz), A.J., 1973. Changes in the palp tip sensilla of Locusta migratoria in relation to feeding: The effects of different levels of hormone. Comp. Biochem. Physiol. -- Part A Physiol. 45, 451-454. doi:10.1016/0300-9629(73)90452-0

Chiang, R.G., Davey, K.G., 1988. A Novel Receptor Capable of Monitoring Applied Pressure in the Abdomen of an Insect. Science (80-. ). 241, 1665-1667. doi:10.1126/science.241.4873.1665

Choi, J., Smitz, J., 2014. Luteinizing hormone and human chorionic gonadotropin: Origins of difference. Mol. Cell. Endocrinol. doi:10.1016/j.mce.2013.12.009

Davenport, A.P., Evans, P.D., 1984. Stress-induced changes in the octopamine levels of insect haemolymph. Insect Biochem. 14, 135-143. doi:10.1016/0020-1790(84)90021-0

Davey, K.G., 1993. Hormonal integration of egg production in Rhodnius prolixus. Integr. Comp. Biol. 33, 397-402. doi:10.1093/icb/33.3.397

Davey, K.G., 1981. Hormonal control of vitellogenin uptake in rhodnius prolixus stål. Integr. Comp. Biol. 21, 701-705. doi:10.1093/icb/21.3.701

Even, N., Devaud, J.-M., Barron, A.B., 2012. General Stress Responses in the Honey Bee. Insects 3, 1271-1298. doi:10.3390/insects3041271

Gäde, G., Goldsworthy, G.J., 2003. Insect peptide hormones: A selective review of their physiology and potential application for pest control. Pest Manag. Sci. doi:10.1002/ps.755

Gáliková, M., Diesner, M., Klepsatel, P., Hehlert, P., Xu, Y., Bickmeyer, I., Predel, R., Kühnlein, R.P., 2015. Energy homeostasis control in drosophila adipokinetic hormone mutants. Genetics 201, 665-683. doi:10.1534/genetics.115.178897

Heiman, M.L., Ahima, R.S., Craft, L.S., Schoner, B., Stephens, T.W., Flier, J.S., 1997. Leptin inhibition of the hypothalamic-pituitary-adrenal axis in response to stress. Endocrinology 138, 3859-3863. doi:10.1210/en.138.9.3859

Kinsey-Jones, J.S., Li, X.F., Knox, A.M.I., Lin, Y.S., Milligan, S.R., Lightman, S.L., O’Byrne, K.T., 2010. Corticotrophin-releasing factor alters the timing of puberty in the female rat. J. Neuroendocrinol. 22, 102-109. doi:10.1111/j.1365-2826.2009.01940.x

Kovács, K.J., 2013. CRH: The link between hormonal-, metabolic- and behavioral responses to stress. J. Chem. Neuroanat. 54, 25-33. doi:10.1016/j.jchemneu.2013.05.003 
Lee, H.R., Zandawala, M., Lange, A.B., Orchard, I., 2016. Isolation and characterization of the corticotropin-releasing factor-related diuretic hormone receptor in Rhodnius prolixus. Cell. Signal. 28, 1152-1162. doi:10.1016/j.cellsig.2016.05.020

Li, F.X., Bowe, J.E., Lightman, S.L., O’Byrne, K.T., 2005. Role of corticotropin-releasing factor receptor-2 in stress-induced suppression of pulsatile luteinizing hormone secretion in the rat. Endocrinology 146, 318-322. doi:10.1210/en.2004-0950

Mollayeva, S., Orchard, I., Lange, A.B., 2017. The involvement of Rhopr-CRF/DH in feeding and reproduction in the blood-gorging insect Rhodnius prolixus. Gen. Comp. Endocrinol. doi: 10.196/j.ygcen.2017.07.005 (in press)

Rivier, C., Vale, W., 1984. Influence of corticotropin-releasing factor on reproductive functions in the rat. Endocrinology 114, 914-921. doi:10.1210/endo-114-3-914

Simpson, S.J., Bernays, E.A., 1983. The regulation of feeding: locusts and blowflies are not so different from mammals. Appetite 4, 313-46. doi:10.1016/S0195-6663(83)80024-5

Stengel, A., Taché, Y., 2014. CRF and urocortin peptides as modulators of energy balance and feeding behavior during stress. Front. Neurosci. doi:10.3389/fnins.2014.00052

Sun, Y., Hunt, S., Sah, P., 2015. Norepinephrine and Corticotropin-Releasing Hormone: Partners in the Neural Circuits that Underpin Stress and Anxiety. Neuron.

doi:10.1016/j.neuron.2015.07.022

Te Brugge, V., Ianowski, J.P., Orchard, I., 2009. Biological activity of diuretic factors on the anterior midgut of the blood-feeding bug, Rhodnius prolixus. Gen. Comp. Endocrinol. 162, 105112. doi:10.1016/j.ygcen.2009.01.025

van Wielendaele, P., Dillen, S., Marchal, E., Badisco, L., Vanden Broeck, J., 2012. CRF-like diuretic hormone negatively affects both feeding and reproduction in the desert locust, Schistocerca gregaria. PLoS One 7. doi:10.1371/journal.pone.0031425

Yakabi, K., Noguchi, M., Ohno, S., Ro, S., Onouchi, T., Ochiai, M., Takabayashi, H., Takayama, K., Harada, Y., Sadakane, C., Hattori, T., 2011. Urocortin 1 reduces food intake and ghrelin secretion via CRF(2) receptors. Am. J. Physiol. Endocrinol. Metab. 301, E72-82. doi:10.1152/ajpendo.00695.2010 